\title{
THE TROPICAL VERTEX
}

\author{
M. GROSS, R. PANDHARIPANDE, AND B. SIEBERT
}

\begin{abstract}
Elements of the tropical vertex group are formal families of symplectomorphisms of the 2-dimensional algebraic torus. We prove ordered product factorizations in the tropical vertex group are equivalent to calculations of certain genus 0 relative Gromov-Witten invariants of toric surfaces. The relative invariants which arise have full tangency to a toric divisor at a single unspecified point. The method uses scattering diagrams, tropical curve counts, degeneration formulas, and exact multiple cover calculations in orbifold Gromov-Witten theory.
\end{abstract}

\section{Contents}

Introduction

0.1. Automorphisms of the torus

0.2. Tropical vertex group

0.3. Commutators

0.4. Toric surfaces

0.5. Formula

0.6. Plan of the paper

0.7. Acknowledgments

1. Scattering

1.1. Tropical vertex group (again)

1.2. Scattering diagrams

1.3. Path ordered products

1.4. Asymptotic diagrams and factorization

2. Tropical curves

2.1. Definitions

2.2. Scattering diagrams 20

2.3. Tropical counts 23

3. From tropical to holomorphic counts 26

3.1. Holomorphic counts 26

3.2. Equivalence 29

3.3. Degenerate case

Date: February 4th, 2009. 
4. Gromov-Witten theory 30

4.1. Overview 30

4.2. Desingularization 31

4.3. Stable relative maps 32

4.4. Gromov-Witten theory for $X^{\circ}$

5. Formulas in the tropical vertex group

5.1. Simple blow-ups 38

5.2. Gromov-Witten theory 39

5.3. Degeneration $\quad 39$

5.4. First commutator formulas $\quad 42$

5.5. Orbifold blow-ups $\quad 44$

5.6. Full commutator formulas

5.7. Ordered product formulas 49

5.8. Higher genus $\quad 49$

6. BPS state counts 50

6.1. Log Calabi-Yau 50

6.2. Multiple cover contributions 50

6.3. Conjectures $\quad 52$

6.4. Examples 53

Appendix: Tropical/holomorphic counts 55

References

INTRODUCTION

0.1. Automorphisms of the torus. The 2-dimensional complex torus has very few automorphisms

$$
\theta: \mathbb{C}^{*} \times \mathbb{C}^{*} \rightarrow \mathbb{C}^{*} \times \mathbb{C}^{*}
$$

as an algebraic group. Since $\theta$ must take each component $\mathbb{C}^{*}$ to a 1 -dimensional subtorus,

$$
\operatorname{Aut}_{\mathbb{C}}^{\mathrm{Gr}}\left(\mathbb{C}^{*} \times \mathbb{C}^{*}\right) \stackrel{\cong}{=} \mathrm{GL}_{2}(\mathbb{Z}) .
$$


As a complex algebraic variety, $\mathbb{C}^{*} \times \mathbb{C}^{*}$ has, in addition, only the automorphisms obtained by the translation action on itself 1

$$
1 \rightarrow \mathbb{C}^{*} \times \mathbb{C}^{*} \rightarrow \operatorname{Aut}_{\mathbb{C}}\left(\mathbb{C}^{*} \times \mathbb{C}^{*}\right) \rightarrow \operatorname{Aut}_{\mathbb{C}}^{\mathrm{Gr}}\left(\mathbb{C}^{*} \times \mathbb{C}^{*}\right) \rightarrow 1
$$

A much richer algebraic structure appears if formal 1-parameter families of automorphisms of $\mathbb{C}^{*} \times \mathbb{C}^{*}$ are considered,

$$
A=\operatorname{Aut}_{\mathbb{C}[t]]}\left(\mathbb{C}^{*} \times \mathbb{C}^{*} \times \operatorname{Spec}(\mathbb{C}[[t]])\right) .
$$

Let $x$ and $y$ be the coordinates of the two factors of $\mathbb{C}^{*} \times \mathbb{C}^{*}$. Then,

$$
\mathbb{C}^{*} \times \mathbb{C}^{*}=\operatorname{Spec}\left(\mathbb{C}\left[x, x^{-1}, y, y^{-1}\right]\right) .
$$

We may alternatively view $A$ as a group of algebra automorphisms,

$$
A=\operatorname{Aut}_{\mathbb{C}[t]]]}\left(\mathbb{C}\left[x, x^{-1}, y, y^{-1}\right][[t]]\right) .
$$

Nontrivial elements of $A$ are easily found. Let $(a, b) \in \mathbb{Z}^{2}$ be a nonzero vector, and let $f \in \mathbb{C}\left[x, x^{-1}, y, y^{-1}\right][[t]]$ be a function of the form

$$
f=1+t x^{a} y^{b} \cdot g\left(x^{a} y^{b}, t\right), \quad g(z, t) \in \mathbb{C}[z][[t]] .
$$

We specify the values of an automorphism on $x$ and $y$ by

$$
\theta_{(a, b), f}(x)=f^{-b} \cdot x, \quad \theta_{(a, b), f}(y)=f^{a} \cdot y .
$$

The assignment (0.1) extends uniquely to determine an element $\theta_{(a, b), f} \in A$ with

$$
\theta_{(a, b), f}^{-1}=\theta_{(a, b), f^{-1}} .
$$

0.2. Tropical vertex group. The tropical vertex group $H \subseteq A$ is the completion with respect to the maximal ideal $(t) \subseteq \mathbb{C}[[t]]$ of the subgroup generated by all elements of the form $\theta_{(a, b), f}$. A more natural characterization of $H$ via the associated Lie algebra is reviewed in Section 1.1.

The torus $\mathbb{C}^{*} \times \mathbb{C}^{*}$ has a standard holomorphic symplectic form given by

$$
\omega=\frac{d x}{x} \wedge \frac{d y}{y} .
$$

Let $S \subseteq A$ be the subgroup of automorphisms preserving $\omega$,

$$
S=\left\{\theta \in A \mid \theta^{*}(\omega)=\omega\right\} .
$$

A direct calculation shows $H \subseteq S$.

\footnotetext{
${ }^{1}$ We leave the elementary proof to the reader. An argument can be found by using the characterization

$$
\phi(z)=\lambda \cdot z^{k} \quad \lambda \in \mathbb{C}^{*}, k \in \mathbb{Z}
$$

of all algebraic maps $\phi: \mathbb{C}^{*} \rightarrow \mathbb{C}^{*}$.
} 
A slight variant of the tropical vertex group $H$ first arose in the study of affine structures by Kontsevich and Soibelman in [13. Further development, related to mirror symmetry and tropical geometry, can be found in [10]. Recently, the tropical vertex group has played a role in wall-crossing formulas for counting invariants in derived categories [14].

0.3. Commutators. The first question we can ask about the tropical vertex group is to find a formula for the commutators of the generators. The answer, the main result of the paper, turns out to be surprisingly subtle. The commutator formula is expressed in terms of the relative Gromov-Witten theories of toric surfaces.

Perhaps the simplest nontrivial case to consider is the commutator of the elements

$$
S_{\ell_{1}}=\theta_{(1,0),(1+t x)^{\ell_{1}}} \text { and } T_{\ell_{2}}=\theta_{(0,1),(1+t y)^{\ell_{2}}}
$$

where $\ell_{1}, \ell_{2}>0$. By an elementary result of [13] reviewed in Section [1.3, there exists a unique factorization

$$
T_{\ell_{2}}^{-1} \circ S_{\ell_{1}} \circ T_{\ell_{2}} \circ S_{\ell_{1}}^{-1}=\prod^{\rightarrow} \theta_{(a, b), f_{(a, b)}}
$$

where the product on the right is over all primitive vectors $(a, b) \in \mathbb{Z}^{2}$ lying strictly in the first quadrant 2 The order is determined by increasing slopes of the vectors $(a, b)$. The question is what are the functions $f_{(a, b)}$ associated to the slopes?

0.4. Toric surfaces. Let $(a, b) \in \mathbb{Z}^{2}$ be a primitive vector lying strictly in the first quadrant. The rays generated by $(-1,0),(0,-1)$, and $(a, b)$ determine a complete rational fan in $\mathbb{R}^{2}$. Let $X_{a, b}$ be the associated toric surface 3 with toric divisors

$$
D_{1}, D_{2}, D_{\text {out }} \subseteq X_{a, b}
$$

corresponding to the respective rays. Let

$$
X_{a, b}^{o} \subseteq X_{a, b}
$$

be the open surface obtained by removing the three toric fixed points. Let $D_{1}^{o}, D_{2}^{o}, D_{\text {out }}^{o}$ be the restrictions of the toric divisors to $X_{a, b}^{o}$.

We denote ordered partitions $\mathbf{Q}$ of length $\ell$ by $q_{1}+\ldots+q_{\ell}$. Ordered partitions differ from usual partitions in two basic ways. First, the ordering of the parts matters. Second, the parts $q_{i}$ are required only to be non-negative integers ( 0 is permitted). The size $|\mathbf{Q}|$ is the sum of the parts.

\footnotetext{
${ }^{2} \mathrm{~A}$ vector $(a, b)$ is primitive if it is not divisible in $\mathbb{Z}^{2}$. Primitivity implies $(a, b) \neq(0,0)$. Strict inclusion in the first quadrant is equivalent to $a>0$ and $b>0$.

${ }^{3} X_{a, b}$ is simply a weighted projective plane. Arbitrary toric surfaces will arise in the study of more general commutators.
} 
Let $k \geq 1$. Let $\mathbf{P}_{a}=p_{1}+\ldots+p_{\ell_{a}}$ and $\mathbf{P}_{b}=p_{1}^{\prime}+\ldots+p_{\ell_{b}}^{\prime}$ be ordered partitions of size $a k$ and $b k$ respectively. Denote the pair by $\mathbf{P}=\left(\mathbf{P}_{a}, \mathbf{P}_{b}\right)$. Let

$$
\nu: X_{a, b}[\mathbf{P}] \rightarrow X_{a, b}
$$

be the blow-up of $X_{a, b}$ along $\ell_{a}$ and $\ell_{b}$ distinct points of $D_{1}^{o}$ and $D_{2}^{o}$. Let

$$
X_{a, b}^{o}[\mathbf{P}]=\nu^{-1}\left(X_{a, b}^{o}\right) .
$$

Let $\beta_{k} \in H_{2}\left(X_{a, b}, \mathbb{Z}\right)$ be the unique class with intersection numbers

$$
\beta_{k} \cdot D_{1}=a k, \quad \beta_{k} \cdot D_{2}=b k, \quad \beta_{k} \cdot D_{\text {out }}=k .
$$

Let $E_{i}$ and $E_{j}^{\prime}$ be the $i^{\text {th }}$ and $j^{\text {th }}$ exceptional divisors over $D_{1}^{o}$ and $D_{2}^{o}$. Let

$$
\beta_{k}[\mathbf{P}]=\nu^{*}\left(\beta_{k}\right)-\sum_{i=1}^{\ell_{a}} p_{i}\left[E_{i}\right]-\sum_{j=1}^{\ell_{b}} p_{j}^{\prime}\left[E_{j}^{\prime}\right] \in H_{2}\left(X_{a, b}[\mathbf{P}], \mathbb{Z}\right) .
$$

By a parameter count, the moduli space $\overline{\mathfrak{M}}\left(X_{a, b}^{o}[\mathbf{P}] / D_{\text {out }}^{o}\right)$ of genus 0 maps to $X^{o}[\mathbf{P}]$ in class $\beta_{k}[\mathbf{P}]$ with full contact order $k$ at an unspecified point of $D_{\text {out }}^{o}$ is of virtual dimension 0. In Section 4, we will show the corresponding Gromov-Witten invariant

$$
N_{a, b}[\mathbf{P}] \in \mathbb{Q}
$$

is well-defined 4

0.5. Formula. Since the series $f_{(a, b)}$ in (0.2) starts with 1, we may take the logarithm. Homogeneity constraints determine the behavior of the variable $t$. We define the coefficients $c_{a, b}^{k}\left(\ell_{1}, \ell_{2}\right) \in \mathbb{Q}$ by

$$
\log f_{(a, b)}=\sum_{k \geq 1} k c_{a, b}^{k}\left(\ell_{1}, \ell_{2}\right) \cdot(t x)^{a k}(t y)^{b k} .
$$

The function $f_{(a, b)}$ is linked to Gromov-Witten theory by the following result.

Theorem 0.1. We have

$$
c_{a, b}^{k}\left(\ell_{1}, \ell_{2}\right)=\sum_{\left|\mathbf{P}_{a}\right|=a k} \sum_{\left|\mathbf{P}_{b}\right|=b k} N_{a, b}\left[\left(\mathbf{P}_{a}, \mathbf{P}_{b}\right)\right]
$$

where the sums are over all ordered partitions $\mathbf{P}_{a}$ of size ak and length $\ell_{1}$ and $\mathbf{P}_{b}$ of size bk and length $\ell_{2}$.

\footnotetext{
${ }^{4}$ The only issue to understand is the relative Gromov-Witten theory of the open geometry $X_{a, b}^{o}[\mathbf{P}] / D_{\text {out }}^{o}$. We will show there is sufficient properness here to define Gromov-Witten invariants in the usual way.
} 
Theorem 0.1 is the first in a series of results. A refinement of Theorem 0.1 proven in Section 5.4 shows the invariants $N_{a, b}[\mathbf{P}]$ are determined by ordered product factorizations of commutators in the tropical vertex group. In fact, the tropical vertex group calculations are much simpler (and much more conceptually appealing) than the alternative methods available for calculating $N_{a, b}[\mathbf{P}]$.

Commutator formulas for arbitrary generators $\theta_{(a, b), f}$ and $\theta_{\left(a^{\prime}, b^{\prime}\right), f^{\prime}}$ of the tropical vertex group are proven in Section 5. For general functions $f$ and $f^{\prime}$, orbifold blowups of toric surfaces replace the ordinary blow-ups discussed above. As a result, the relations between generators of the tropical vertex group are completely described in terms of Gromov-Witten invariants.

The tropical vertex group was used in [13] to construct rigid analytic K3 surfaces from affine 2-spheres with singularities. The group was similarly used in [10] to construct explicit degenerations of Calabi-Yau manifolds from integral affine manifolds with singularities. The latter work gives a precise description of the B-model for Calabi-Yau manifolds (as well as manifolds with effective anti-canonical bundle). The commutator formula of Theorem 0.1 provides an interpretation of the tropical vertex group in terms of curve counts on the A-model side. Hence, Theorem 0.1 should be viewed as a mirror symmetry relation.

Special cases of commutators in the tropical vertex group have direct interpretation in the wall-crossing work of [14, 23]. The

$$
\ell=\ell_{1}=\ell_{2}
$$

case of Theorem 0.1 is related to the quiver $Q_{\ell}$ with two vertices and $\ell$ arrows (in the same direction). Reineke [23] has proven the functions $f_{(a, b)}$ are then determined by the Euler characteristics of the moduli spaces of semistable representations of $Q_{\ell}$ with dimension vectors along the ray generated by $(a, b)$. Whether such alternative interpretations hold for more general commutators is an interesting question.

0.6. Plan of the paper. We start with a formal discussion of the tropical vertex group and the associated scattering diagrams for path oriented products in Section 1 . The scattering diagram expansions connect the commutators to tropical curve counts explained in Section 2, The tropical counts are related to the enumeration of holomorphic curves in Section 3 following [19, 20]. The Gromov-Witten theory of open toric surfaces is discussed in Section 4. The commutator formulas are proven in Section 5 via degeneration and exact Gromov-Witten calculations.

The Gromov-Witten invariants $N_{a, b}[\mathbf{P}]$ which arise in Theorem 0.1 are virtual counts with complicated multiple cover contributions of excess dimension. The corresponding (conjectural) BPS structure is discussed at the end of the paper in Section 6. The 
method also applies to neighboring questions such as the enumeration of degree $d$ rational curves in $\mathbb{P}^{2}$ with full tangency at a single point to a nonsingular elliptic curve $E \subseteq \mathbb{P}^{2}$ studied in [5, 25.

0.7. Acknowledgments. We thank J. Bryan, T. Graber, J. de Jong, J. Kollár, M. Kontsevich, D. Maulik, Y. Soibelman, and N. Takahashi for many related comments and discussions. M. G. was partially supported by NSF grant DMS-0805328 and the DFG. R. P. was partially supported by NSF grant DMS-0500187 and the Clay foundation.

\section{Scattering}

1.1. Tropical vertex group (again). We begin by reviewing ideas from 10. While most of the material we need can be extracted from [10], the development there is in much greater generality than we need, and a number of points simplify in our context. We will give a completely self-contained exposition.

We fix once and for all a lattice $M \cong \mathbb{Z}^{2}$ with basis $e_{1}=(1,0)$ and $e_{2}=(0,1)$. Let

$$
N=\operatorname{Hom}_{\mathbb{Z}}(M, \mathbb{Z}), \quad M_{\mathbb{R}}=M \otimes_{\mathbb{Z}} \mathbb{R}, \quad N_{\mathbb{R}}=N \otimes_{\mathbb{Z}} \mathbb{R} .
$$

For $m \in M$, let $z^{m} \in \mathbb{C}[M]$ denote the corresponding element in the group ring. Let

$$
x=z^{e_{1}}, \quad y=z^{e_{2}} .
$$

Then, $\mathbb{C}[M]$ is simply the ring of Laurent polynomials in $x$ and $y$.

In what follows, let $R$ be an Artin local $\mathbb{C}$-algebra or a complete local $\mathbb{C}$-algebra. 5 Let $\mathfrak{m}_{R} \subseteq R$ be the maximal ideal. We define the module of log derivations of

$$
\mathbb{C}[M] \widehat{\otimes}_{\mathbb{C}} R=\lim _{\longleftarrow} \mathbb{C}[M] \otimes_{\mathbb{C}} R / \mathfrak{m}_{R}^{k}
$$

to be

$$
\Theta\left(\mathbb{C}[M] \widehat{\otimes}_{\mathbb{C}} R\right)=\operatorname{Hom}\left(M, \mathbb{C}[M] \widehat{\otimes}_{\mathbb{C}} R\right)=\left(\mathbb{C}[M] \widehat{\otimes}_{\mathbb{C}} R\right) \otimes_{\mathbb{Z}} N .
$$

A $\log$ derivation $\xi$ induces an ordinary derivation $\bar{\xi}$ of $\mathbb{C}[M] \widehat{\otimes}_{\mathbb{C}} R$ over $R$ via the formula

$$
\bar{\xi}\left(z^{m}\right)=\xi(m) z^{m} .
$$

We will write $a \partial_{n}$ for $a \otimes n \in\left(\mathbb{C}[M] \widehat{\otimes}_{\mathbb{C}} R\right) \otimes_{\mathbb{Z}} N=\Theta\left(\mathbb{C}[M] \widehat{\otimes}_{\mathbb{C}} R\right)$ and for the associated ordinary derivation,

$$
\left(a \partial_{n}\right)\left(z^{m}\right)=a\langle m, n\rangle z^{m} .
$$

Let $\mathfrak{g}_{R}=\mathfrak{m}_{R} \Theta\left(\mathbb{C}[M] \widehat{\otimes}_{\mathbb{C}} R\right)$. Given any $\xi \in \mathfrak{g}_{R}$, we obtain an element

$$
\exp (\xi) \in \operatorname{Aut}_{R}\left(\mathbb{C}[M] \widehat{\otimes}_{\mathbb{C}} R\right)
$$

\footnotetext{
${ }^{5}$ In the introduction, we took $R=\mathbb{C}[[t]]$.
} 
of the group of ring automorphisms defined by

$$
\exp (\xi)(a)=\operatorname{Id}(a)+\sum_{i=1}^{\infty} \frac{\bar{\xi}^{i}(a)}{i !} .
$$

The series makes sense by the assumptions on $R$.

In fact, $\mathfrak{g}_{R}$ is a Lie algebra under the natural bracket defined by

$$
\begin{aligned}
{\left[z^{m} \partial_{n}, z^{m^{\prime}} \partial_{n^{\prime}}\right] } & =\left(z^{m} \partial_{n}\left(z^{m^{\prime}}\right)\right) \partial_{n^{\prime}}-\left(z^{m^{\prime}} \partial_{n^{\prime}}\left(z^{m}\right)\right) \partial_{n} \\
& =z^{m+m^{\prime}}\left(\left\langle m^{\prime}, n\right\rangle \partial_{n^{\prime}}-\left\langle m, n^{\prime}\right\rangle \partial_{n}\right) \\
& =z^{m+m^{\prime}} \partial_{\left\langle m^{\prime}, n\right\rangle n^{\prime}-\left\langle m, n^{\prime}\right\rangle n} .
\end{aligned}
$$

By the Baker-Campbell-Hausdorff formula, the subset

$$
G_{R}=\left\{\exp (\xi) \mid \xi \in \mathfrak{g}_{R}\right\}
$$

of $\operatorname{Aut}_{R}\left(\mathbb{C}[M] \widehat{\otimes}_{\mathbb{C}} R\right)$ is a subgroup.

The subspace $\mathfrak{h}_{R} \subseteq \mathfrak{g}_{R}$ defined by

$$
\mathfrak{h}_{R}=\bigoplus_{m \in M \backslash\{0\}} z^{m}\left(\mathfrak{m}_{R} \otimes m^{\perp}\right)
$$

is closed under the Lie bracket, and hence via exponentiation defines a subgroup

$$
\mathbb{V}_{R} \subseteq G_{R}
$$

which we call the tropical vertex group. The subgroup $\mathbb{V}_{R}$ is closed in the $\mathfrak{m}_{R}$-adic topology.

1.2. Scattering diagrams. We will depict certain products of automorphisms of the form $\theta_{(a, b), f}$ as introduced in the Introduction by diagrams in the plane $M_{\mathbb{R}}$. Automorphisms of the form $\theta_{(a, b), f}$ in fact generate $\mathbb{V}_{R}$, and this will give us a way of describing elements in $\mathbb{V}_{R}$.

Definition 1.1. A ray or line is a pair $\left(\mathfrak{d}, f_{\mathfrak{d}}\right)$ such that

- $\mathfrak{d} \subseteq M_{\mathbb{R}}$ is given by

$$
\mathfrak{d}=m_{0}^{\prime}+\mathbb{R}_{\geq 0} m_{0}
$$

if $\mathfrak{d}$ is a ray and

$$
\mathfrak{d}=m_{0}^{\prime}+\mathbb{R} m_{0}
$$

if $\mathfrak{d}$ is a line, for some $m_{0}^{\prime} \in M_{\mathbb{R}}$ and $m_{0} \in M \backslash\{0\}$. The set $\mathfrak{d}$ is called the support of the line or ray. If $\mathfrak{d}$ is a ray, $m_{0}^{\prime}$ is called the initial point of the ray, written as $\operatorname{Init}(\mathfrak{d})$.

- $f_{\mathfrak{o}} \in \mathbb{C}\left[z^{m_{0}}\right] \widehat{\otimes}_{\mathbb{C}} R \subseteq \mathbb{C}[M] \widehat{\otimes}_{\mathbb{C}} R$.

- $f_{\mathfrak{o}} \equiv 1 \bmod z^{m_{0}} \mathfrak{m}_{R}$. 
Definition 1.2. A scattering diagram $\mathfrak{D}$ is a set of lines and rays such that for every power $k>0$, there are only a finite number of $\left(\mathfrak{d}, f_{\mathfrak{d}}\right) \in \mathfrak{D}$ with $f_{\mathfrak{d}} \not \equiv 1 \bmod \mathfrak{m}_{R}^{k}$.

If $\mathfrak{D}$ is a scattering diagram, we write

$$
\operatorname{Sing}(\mathfrak{D})=\bigcup_{\mathfrak{d} \in \mathfrak{D}} \partial \mathfrak{d} \cup \bigcup_{\substack{\mathfrak{o}_{1}, \mathfrak{d}_{2} \\ \operatorname{dim} \mathfrak{d}_{1} \cap \mathfrak{d}_{2}=0}} \mathfrak{d}_{1} \cap \mathfrak{d}_{2} .
$$

Here $\partial \mathfrak{d}=\{\operatorname{Init}(\mathfrak{d})\}$ if $\mathfrak{d}$ is a ray, and is empty if $\mathfrak{d}$ is a line.

We are using a different sign convention than in [10]. Here, we view a monomial $z^{m}$ as propagating in the direction $m$, while in [10, monomials propagate in the direction $-m$. The negative sign is necessary in [10]. We do not need the sign for our purposes here, so we dispense with it.

1.3. Path ordered products. Consider a smooth immersion

$$
\gamma:[0,1] \rightarrow M_{\mathbb{R}} \backslash \operatorname{Sing}(\mathfrak{D})
$$

with endpoints not contained in the scattering diagram $\mathfrak{D}$. If all intersections of $\gamma$ with the rays and lines of $\mathfrak{D}$ are transverse, we can define $\theta_{\gamma, \mathfrak{D}} \in \mathbb{V}_{R}$, the $\gamma$-ordered product of $\mathfrak{D}$ as follows.

For each power $k>0$, we can find numbers

$$
0<t_{1} \leq t_{2} \leq \cdots \leq t_{s}<1
$$

and elements $\mathfrak{d}_{i} \in \mathfrak{D}$ with $f_{\mathfrak{d}_{i}} \not \equiv 1 \bmod \mathfrak{m}_{R}^{k}$ such that

$$
\gamma\left(t_{i}\right) \in \mathfrak{d}_{i},
$$

$\mathfrak{d}_{i} \neq \mathfrak{d}_{j}$ if $t_{i}=t_{j}$ and $i \neq j$, and $s$ taken as large as possible. For each $i$, define $\theta_{\mathfrak{d}_{i}}$ to be

$$
\theta_{\mathfrak{D}_{i}}=\exp \left(\log \left(f_{\mathfrak{o}_{i}}\right) \partial_{n_{0}}\right)
$$

where $n_{0} \in N$ is primitive, annihilates the tangent space to $\mathfrak{d}_{i}$, and is uniquely determined by the sign convention

$$
\left\langle n_{0}, \gamma^{\prime}\left(t_{i}\right)\right\rangle>0
$$

We define

$$
\theta_{\gamma, \mathfrak{D}}^{k}=\theta_{\mathfrak{d}_{s}} \circ \cdots \circ \theta_{\mathfrak{d}_{1}}
$$

If $t_{i}=t_{i+1}$, then $\gamma\left(t_{i}\right) \in \mathfrak{d}_{i} \cap \mathfrak{d}_{i+1}$. Hence, $\operatorname{dim} \mathfrak{d}_{i} \cap \mathfrak{d}_{i+1}=1$. The elements $\theta_{\mathfrak{d}_{i}}$ and $\theta_{\mathfrak{d}_{i+1}}$ are easily seen to commute. Therefore, the product does not depend on any choices. Let

$$
\theta_{\gamma, \mathfrak{D}}=\lim _{k \rightarrow \infty} \theta_{\gamma, \mathfrak{D}}^{k}
$$




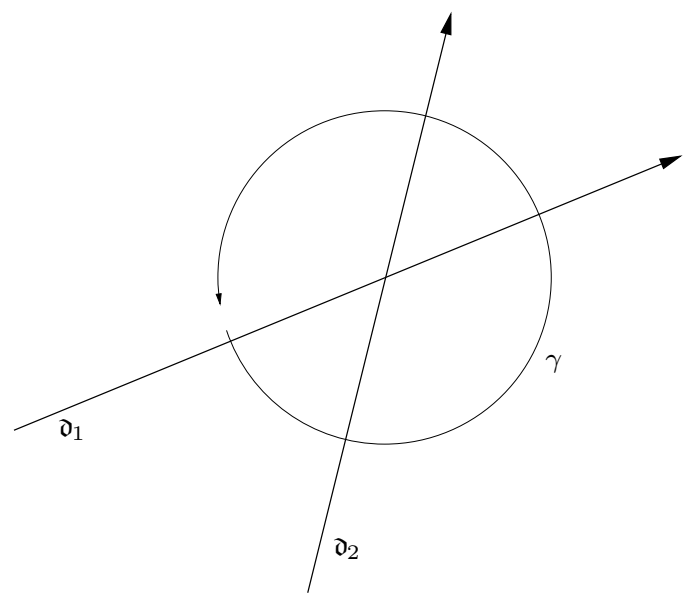

FIGURE 1.1.

Example 1.3. A basic example for us is given by the scattering diagram

$$
\mathfrak{D}=\left\{\left(\mathfrak{d}_{1}, f_{1}\right),\left(\mathfrak{d}_{2}, f_{2}\right)\right\}
$$

where $\mathfrak{d}_{1}, \mathfrak{d}_{2}$ are transverse lines through the origin. A suitably chosen loop $\gamma$ around the origin produces a commutator:

$$
\theta_{\gamma, \mathfrak{D}}=\theta_{2}^{-1} \theta_{1}^{-1} \theta_{2} \theta_{1}
$$

where the elements $\theta_{1}$ and $\theta_{2}$ are obtained from $\mathfrak{d}_{1}$ and $\mathfrak{d}_{2}$ respectively, see Figure 1.1 .

The following result was obtained by Kontsevich and Soibelman in [13] with a number of variants proved in [10]. Since the proof is so simple, we reproduce it here.

Theorem 1.4. Let $\mathfrak{D}$ be a scattering diagram. Then, there exists a scattering diagram $\mathrm{S}(\mathfrak{D})$ containing $\mathfrak{D}$ such that $\mathrm{S}(\mathfrak{D}) \backslash \mathfrak{D}$ consists only of rays, and such that $\theta_{\gamma, \mathrm{S}(\mathfrak{D})}=\mathrm{Id}$ for any closed loop $\gamma$ for which $\theta_{\gamma, \mathrm{S}(\mathfrak{D})}$ is defined.

Proof. We proceed inductively on $k$, showing that there exists a $\mathfrak{D}_{k}$ such that

$$
\theta_{\gamma, \mathfrak{D}_{k}} \equiv \mathrm{Id} \quad \bmod \mathfrak{m}_{R}^{k+1}
$$

for all closed loops $\gamma$ for which $\theta_{\gamma, \mathfrak{D}_{k}}$ is defined. We take $\mathfrak{D}_{0}=\mathfrak{D}$. To obtain $\mathfrak{D}_{k}$ from $\mathfrak{D}_{k-1}$, we proceed as follows. Let $\mathfrak{D}_{k-1}^{\prime}$ consist of those rays and lines $\mathfrak{d}$ in $\mathfrak{D}_{k-1}$ with $f_{\mathfrak{o}} \not \equiv 1 \bmod \mathfrak{m}_{R}^{k+1}$. This is a finite set, $\operatorname{so} \operatorname{Sing}\left(\mathfrak{D}_{k-1}^{\prime}\right)$ is finite also. Let $p \in \operatorname{Sing}\left(\mathfrak{D}_{k-1}^{\prime}\right)$. Let $\gamma_{p}$ be a closed simple loop around $p$, small enough so it contains no other points of $\operatorname{Sing}\left(\mathfrak{D}_{k-1}^{\prime}\right)$. Certainly,

$$
\theta_{\gamma_{p}, \mathfrak{D}_{k-1}} \equiv \theta_{\gamma_{p}, \mathfrak{D}_{k-1}^{\prime}} \quad \bmod \mathfrak{m}_{R}^{k+1}
$$


By the inductive assumption we can write uniquely

$$
\theta_{\gamma_{p}, \mathfrak{D}_{k-1}^{\prime}}=\exp \left(\sum_{i=1}^{s} c_{i} z^{m_{i}} \partial_{n_{i}}\right)
$$

with $m_{i} \in M \backslash\{0\}, n_{i} \in m_{i}^{\perp}$ primitive and $c_{i} \in \mathfrak{m}_{R}^{k}$. Let

$$
\mathfrak{D}[p]=\left\{\left(p+\mathbb{R}_{\geq 0} m_{i}, 1 \pm c_{i} z^{m_{i}}\right) \mid i=1, \ldots, s\right\} .
$$

The sign is chosen in each ray so that its contribution to $\theta_{\gamma_{p}, \mathfrak{D}[p]}$ is $\exp \left(-c_{i} z^{m_{i}} \partial_{n_{i}}\right)$ modulo $\mathfrak{m}_{R}^{k+1}$. Since $c_{i} z^{m_{i}} \partial_{n_{i}}$ is in the center of $\mathfrak{h}_{R / \mathfrak{m}_{R}^{k+1}}$, we see

$$
\theta_{\gamma_{p}, \mathfrak{D}_{k-1} \cup \mathfrak{D}[p]}=\mathrm{Id} \quad \bmod \mathfrak{m}_{R}^{k+1} .
$$

Similarly, any automorphism coming from $\mathfrak{D}[p]$ commutes with any automorphism coming from $\mathfrak{D}_{k-1}$ modulo $\mathfrak{m}_{R}^{k+1}$, and hence

$$
\mathfrak{D}_{k}=\mathfrak{D}_{k-1} \cup \bigcup_{p} \mathfrak{D}[p]
$$

has the desired properties.

We take $\mathbf{S}(\mathfrak{D})$ to be the (non-disjoint) union of the $\mathfrak{D}_{k}$ 's. The diagram $\mathbf{S}(\mathfrak{D})$ will usually have infinitely many rays even if $\mathfrak{D}$ has finitely many.

The scattering diagram $\mathbf{S}(\mathfrak{D})$ is not unique. There are always trivial changes which can be made to scattering diagrams.

Definition 1.5. Two scattering diagrams $\mathfrak{D}, \mathfrak{D}^{\prime}$ over a ring $R$ are equivalent if

$$
\theta_{\gamma, \mathfrak{D}}=\theta_{\gamma, \mathfrak{D}^{\prime}}
$$

for every curve $\gamma$ for which both sides are defined.

Every scattering diagram $\mathfrak{D}$ is equivalent to a unique minimal scattering diagram which does not have two rays or lines with the same support and contains no trivial rays or lines - rays or lines $\left(\mathfrak{d}, f_{\mathfrak{d}}\right)$ with $f_{\mathfrak{d}}=1$. In fact, if $\left(\mathfrak{d}_{1}, f_{1}\right),\left(\mathfrak{d}_{2}, f_{2}\right) \in \mathfrak{D}$ with $\mathfrak{d}_{1}=\mathfrak{d}_{2}$, then we can replace these two rays or lines with the single ray or line $\left(\mathfrak{d}_{1}, f_{1} f_{2}\right)$ without affecting any automorphism $\theta_{\gamma, \mathfrak{D}}$. In addition we can remove any trivial ray or line. The diagram $S(\mathfrak{D})$ is easily seen to be unique up to equivalence. We will often assume $\mathbf{S}(\mathfrak{D})$ is minimal.

The operation $\mathrm{S}$ is functorial in the following sense. If $\varphi: R \rightarrow R^{\prime}$ is a ring homomorphism, and

$$
\varphi(\mathfrak{D})=\left\{\left(\mathfrak{d}, \varphi\left(f_{\mathfrak{d}}\right)\right) \mid\left(\mathfrak{d}, f_{\mathfrak{d}}\right) \in \mathfrak{D}\right\},
$$

then $\varphi(\mathbf{S}(\mathfrak{D}))$ is equivalent to $S(\varphi(\mathfrak{D}))$. 


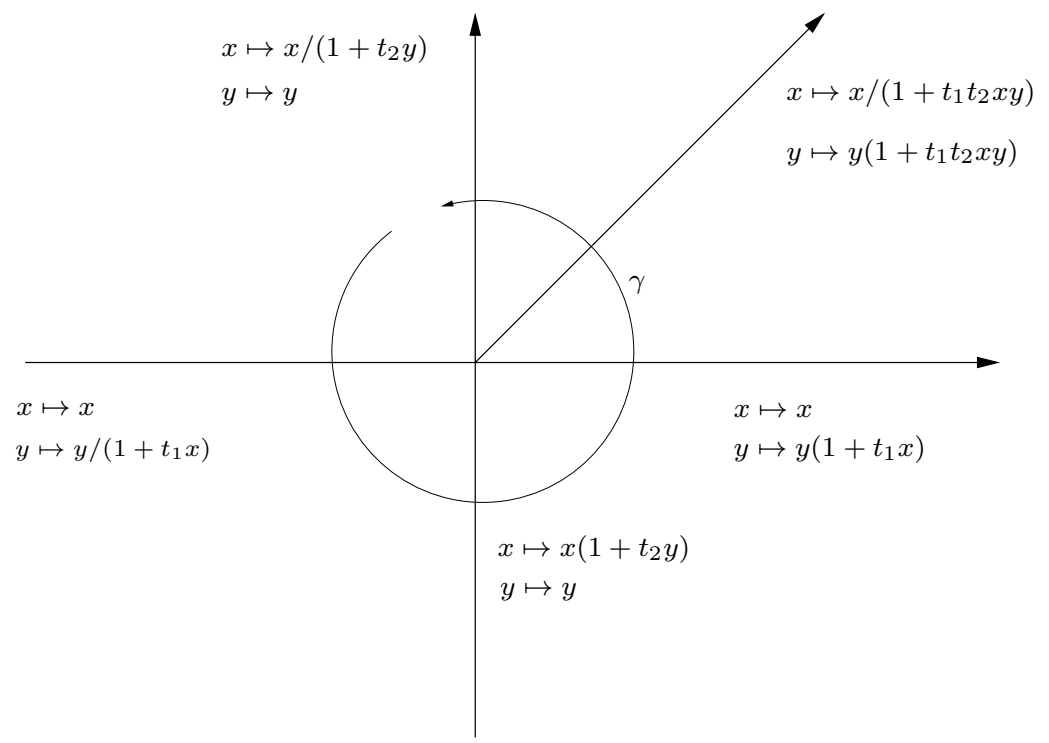

FiguRE 1.2. $\mathrm{S}(\mathfrak{D})$ for $\ell_{1}=1, \ell_{2}=1$. Here the automorphisms are given explicitly, and the identity $\theta_{\gamma, \mathrm{S}(\mathfrak{D})}$ is just the composition of the given automorphisms.

Example 1.6. Let $R=\mathbb{C}\left[\left[t_{1}, t_{2}\right]\right]$ and consider for $\ell_{1}, \ell_{2}>0$ the diagram

$$
\mathfrak{D}=\left\{\left(\mathbb{R}(1,0),\left(1+t_{1} x\right)^{\ell_{1}}\right),\left(\mathbb{R}(0,1),\left(1+t_{2} y\right)^{\ell_{2}}\right)\right\} .
$$

Here, $x=z^{e_{1}}$ and $y=z^{e_{2}}$ as before. Theorem 1.4 produces a scattering diagram $\mathrm{S}(\mathfrak{D})$. We will later see that all elements of $S(\mathfrak{D}) \backslash \mathfrak{D}$ lie in the first quadrant. However, $\mathrm{S}(\mathfrak{D}) \backslash \mathfrak{D}$ can be very complicated 6

If $\ell_{1}=\ell_{2}=1$, then $S(\mathfrak{D})$ requires only a single additional ray,

$$
\mathbf{S}(\mathfrak{D}) \backslash \mathfrak{D}=\left\{\left(\mathbb{R}(1,1), 1+t_{1} t_{2} x y\right)\right\},
$$

as can be easily checked by hand. See Figure 1.2 .

If $\ell_{1}=\ell_{2}=2$, then the following is found:

$$
\begin{aligned}
& \mathrm{S}(\mathfrak{D}) \backslash \mathfrak{D}=\quad\left\{\left(\mathbb{R}(n+1, n),\left(1+\left(t_{1} x\right)^{n+1}\left(t_{2} y\right)^{n}\right)^{2}\right) \mid n \in \mathbb{Z}, n \geq 1\right\} \\
& \cup \quad\left\{\left(\mathbb{R}(n, n+1),\left(1+\left(t_{1} x\right)^{n}\left(t_{2} y\right)^{n+1}\right)^{2}\right) \mid n \in \mathbb{Z}, n \geq 1\right\} \\
& \cup \quad\left\{\left(\mathbb{R}(1,1),\left(1-t_{1} t_{2} x y\right)^{-4}\right)\right\} .
\end{aligned}
$$

If $\ell_{1}=\ell_{2}=3$, then computer experiments (the algorithm given in the proof of Theorem 1.4 is easily implemented) suggest the following behavior for $S(\mathfrak{D})$. First, as noticed by Kontsevich, $\mathbf{S}(\mathfrak{D})$ has a certain periodicity. Namely,

$$
\left(\mathbb{R}_{\geq 0}\left(m_{1}, m_{2}\right), f\left(z^{\left(m_{1}, m_{2}\right)}\right)\right) \in \mathbf{S}(\mathfrak{D})
$$

\footnotetext{
${ }^{6}$ The behavior of these scattering diagrams has also been studied by Kontsevich.
} 
if and only if

$$
\left(\mathbb{R}_{\geq 0}\left(3 m_{1}-m_{2}, m_{1}\right), f\left(z^{\left(3 m_{1}-m_{2}, m_{1}\right)}\right)\right) \in \mathbf{S}(\mathfrak{D}),
$$

provided that $m_{1}, m_{2}$ and $3 m_{1}-m_{2}$ are all positive. In addition, there are rays with support $\mathbb{R}_{\geq 0}(3,1)$ and $\mathbb{R}_{\geq 0}(1,3)$, hence by the periodicity, there are also rays with support

$$
\mathbb{R}_{\geq 0}(8,3), \mathbb{R}_{\geq 0}(21,8), \ldots \quad \text { and } \quad \mathbb{R}_{\geq 0}(3,8), \mathbb{R}_{\geq 0}(8,21), \ldots
$$

which converge to the rays of slope $(3 \pm \sqrt{5}) / 2$, corresponding to the two distinct eigenspaces of the linear transformation $\left(\begin{array}{cc}3 & -1 \\ 1 & 0\end{array}\right)$. On the other hand, inside the cone generated by the rays of slope $(3 \pm \sqrt{5}) / 2$, it appears that every rational slope occurs. The function attached to the line of slope 1 appears to be

$$
\left(\sum_{n=0}^{\infty} \frac{1}{3 n+1}\left(\begin{array}{c}
4 n \\
n
\end{array}\right)\left(t_{1} x\right)^{n}\left(t_{2} y\right)^{n}\right)^{9}
$$

1.4. Asymptotic diagrams and factorization. A scattering diagram $\mathfrak{D}$ viewed from a great distance yields an asymptotic diagram.

Definition 1.7. The asymptotic scattering diagram $\mathfrak{D}_{\text {as }}$ is obtained from $\mathfrak{D}$ by replacing each ray $\left(m_{0}^{\prime}+\mathbb{R}_{\geq 0} m_{0}, f\right)$ with the ray $\left(\mathbb{R}_{\geq 0} m_{0}, f\right)$ emanating from the origin, and replacing each line $\left(m_{0}^{\prime}+\mathbb{R} m_{0}, f\right)$ with the line $\left(\mathbb{R} m_{0}, f\right)$ passing through the origin.

If $\gamma$ is a sufficiently large simple loop around the origin containing all points of $\operatorname{Sing}(\mathfrak{D})$, then

$$
\theta_{\gamma, \mathfrak{D}}=\theta_{\gamma, \mathfrak{D}_{a s}}
$$

We can often understand scattering diagrams using a deformation technique as follows. Suppose $\mathfrak{D}$ consists of a number of lines $\left(\mathfrak{d}_{i}, f_{i}\right)$ through the origin, and we wish to understand $\mathbf{S}(\mathfrak{D})$. Assume that each $f_{i}$ can be factored as $f_{i}=\prod f_{i j}$. We replace each line $\left(\mathfrak{d}_{i}, f_{i}\right)$ with the collection of lines $\left\{\left(m_{i j}^{\prime}+\mathfrak{d}_{i}, f_{i j}\right)\right\}$, where $m_{i j}^{\prime} \in M_{\mathbb{R}}$ is chosen generally. Thus each line is split up into a number of parallel lines. Calling this new scattering diagram $\widetilde{\mathfrak{D}}$, we can now apply Theorem 1.4 to obtain a scattering diagram $\mathrm{S}(\widetilde{\mathfrak{D}})$. Then $\mathrm{S}(\widetilde{\mathfrak{D}})_{\text {as }}$ satisfies the conclusion of Theorem 1.4 when applied to $\mathfrak{D}$, since for a large simple loop around the origin, $\theta_{\gamma, \mathrm{S}(\widetilde{\mathfrak{D}})_{a s}}=\mathrm{Id}$.

For example, in Example 1.6, we can split the two lines in $\mathfrak{D}$ into $\ell_{1}$ and $\ell_{2}$ parallel lines respectively.

Definition 1.8. If $m \in M \backslash\{0\}$, the index of $m$ is a positive integer $w$ such that $m=w m^{\prime}$ for $m^{\prime} \in M$ primitive. 
The following simple calculation is closely related to the notion of multiplicity in tropical geometry.

Lemma 1.9. Let $R=\mathbb{C}\left[t_{1}, t_{2}\right] /\left(t_{1}^{2}, t_{2}^{2}\right)$, and let

$$
\mathfrak{D}=\left\{\left(\mathbb{R} m_{1}, 1+c_{1} t_{1} z^{w_{1} m_{1}}\right),\left(\mathbb{R} m_{2}, 1+c_{2} t_{2} z^{w_{2} m_{2}}\right)\right\}
$$

where $m_{1}, m_{2} \in M$ are primitive with $m_{1} \wedge m_{2} \neq 0, w_{1}, w_{2}$ are positive integers and $c_{1}, c_{2} \in \mathbb{C}$. The scattering diagram $\mathbf{S}(\mathfrak{D})$ of Theorem 1.4 is obtained by adding the single ray

$$
\left(\mathbb{R}_{\geq 0}\left(w_{1} m_{1}+w_{2} m_{2}\right), 1+c_{1} c_{2} t_{1} t_{2} w_{\text {out }}\left|m_{1} \wedge m_{2}\right| z^{w_{1} m_{1}+w_{2} m_{2}}\right)
$$

where $\left|m_{1} \wedge m_{2}\right|$ denotes the absolute value of $m_{1} \wedge m_{2} \in \wedge^{2} M \cong \mathbb{Z}$, and $w_{\text {out }}$ is the index of $w_{1} m_{1}+w_{2} m_{2}$.

If $w_{1} m_{1}+w_{2} m_{2}=0$ in (1.2), no ray is added. The associated function then is just 1 since then $m_{1} \wedge m_{2}=0$.

Proof. Labelling the two rays $\mathfrak{d}_{1}$ and $\mathfrak{d}_{2}$ respectively, choose a loop $\gamma$ as depicted in Figure 1.1, so that

$$
\theta_{\gamma, \mathfrak{D}}=\theta_{2}^{-1} \theta_{1}^{-1} \theta_{2} \theta_{1}
$$

where

$$
\theta_{i}=\exp \left(c_{i} t_{i} z^{w_{i} m_{i}} \partial_{n_{i}}\right)
$$

with $n_{i} \in N$ primitive, orthogonal to $m_{i}, n_{1}$ negative on $m_{2}$ and $n_{2}$ positive on $m_{1}$. The commutator is easily seen from (1.1) or direct computation to be

$$
\exp \left(c_{1} c_{2} t_{1} t_{2} z^{w_{1} m_{1}+w_{2} m_{2}} \partial_{w_{1} n_{1}\left\langle m_{1}, n_{2}\right\rangle-w_{2} n_{2}\left\langle m_{2}, n_{1}\right\rangle}\right) .
$$

Thus $\mathbf{S}(\mathfrak{D})$ needs to include exactly one additional ray, $\left(\mathbb{R}_{\geq 0}\left(w_{1} m_{1}+w_{2} m_{2}\right)\right.$, $\left.f\right)$, with $f$ chosen so the corresponding automorphism determined by the crossing of $\gamma$ is the inverse of (1.3).

First assume the basis $m_{1}, m_{2}$ of $M_{\mathbb{R}}$ is positively oriented, as in Figure 1.1. If we write $m_{i}=\left(m_{i 1}, m_{i 2}\right)$ in some fixed basis of $M$, we can take $n_{i}=\left(m_{i 2},-m_{i 1}\right)$ in the dual basis. Then,

$$
\left\langle m_{1}, n_{2}\right\rangle=-\left\langle m_{2}, n_{1}\right\rangle=m_{11} m_{22}-m_{12} m_{21}=\left|m_{1} \wedge m_{2}\right|
$$

because of the positive orientation. So

$$
\begin{aligned}
w_{1} n_{1}\left\langle m_{1}, n_{2}\right\rangle-w_{2} n_{2}\left\langle m_{2}, n_{1}\right\rangle & =\left|m_{1} \wedge m_{2}\right|\left(w_{1} m_{12}+w_{2} m_{22},-w_{1} m_{11}-w_{2} m_{21}\right) \\
& =\left|m_{1} \wedge m_{2}\right| w_{\text {out }} n_{\text {out }}
\end{aligned}
$$

where $n_{\text {out }}$ is a primitive element of $N$ pointing in the same direction as

$$
w_{1} n_{1}\left\langle m_{1}, n_{2}\right\rangle-w_{2} n_{2}\left\langle m_{2}, n_{1}\right\rangle .
$$


The automorphism we wish to attach to the new ray is

$$
\exp \left(c_{1} c_{2} t_{1} t_{2} w_{\text {out }}\left|m_{1} \wedge m_{2}\right| z^{w_{1} m_{1}+w_{2} m_{2}} \partial_{-n_{\text {out }}}\right)
$$

Noting that $-n_{\text {out }}$ is positive on $m_{2}$, we see that we can take

$$
f=1+c_{1} c_{2} t_{1} t_{2} w_{\text {out }}\left|m_{1} \wedge m_{2}\right| z^{w_{1} m_{1}+w_{2} m_{2}} .
$$

as claimed.

If $m_{1}, m_{2}$ is negatively oriented, the argument is similar. Or, we can reduce to the positively oriented case by switching labels.

In fact, using factorization and deformation, Lemma 1.9 is enough to understand a scattering diagram to any order. We will demonstrate the method for the scattering diagrams we require for the remainder of the paper, so the notation introduced here will be used throughout.

Definition 1.10. A standard scattering diagram $\mathfrak{D}=\left\{\left(\mathfrak{d}_{i}, f_{i}\right) \mid 1 \leq i \leq n\right\}$ over the $\operatorname{ring} R=\mathbb{C}\left[\left[t_{1}, \ldots, t_{n}\right]\right]$ consists of a number of lines through the origin. We assume

- $\mathfrak{d}_{i}=\mathbb{R} m_{i}$ with $m_{i}$ primitive 7

- $t_{i}$ is the only power series variable occurring in $f_{i}$, so

$$
f_{i} \in \mathbb{C}\left[z^{m_{i}}\right] \widehat{\otimes}_{\mathbb{C}} \mathbb{C}\left[\left[t_{i}\right]\right]
$$

We give here a method of computing $\mathbf{S}(\mathfrak{D})$ to $k$ th order for a standard diagram $\mathfrak{D}$. We work over the ring

$$
R_{k}=\frac{\mathbb{C}\left[\left[t_{1}, \ldots, t_{n}\right]\right]}{\left(t_{1}^{k+1}, \ldots, t_{n}^{k+1}\right)} .
$$

Let $\alpha_{k}: R \rightarrow R_{k}$ be the projection, and define

$$
\begin{aligned}
\mathrm{S}_{k}(\mathfrak{D}) & =\mathbf{S}\left(\alpha_{k}(\mathfrak{D})\right) \\
& =\alpha_{k}(\mathbf{S}(\mathfrak{D})) .
\end{aligned}
$$

We view $S_{k}(\mathfrak{D})$ as $\mathbf{S}(\mathfrak{D})$ to $k$ th order.

A crucial role is played by the ring

$$
\widetilde{R}_{k}=\frac{\mathbb{C}\left[\left\{u_{i j} \mid 1 \leq i \leq n, 1 \leq j \leq k\right\}\right]}{\left\langle u_{i j}^{2} \mid 1 \leq i \leq n, 1 \leq j \leq k\right\rangle} .
$$

via the basic inclusion $R_{k} \hookrightarrow \widetilde{R}_{k}$ given by $t_{i} \mapsto \sum_{j=1}^{k} u_{i j}$. While the $f_{i}$ 's may not factor over $R_{k}$, there is a natural factorization of $f_{i}$ over $\widetilde{R}_{k}$ obtained as follows. We expand

\footnotetext{
${ }^{7}$ We do not assume the $m_{i}$ are distinct. Repetition is allowed.
} 
$\log f_{i}$ in a Taylor series in $t_{i}$

$$
\log f_{i}=\sum_{j=1}^{k} \sum_{w \geq 1} w a_{i j w} z^{w m_{i}} t_{i}^{j} \in R_{k}
$$

with $a_{i j w} \in \mathbb{C}$. For any given $i$ and $j, a_{i j w}=0$ for all but a finite number of $w$. We then make the substitution $t_{i}=\sum_{j=1}^{k} u_{i j}$ and expand, getting a sum of monomials each having a coefficient in $\widetilde{R}_{k}$ whose square is zero. We find

$$
\log f_{i}=\sum_{j=1}^{k} \sum_{\# J=j} \sum_{w \geq 1} j ! w a_{i j w} z^{w m_{i}} \prod_{l \in J} u_{i l}
$$

where $J$ runs over subsets of $\{1, \ldots, k\}$. Taking the exponential gives us a factorization of $f_{i}$ as

$$
f_{i}=\prod_{j=1}^{k} \prod_{\# J=j} \prod_{w \geq 1}\left(1+j ! w a_{i j w} z^{w m_{i}} \prod_{l \in J} u_{i l}\right)
$$

We can now apply the deformation technique to the factorization to pull apart the lines $\mathfrak{d}_{i}$. We obtain the scattering diagram

$\widetilde{\mathfrak{D}}_{k}=\left\{\left(\mathfrak{d}_{i J w}, 1+(\# J) ! w a_{i(\# J) w} z^{w m_{i}} \prod_{l \in J} u_{i l}\right) \mid 1 \leq i \leq n, w \geq 1, J \subseteq\{1, \ldots, k\}, \# J \geq 1\right\}$, with $\mathfrak{d}_{i J w}$ a line parallel to $\mathfrak{d}_{i}$ and chosen generally.

We now describe rather concretely a process for producing $\mathbf{S}\left(\widetilde{\mathfrak{D}}_{k}\right)$. We usually only considered $\mathbf{S}\left(\widetilde{\mathfrak{D}}_{k}\right)$ as an equivalence class of scattering diagrams. However, in Section 2 , we will use the representative of the equivalence class produced by the procedure given here.

We produce a sequence of scattering diagrams

$$
\widetilde{\mathfrak{D}}_{k}=\widetilde{\mathfrak{D}}_{k}^{0}, \widetilde{\mathfrak{D}}_{k}^{1}, \widetilde{\mathfrak{D}}_{k}^{2}, \ldots
$$

which eventually stabilizes, and we take $\mathbf{S}\left(\widetilde{\mathfrak{D}}_{k}\right)=\widetilde{\mathfrak{D}}_{k}^{i}$ for $i$ sufficiently large. We will assume inductively:

(1) Each ray or line in $\widetilde{\mathfrak{D}}_{k}^{i}$ is of the form

$$
\left(\mathfrak{d}, 1+c_{\mathfrak{d}} u_{I(\mathfrak{d})} z^{m_{\mathfrak{d}}}\right),
$$

with $c_{\mathfrak{d}} \in \mathbb{C}$ and $u_{I(\mathfrak{d})}=\prod_{(i, j) \in I(\mathfrak{d})} u_{i j}$, for some index set

$$
I(\mathfrak{d}) \subseteq\{1, \ldots, n\} \times\{1, \ldots, k\} .
$$

(2) For each $p \in \operatorname{Sing}\left(\widetilde{\mathfrak{D}}_{k}^{i}\right)$, there is no set of rays $\mathfrak{D}[p] \subseteq \widetilde{\mathfrak{D}}_{k}^{i}$ of cardinality $\geq 3$ such that $p \in \mathfrak{d}$ for each $\mathfrak{d} \in \mathfrak{D}[p]$ and $I\left(\mathfrak{d}_{1}\right) \cap I\left(\mathfrak{d}_{2}\right)=\emptyset$ for any two distinct $\mathfrak{d}_{1}, \mathfrak{d}_{2} \in \mathfrak{D}[p]$. Note that two rays $\mathfrak{d}_{1}, \mathfrak{d}_{2}$ only produce a non-trivial new ray via Lemma 1.9 if $I\left(\mathfrak{d}_{1}\right) \cap I\left(\mathfrak{d}_{2}\right)=\emptyset$. 
Both of these conditions already hold for $\widetilde{\mathfrak{D}}_{k}^{0}$. In particular, for general choice of $\widetilde{\mathfrak{D}}_{k}^{0}$, each singular point of $\widetilde{\mathfrak{D}}_{k}^{0}$ is contained in only two lines.

To pass from $\widetilde{\mathfrak{D}}_{k}^{i-1}$ to $\widetilde{\mathfrak{D}}_{k}^{i}$, we simply look at every pair of elements $\mathfrak{d}_{1}, \mathfrak{d}_{2} \in \widetilde{\mathfrak{D}}_{k}^{i-1}$ satisfying

(i) $\left\{\mathfrak{d}_{1}, \mathfrak{d}_{2}\right\} \nsubseteq \widetilde{\mathfrak{D}}_{k}^{i-2}$,

(ii) $\mathfrak{d}_{1} \cap \mathfrak{d}_{2}$ consists of one point $m_{0}$, which is neither the boundary of $\mathfrak{d}_{1}$ nor $\mathfrak{d}_{2}$,

(iii) $I\left(\mathfrak{d}_{1}\right) \cap I\left(\mathfrak{d}_{2}\right)=\emptyset$.

Writing

$$
f_{\mathfrak{o}_{i}}=1+c_{i} u_{I\left(\mathfrak{o}_{i}\right)} z^{w_{i} m_{i}}
$$

with $m_{i}$ primitive, we follow Lemma 1.9 and set

$$
\begin{aligned}
& \mathfrak{d}\left(\mathfrak{d}_{1}, \mathfrak{d}_{2}\right)= \\
& \quad\left(m_{0}+\mathbb{R}_{\geq 0}\left(w_{1} m_{1}+w_{2} m_{2}\right), 1+c_{1} c_{2} u_{I\left(\mathfrak{d}_{1}\right) \cup I\left(\mathfrak{d}_{2}\right)} w_{\text {out }}\left|m_{1} \wedge m_{2}\right| z^{w_{1} m_{1}+w_{2} m_{2}}\right) .
\end{aligned}
$$

We then take

$$
\widetilde{\mathfrak{D}}_{k}^{i}=\widetilde{\mathfrak{D}}_{k}^{i-1} \cup\left\{\mathfrak{d}\left(\mathfrak{d}_{1}, \mathfrak{d}_{2}\right) \mid \mathfrak{d}_{1}, \mathfrak{d}_{2} \text { satisfies (i)-(iii) above }\right\} .
$$

Of course $\widetilde{\mathfrak{D}}_{k}^{i}$ satisfies the inductive hypothesis (1), but we need to check condition (2). To do so, we introduce some notation we shall also need later.

For any ray of the form $\mathfrak{d}=\mathfrak{d}\left(\mathfrak{d}_{1}, \mathfrak{d}_{2}\right)$, define Parents $(\mathfrak{d})=\left\{\mathfrak{d}_{1}, \mathfrak{d}_{2}\right\}$. If $\mathfrak{d}$ is a line, then we set Parents $(\mathfrak{d})=\emptyset$. Define recursively

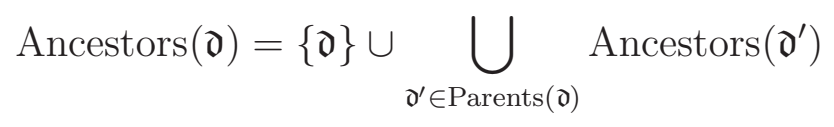

and

$$
\operatorname{Leaves}(\mathfrak{d})=\left\{\mathfrak{d}^{\prime} \in \operatorname{Ancestors}(\mathfrak{d}) \mid \mathfrak{d}^{\prime} \text { is a line }\right\} .
$$

If any element $\mathfrak{d}^{\prime} \in$ Leaves $(\mathfrak{d})$ is moved slightly by replacing $\mathfrak{d}^{\prime}=m+\mathbb{R} m_{i}$ by $m^{\prime}+\mathbb{R} m_{i}$ with $m-m^{\prime}$ small and $m-m^{\prime} \notin \mathbb{R} m_{i}$, then $\mathfrak{d}$ is replaced by some $m^{\prime \prime}+\mathfrak{d}$ for $m^{\prime \prime}$ small and $m^{\prime \prime} \notin \mathbb{R} m_{\mathfrak{d}}$. Now if there is a point $p \in \operatorname{Sing}\left(\widetilde{\mathfrak{D}}_{k}^{i}\right)$ with $\mathfrak{D}[p] \subseteq \widetilde{\mathfrak{D}}_{k}^{i}$ violating condition (2), we note Leaves $\left(\mathfrak{d}_{1}\right) \cap \operatorname{Leaves}\left(\mathfrak{d}_{2}\right)=\emptyset$ for all $\mathfrak{d}_{1}, \mathfrak{d}_{2} \in \mathfrak{D}[p]$, since $I\left(\mathfrak{d}_{1}\right) \cap I\left(\mathfrak{d}_{2}\right)=\emptyset$. Thus by deforming the lines in $\widetilde{\mathfrak{D}}_{k}^{0}$, the rays in $\mathfrak{D}[p]$ can be deformed in an independent manner. Thus if $p$ violates $(2)$, then $\widetilde{\mathfrak{D}}_{k}^{0}$ has not been chosen generally. Thus for general choice of $\widetilde{\mathfrak{D}}_{k}^{0}$, the inductive hypothesis (2) holds.

The process is easily seen to terminate as at each step the sets $I(\mathfrak{d})$ for new rays $\mathfrak{d}$ increase in cardinality, and the cardinality of these sets is bounded by $n k$. Furthermore, since automorphisms associated to rays $\mathfrak{d}_{1}$, $\mathfrak{d}_{2}$ with $I\left(\mathfrak{d}_{1}\right) \cap I\left(\mathfrak{d}_{2}\right) \neq \emptyset$ commute, we obtain from Lemma 1.9 that $\theta_{\gamma_{p}, \widetilde{\mathfrak{D}}_{k}^{i}}=\operatorname{Id}$ for $i>n k$ and $\gamma_{p}$ a small loop around any singular point of $\widetilde{\mathfrak{D}}_{k}^{i}$. 
We have proven $\mathbf{S}\left(\widetilde{\mathfrak{D}}_{k}\right)=\widetilde{\mathfrak{D}}_{k}^{\infty}$ where stabilization occurs in the superscript for $i>n k$. Then

$$
\mathrm{S}_{k}(\mathfrak{D})=\left(\widetilde{\mathfrak{D}}_{k}^{\infty}\right)_{\text {as }}
$$

As we will see in the next section, we will use the above procedure to reduce the computation of $S_{k}(\mathfrak{D})$ to a tropical calculation.

Example 1.11. We apply the above procedure to Example 1.6 with $\ell_{1}=\ell_{2}=1$ and

$$
f_{1}=1+t_{1} x, \quad f_{2}=1+t_{2} y .
$$

For $k=2$, we write

$$
\log f_{1}=t_{1} x-\frac{t_{1}^{2} x^{2}}{2}+\cdots=u_{11} x+u_{12} x-u_{11} u_{12} x^{2}
$$

and factor $f_{1}$ as

$$
f_{1}=\left(1+u_{11} x\right)\left(1+u_{12} x\right)\left(1-u_{11} u_{12} x^{2}\right)
$$

Similarly,

$$
f_{2}=\left(1+u_{21} y\right)\left(1+u_{22} y\right)\left(1-u_{21} u_{22} y^{2}\right) .
$$

Applying the above procedure gives the complicated scattering diagram depicted in Figure 1.3 .

In the scattering diagram, there are four rays of slope 2, eight of slope 1 , and four of slope $1 / 2$. That the asymptotic form of the diagram agrees with Figure 1.2 appears miraculous. To see the agreement, we calculate the functions attached to the various rays in Figure 1.3 using Lemma 1.9. For example, the rays of slope 2 have attached functions, taken in a clockwise order,

$$
1+u_{11} u_{21} u_{22} x y^{2}, 1+u_{12} u_{21} u_{22} x y^{2}, 1-u_{11} u_{21} u_{22} x y^{2}, 1-u_{12} u_{21} u_{22} x y^{2} .
$$

The product of these four functions is 1, so the ray in the asymptotic diagram associated to this diagram has the attached function 1 , and hence can be omitted. The same happens to the rays of slope $1 / 2$. For the rays of slope 1 , again in clockwise order, again using Lemma 1.9, we have the attached functions

$$
\begin{gathered}
1+u_{11} u_{21} x y, 1+u_{11} u_{22} x y, 1+4 u_{11} u_{12} u_{21} u_{22} x^{2} y^{2}, 1+u_{12} u_{21} x y, 1+u_{12} u_{22} x y \\
1-4 u_{11} u_{12} u_{21} u_{22} x^{2} y^{2}, 1-4 u_{11} u_{12} u_{21} u_{22} x^{2} y^{2}, 1+2 u_{11} u_{12} u_{21} u_{22} x^{2} y^{2}
\end{gathered}
$$

Taking the product of these eight functions gives $1+\left(u_{11}+u_{12}\right)\left(u_{21}+u_{22}\right) x y$. All the terms involving $x^{2} y^{2}$ cancel. 


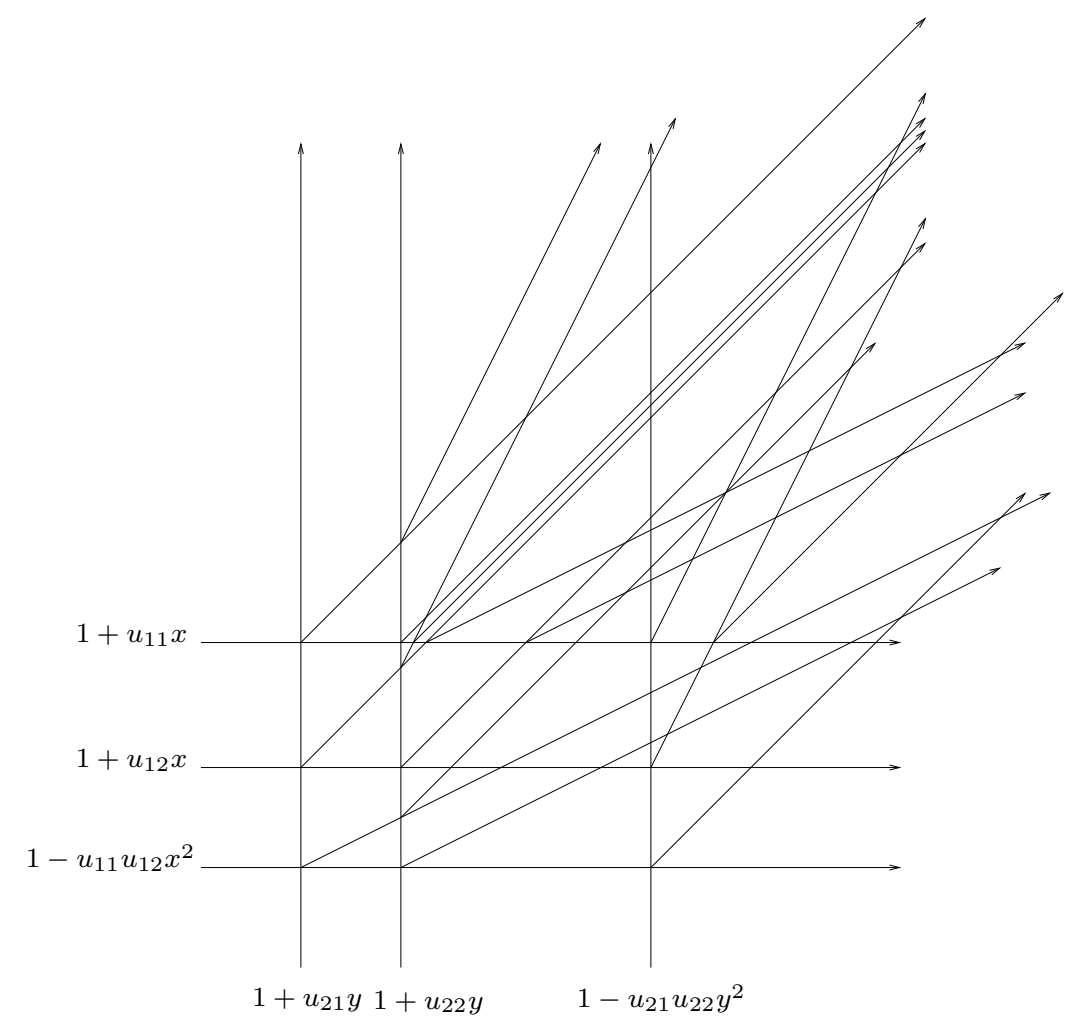

Figure 1.3.

\section{Tropical CURVES}

2.1. Definitions. We relate here the calculations involving scattering diagrams in Section 1.4 to tropical curve counts. We first recall the definition of a parameterized tropical curve in $M_{\mathbb{R}}$ from [19]. Let $\bar{\Gamma}$ be a weighted, connected finite graph without divalent vertices. Denote the set of vertices and edges by $\bar{\Gamma}^{[0]}$ and $\bar{\Gamma}^{[1]}$ respectively, and let

$$
w_{\bar{\Gamma}}: \bar{\Gamma}^{[1]} \rightarrow \mathbb{N} \backslash\{0\}
$$

be the weight function. An edge $E \in \bar{\Gamma}^{[1]}$ has adjacent vertices $\partial E=\left\{V_{1}, V_{2}\right\}$. Let $\bar{\Gamma}_{\infty}^{[0]} \subseteq \bar{\Gamma}^{[0]}$ be the set of 1 -valent vertices. We set

$$
\Gamma=\bar{\Gamma} \backslash \bar{\Gamma}_{\infty}^{[0]}
$$

Denote the set of vertices and edges of $\Gamma$ as $\Gamma^{[0]}, \Gamma^{[1]}$, and let

$$
w_{\Gamma}: \Gamma^{[1]} \rightarrow \mathbb{N} \backslash\{0\}
$$

be the weight function. Some edges of $\Gamma$ are now non-compact - these are called unbounded edges. Let $\Gamma_{\infty}^{[1]} \subseteq \Gamma^{[1]}$ be the set of unbounded edges. 
Definition 2.1. A parameterized tropical curve in $M_{\mathbb{R}}$ is a proper map $h: \Gamma \rightarrow M_{\mathbb{R}}$ satisfying the following conditions.

(1) For every edge $E \subseteq \Gamma$, the restriction $\left.h\right|_{E}$ is an embedding with image $h(E)$ contained in an affine line with rational slope.

(2) For every vertex $V \in \Gamma$, the following balancing condition holds. Let

$$
E_{1}, \ldots, E_{m} \in \Gamma^{[1]}
$$

be the edges adjacent to $V$, and let $m_{i} \in M$ be the primitive integral vector emanating from $h(V)$ in the direction of $h\left(E_{i}\right)$. Then

$$
\sum_{j=1}^{m} w_{\Gamma}\left(E_{j}\right) m_{j}=0 .
$$

An isomorphism of parameterized tropical curves $h: \Gamma \rightarrow M_{\mathbb{R}}$ and $h^{\prime}: \Gamma^{\prime} \rightarrow M_{\mathbb{R}}$ is a homeomorphism $\Phi: \Gamma \rightarrow \Gamma^{\prime}$ respecting the weights of the edges and such that $h=h^{\prime} \circ \Phi$. A tropical curve is an isomorphism class of parameterized tropical curves. The genus of a tropical curve $h: \Gamma \rightarrow M_{\mathbb{R}}$ is the first Betti number of $\Gamma$. A rational tropical curve is a tropical curve of genus zero.

Definition 2.2. Let $h: \Gamma \rightarrow M_{\mathbb{R}}$ be a tropical curve such that $\bar{\Gamma}$ only has vertices of valency one and three. The multiplicity of a vertex $V \in \Gamma^{[0]}$ in $h$ is

$$
\operatorname{Mult}_{V}(h)=w_{1} w_{2}\left|m_{1} \wedge m_{2}\right|=w_{1} w_{3}\left|m_{1} \wedge m_{3}\right|=w_{2} w_{3}\left|m_{2} \wedge m_{3}\right|,
$$

where $E_{1}, E_{2}, E_{3} \in \Gamma^{[1]}$ are the edges containing $V, w_{i}=w_{\Gamma}\left(E_{i}\right)$, and $m_{i} \in M$ is a primitive integral vector emanating from $h(V)$ in the direction of $h\left(E_{i}\right)$. The equality of the three expressions follows from the balancing condition.

Definition 2.3. The multiplicity of the tropical curve $h$ is

$$
\operatorname{Mult}(h)=\prod_{V \in \Gamma^{[0]}} \operatorname{Mult}_{V}(h) .
$$

2.2. Scattering diagrams. Let $\mathfrak{D}=\left\{\left(\mathbb{R} m_{i}, f_{i}\right)\right\}$ be a standard scattering diagram of Definition 1.10. We follow the notation of Section 1.4 with scattering diagrams $\widetilde{\mathfrak{D}}_{k}$ and

$$
\mathrm{S}\left(\widetilde{\mathfrak{D}}_{k}\right)=\widetilde{\mathfrak{D}}_{k}^{\infty}
$$

constructed from $\mathfrak{D}$.

Theorem 2.4. For general choice of $\widetilde{\mathfrak{D}}_{k}$, there is a bijective correspondence between elements $\left(\mathfrak{d}, f_{\mathfrak{d}}\right) \in \mathrm{S}\left(\widetilde{\mathfrak{D}}_{k}\right)$ and rational tropical curves $h: \Gamma \rightarrow M_{\mathbb{R}}$ with the following properties:

(i) There is an edge $E_{\text {out }} \in \Gamma_{\infty}^{[1]}$ with $h\left(E_{\text {out }}\right)=\mathfrak{d}$. 
(ii) If $E \in \Gamma_{\infty}^{[1]} \backslash\left\{E_{\text {out }}\right\}$ or if $E_{\text {out }}$ is the only edge of $\Gamma$ and $E=E_{\text {out }}$, then $h(E)$ is contained in some $\mathfrak{d}_{i J w}$ where

$$
1 \leq i \leq n, \quad J \subseteq\{1, \ldots, k\}, \quad w \geq 1
$$

Furthermore, if $E \neq E_{\text {out }}$, the unbounded direction of $h(E)$ is given by $-m_{i}$.

(iii) If $E, E^{\prime} \in \Gamma_{\infty}^{[1]} \backslash\left\{E_{\text {out }}\right\}$ and $h(E) \subseteq \mathfrak{d}_{i J w}$ and $h\left(E^{\prime}\right) \subseteq \mathfrak{d}_{i J^{\prime} w^{\prime}}$, then $J \cap J^{\prime}=\emptyset$.

(iv) If $E \in \Gamma_{\infty}^{[1]} \backslash\left\{E_{\text {out }}\right\}$ or if $E_{\text {out }}$ is the only edge of $\Gamma$ and $E=E_{\text {out }}$, and $h(E) \subseteq$ $\mathfrak{d}_{i J w}$, we have $w_{\Gamma}(E)=w$.

Furthermore, if $\mathfrak{d}$ is a ray, the corresponding curve $h$ is trivalent and

$$
f_{\mathfrak{o}}=1+w_{\text {out }} \operatorname{Mult}(h) \prod_{i, J, w}\left((\# J) ! a_{i(\# J) w} \prod_{j \in J} u_{i j}\right) z^{m_{\text {out }}},
$$

where the $i, J, w$ run over all indices for which $\mathfrak{d}_{i J w} \in \operatorname{Leaves}(\mathfrak{d})$,

$$
m_{\mathrm{out}}=\sum_{i, J, w} w m_{i}
$$

and $m_{\mathrm{out}}=w_{\mathrm{out}} m_{\mathrm{out}}^{\prime}$ for $m_{\mathrm{out}}^{\prime} \in M$ primitive and $w_{\mathrm{out}}$ the index of $m_{\mathrm{out}}$.

Proof. The bijective correspondence is constructed as follows. First, the elements of $\widetilde{\mathfrak{D}}_{k}$ are all lines and are clearly in one-to-one correspondence with tropical curves $h$ : $\Gamma \rightarrow M_{\mathbb{R}}$ in which $\Gamma$ consists of just one edge satisfying properties (i)-(iv). So we just need to worry about elements of $\mathrm{S}\left(\widetilde{\mathfrak{D}}_{k}\right) \backslash \widetilde{\mathfrak{D}}_{k}$, all of which are rays.

Given a ray $\mathfrak{d} \in \widetilde{\mathfrak{D}}_{k}^{\infty}$, define the graph $\Gamma_{\mathfrak{d}}$ by

$$
\begin{aligned}
& \Gamma_{\mathfrak{d}}^{[0]}=\left\{\mathfrak{d}^{\prime} \mid \mathfrak{d}^{\prime} \in \operatorname{Ancestors}(\mathfrak{d}) \text { and } \mathfrak{d}^{\prime} \text { a ray }\right\} \\
& \Gamma_{\mathfrak{d}}^{[1]}=\operatorname{Ancestors}(\mathfrak{d})
\end{aligned}
$$

as abstract sets, writing the edge corresponding to $\mathfrak{d}^{\prime}$ as $E_{\mathfrak{d}^{\prime}}$ and the vertex $V_{\mathfrak{d}^{\prime}}$. If $\mathfrak{d}^{\prime} \in \operatorname{Ancestors}(\mathfrak{d}) \backslash\{\mathfrak{d}\}$, then $\mathfrak{d}^{\prime}$ is parent to a unique ray in Ancestors $(\mathfrak{d})$, which we write as $\operatorname{Child}\left(\mathfrak{d}^{\prime}\right)$. If $\mathfrak{d}$ is itself a line, then we are in a degenerate case, and $\Gamma_{\mathfrak{o}}$ is just a line (an edge with no vertices). If $\mathfrak{d}$ is a ray, then to define $\Gamma_{\mathfrak{d}}$, we specify that for $\mathfrak{d}^{\prime} \in \operatorname{Ancestors}(\mathfrak{d})$, either

- $\mathfrak{d}^{\prime} \neq \mathfrak{d}$ and $\mathfrak{d}^{\prime}$ is a ray, in which case the vertices of $E_{\mathfrak{d}^{\prime}}$ are $V_{\mathfrak{d}^{\prime}}$ and $V_{\text {Child( }\left(\mathfrak{d}^{\prime}\right)}$.

- $\mathfrak{d}^{\prime}=\mathfrak{d}$, in which case $E_{\mathfrak{d}^{\prime}}$ is an unbounded edge with vertex $V_{\mathfrak{d}}$.

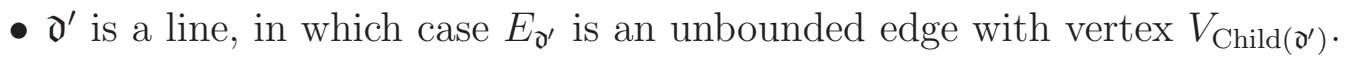

We next define the weight function $w_{\Gamma_{\mathfrak{d}}}$ as follows. For any $\mathfrak{d}^{\prime} \in \operatorname{Ancestors}(\mathfrak{d}), f_{\mathfrak{d}^{\prime}}$ takes the form $1+c_{\mathfrak{d}^{\prime}} z^{m_{\mathfrak{d}^{\prime}}}$ for some $c_{\mathfrak{d}^{\prime}} \in \tilde{R}_{k}$ and $m_{\mathfrak{d}^{\prime}} \in M \backslash\{0\}$. We define $w_{\Gamma_{\mathfrak{o}}}\left(E_{\mathfrak{d}^{\prime}}\right)$ to be the index of $m_{\mathfrak{d}^{\prime}}$, with $m_{\mathfrak{d}^{\prime}}^{\prime}$ given by

$$
m_{\mathfrak{d}^{\prime}}=w_{\Gamma_{\mathfrak{d}}}\left(E_{\mathfrak{d}^{\prime}}\right) m_{\mathfrak{d}^{\prime}}^{\prime} .
$$


Finally, we define $h$ in the obvious way, mapping $E_{\mathfrak{d}^{\prime}}$ in the respective cases to the line segment joining $\operatorname{Init}\left(\mathfrak{d}^{\prime}\right)$ and $\operatorname{Init}\left(\operatorname{Child}\left(\mathfrak{d}^{\prime}\right)\right), \mathfrak{d}$, and the ray $\mathbb{R}_{\leq 0} m_{\mathfrak{d}^{\prime}}+\operatorname{Init}\left(\operatorname{Child}\left(\mathfrak{d}^{\prime}\right)\right)$.

Since $\Gamma$ is a tree, the genus 0 condition is clearly satisfied. We need to check the balancing condition at every vertex $V=V_{\mathfrak{d}^{\prime}}$. Let Parents $\left(\mathfrak{d}^{\prime}\right)=\left\{\mathfrak{d}_{1}, \mathfrak{d}_{2}\right\}$. From (1.4), we see

$$
m_{\mathfrak{d}^{\prime}}=m_{\mathfrak{d}_{1}}+m_{\mathfrak{d}_{2}}
$$

or equivalently

$$
w_{\Gamma_{\mathfrak{d}}}\left(E_{\mathfrak{d}^{\prime}}\right) m_{\mathfrak{d}^{\prime}}^{\prime}=w_{\Gamma_{\mathfrak{d}}}\left(E_{\mathfrak{d}_{1}}\right) m_{\mathfrak{d}_{1}}^{\prime}+w_{\Gamma_{\mathfrak{d}}}\left(E_{\mathfrak{d}_{2}}\right) m_{\mathfrak{d}_{2}}^{\prime},
$$

which is the balancing condition at $V$, keeping in mind that $m_{\mathfrak{d}_{1}}^{\prime}$ and $m_{\mathfrak{d}_{2}}^{\prime}$ point towards $V$ and $m_{\mathfrak{d}^{\prime}}^{\prime}$ points away from $V$. By the generality condition on $\widetilde{\mathfrak{D}}_{k}, \Gamma_{\mathfrak{d}}$ has at most trivalent vertices.

Next, we check the expression given for $f_{\mathfrak{d}}$ inductively. If $\mathfrak{d}$ is a line, then $\Gamma$ is just a line, and $\operatorname{Mult}(h)=1$ since there are no trivalent vertices whatsoever. Formula (2.1) is correct by the definition of the original deformed scattering diagram $\widetilde{\mathfrak{D}}_{k}$ in Section 1.4

Suppose $\mathfrak{d}$ is a ray and (2.1) holds for both Parents $(\mathfrak{d})=\left\{\mathfrak{d}_{1}, \mathfrak{d}_{2}\right\}$. Let $h_{1}$ and $h_{2}$ be the tropical curves corresponding to the respective parents. By (1.4),

$$
\begin{aligned}
& f_{\mathfrak{d}}=1+w_{\Gamma_{\mathfrak{d}}}\left(E_{\mathfrak{d}_{1}}\right) \operatorname{Mult}\left(h_{1}\right) w_{\Gamma_{\mathfrak{d}}}\left(E_{\mathfrak{d}_{2}}\right) \operatorname{Mult}\left(h_{2}\right) \\
& \cdot w_{\text {out }}\left|m_{\mathfrak{d}_{1}}^{\prime} \wedge m_{\mathfrak{d}_{2}}^{\prime}\right| \prod_{i, J, w}\left((\# J) ! a_{i(\# J) w} \prod_{j \in J} u_{i j}\right) z^{m_{\text {out }}}
\end{aligned}
$$

where the first product is over all indices $i, J, w$ for which $\mathfrak{d}_{i J w} \in \operatorname{Leaves}(\mathfrak{d})$. The coefficient in front of the product is just

$$
\operatorname{Mult}\left(h_{1}\right) \operatorname{Mult}\left(h_{2}\right) \operatorname{Mult}_{V_{\mathfrak{d}}}(h) w_{\text {out }}=\operatorname{Mult}(h) w_{\text {out }} .
$$

The derivation of (2.1) is complete.

Finally, by unravelling the definitions, each rational tropical curve satisfying conditions (i)-(iv) is easily seen to correspond to a unique element of $\widetilde{\mathfrak{D}}_{k}^{\infty}$.

As we shall see, Theorem 2.4 implies that the scattering diagrams are computing a tropical enumerative invariant. We shall relate the tropical enumerations which arise first to holomorphic enumerations and then to relative Gromov-Witten invariants.

Example 2.5. Returning to the situation of Example 1.11, consider the ray of slope 1 which is the third one from the upper left in Figure 1.3. Figure 2.1 shows the corresponding tropical curve with the outgoing edge of weight two labelled. The multiplicity of the curve is 2 , and the function attached to the outgoing ray is $1+4 u_{11} u_{12} u_{21} u_{22} x^{2} y^{2}$. 


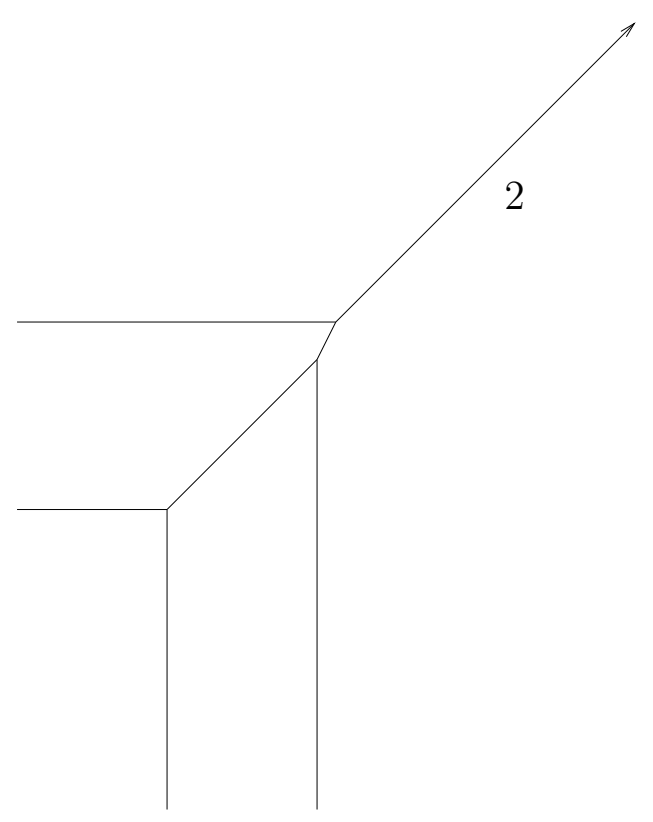

FigURE 2.1.

2.3. Tropical counts. Let $m_{1}, \ldots, m_{n} \in M$ be primitive elements, and let

$$
\mathbf{m}=\left(m_{1}, \ldots, m_{n}\right)
$$

denote the $n$-tuple. Let the lines

$$
\mathfrak{d}_{i j}=m_{i j}+\mathbb{R} m_{i}, \quad m_{i j} \in M_{\mathbb{R}}
$$

be chosen generally for $1 \leq i \leq n$ and $1 \leq j \leq l_{i}$.

Let $\mathbf{w}_{i}=\left(w_{i 1}, \ldots, w_{i l_{i}}\right)$ be weight vectors with

$$
0<w_{i 1} \leq w_{i 2} \leq \cdots \leq w_{i l_{i}}, \quad w_{i j} \in \mathbb{Z} .
$$

The weight vector $\mathbf{w}_{i}$ has length $l_{i}$ and size

$$
\left|\mathbf{w}_{i}\right|=\sum_{j=1}^{l_{i}} w_{i j} .
$$

Denote the $n$-tuple of weight vectors by $\mathbf{w}=\left(\mathbf{w}_{1}, \ldots, \mathbf{w}_{n}\right)$. We only consider weight vectors for which

$$
m_{\mathrm{out}}=\sum_{i=1}^{n}\left|\mathbf{w}_{i}\right| m_{i} \neq 0 .
$$

Definition 2.6. Let $N_{\mathbf{m}}^{\text {trop }}(\mathbf{w})$ be the number of rational tropical curves $h: \Gamma \rightarrow M_{\mathbb{R}}$ counted with the multiplicity of Definition 2.2 where

$$
\Gamma_{\infty}^{[1]}=\left\{E_{i j} \mid 1 \leq i \leq n, 1 \leq j \leq l_{i}\right\} \cup\left\{E_{\text {out }}\right\}
$$


with

- $h\left(E_{i j}\right) \subseteq \mathfrak{d}_{i j}$ and $-m_{i}$ pointing in the unbounded direction of $h\left(E_{i j}\right)$,

- $w_{\Gamma}\left(E_{i j}\right)=w_{i j}$,

- $h\left(E_{\text {out }}\right)$ pointing in the direction of $m_{\text {out }}$.

Note that $w_{\Gamma}\left(E_{\text {out }}\right)$, the weight of $E_{\text {out }}$, coincides with the index of $m_{\text {out }}$

Proposition 2.7. The numbers $N_{\mathbf{m}}^{\mathrm{trop}}(\mathbf{w})$ do not depend on the (generic) choice of the vectors $m_{i j}$.

Proof. The result follows from standard tropical arguments - for example the method of [6] suffices. Alternatively, we will later show that these tropical invariants agree with holomorphic counts which are independent of any choices.

Let $\mathfrak{D}=\left\{\left(\mathfrak{d}_{i}, f_{i}\right) \mid 1 \leq i \leq n\right\}$ be a standard scattering diagram over the ring $\mathbb{C}\left[\left[t_{1}, \ldots, t_{n}\right]\right]$ consisting of a number of lines through the origin. Let $\mathfrak{d}_{i}=\mathbb{R} m_{i}$ with $m_{i}$ primitive. Since $t_{i}$ is the only power series variable occurring in $f_{i}$, we can write the logarithm as

$$
\log f_{i}=\sum_{j=1}^{\infty} \sum_{w \geq 1} w a_{i j w} z^{w m_{i}} t_{i}^{j}
$$

with $a_{i j w} \in \mathbb{C}$.

Let $\mathbf{S}(\mathfrak{D})$ be the associated scattering diagram. We assume $\mathbf{S}(\mathfrak{D})$ has at most one ray in any given direction.

Theorem 2.8. Let $\left(\mathfrak{d}, f_{\mathfrak{d}}\right) \in \mathrm{S}(\mathfrak{D}) \backslash \mathfrak{D}$ be a ray. Then,

$$
\log f_{\mathfrak{o}}=\sum_{\mathbf{w}} \sum_{\mathbf{k}} \frac{w_{\text {out }}(\mathbf{w}) N_{\mathbf{m}}^{\mathrm{trop}}(\mathbf{w})}{|\operatorname{Aut}(\mathbf{w}, \mathbf{k})|}\left(\prod_{\substack{1 \leq i \leq n \\ 1 \leq j \leq l_{i}}} a_{i k_{i j} w_{i j}} t_{i}^{k_{i j}}\right) z^{\sum_{i}\left|\mathbf{w}_{i}\right| m_{i}}
$$

where

- The first sum is over all $n$-tuples of weight vectors $\mathbf{w}=\left(\mathbf{w}_{1}, \ldots, \mathbf{w}_{n}\right)$ satisfying $0 \neq \sum_{i}\left|\mathbf{w}_{i}\right| m_{i} \in \mathfrak{d}$. Let $l_{i}=\operatorname{length}\left(\mathbf{w}_{i}\right)$.

- The second sum is over all $n$-tuples of vectors $\mathbf{k}=\left(\mathbf{k}_{1}, \ldots, \mathbf{k}_{n}\right)$ where

$$
\mathbf{k}_{i}=\left(k_{i 1}, \ldots, k_{i l_{i}}\right)
$$

for positive integers $k_{i j}$ satisfying

$$
k_{i j} \leq k_{i(j+1)} \quad \text { if } \quad w_{i j}=w_{i(j+1)} .
$$

Note length $\left(\mathbf{k}_{i}\right)=$ length $\left(\mathbf{w}_{i}\right)$.

- $\sum_{i}\left|\mathbf{w}_{i}\right| m_{i}=w_{\text {out }}(\mathbf{w}) m_{\text {out }}^{\prime}$ with $m_{\text {out }}^{\prime} \in M$ primitive. 
- $\operatorname{Aut}\left(\mathbf{w}_{i}, \mathbf{k}_{i}\right)$ denotes the subgroup of the permutation group $\Sigma_{l_{i}}$ stabilizing the data

$$
\left(\left(w_{i 1}, k_{i 1}\right), \ldots,\left(w_{i l_{i}}, k_{i l_{i}}\right)\right)
$$

$\left|\operatorname{Aut}\left(\mathbf{w}_{i}, \mathbf{k}_{i}\right)\right|$ denotes the order, and

$$
|\operatorname{Aut}(\mathbf{w}, \mathbf{k})|=\prod_{i=1}^{n}\left|\operatorname{Aut}\left(\mathbf{w}_{i}, \mathbf{k}_{i}\right)\right| .
$$

Proof. We will prove (2.2) modulo the ideal $I_{k}=\left(t_{1}^{k+1}, \ldots, t_{n}^{k+1}\right)$ and let $k \rightarrow \infty$. Modulo $I_{k}$, the diagram $\mathrm{S}(\mathfrak{D})$ is equivalent to $\left(\widetilde{\mathfrak{D}}_{k}^{\infty}\right)_{\text {as }}$. Thus modulo $I_{k}$,

$$
\log f_{\mathfrak{d}}=\sum_{\mathfrak{d}^{\prime}} \log f_{\mathfrak{d}^{\prime}}
$$

where the sum is over rays $\mathfrak{d}^{\prime} \in \widetilde{\mathfrak{D}}_{k}^{\infty}$ parallel to $\mathfrak{d}$.

Theorem 2.4 gives a correspondence between such rays and tropical curves

$$
h: \Gamma \rightarrow M_{\mathbb{R}}
$$

Consider one such ray $\mathfrak{d}^{\prime}$ and the corresponding tropical curve $h: \Gamma \rightarrow M_{\mathbb{R}}$. Then, there are weight vectors $\mathbf{w}_{1}(h), \ldots, \mathbf{w}_{n}(h)$ of lengths $l_{1}, \ldots, l_{n}$, and sets

$$
J_{i j}(h) \subseteq\{1, \ldots, k\}, \quad 1 \leq j \leq l_{i}
$$

with $J_{i 1}(h), \ldots, J_{i l_{i}}(h)$ pairwise disjoint, such that we can write

$$
\operatorname{Leaves}\left(\mathfrak{d}^{\prime}\right)=\left\{\mathfrak{d}_{i J_{i j}(h) w_{i j}(h)} \mid 1 \leq i \leq n, 1 \leq j \leq l_{i}\right\}
$$

and

$$
\Gamma_{\infty}^{[1]}=\left\{E_{i j} \mid 1 \leq i \leq n, 1 \leq j \leq l_{i}\right\} \cup\left\{E_{\text {out }}\right\}
$$

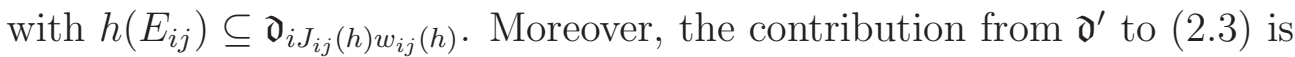

$$
\log f_{\mathfrak{d}^{\prime}}=w_{\Gamma}\left(E_{\text {out }}\right) \operatorname{Mult}(h) \prod_{\substack{1 \leq i \leq n \\ 1 \leq j \leq l_{i}}}\left(\left(\# J_{i j}(h)\right) ! a_{i\left(\# J_{i j}(h)\right) w_{i j}(h)} \prod_{l \in J_{i j}(h)} u_{i l}\right) z^{\sum_{i}\left|\mathbf{w}_{i}(h)\right| m_{i}} .
$$

The contribution from all tropical curves $h$ giving rise to the same weight vectors $\mathbf{w}_{i}$ and the same sets $J_{i j}$ with $k_{i j}=\# J_{i j}$ defining vectors $\mathbf{k}_{1}, \ldots, \mathbf{k}_{n}$, is then

$$
w_{\text {out }}(\mathbf{w}) N_{\mathbf{m}}^{\text {trop }}(\mathbf{w}) \prod_{\substack{1 \leq i \leq n \\ 1 \leq j \leq l_{i}}}\left(k_{i j} ! a_{i k_{i j} w_{i j}} \prod_{l \in J_{i j}} u_{i l}\right) z^{\sum_{i}\left|\mathbf{w}_{i}\right| m_{i}} .
$$

We first keep both $\mathbf{w}$ and $\mathbf{k}$ fixed and choose sets $J_{1}, \ldots, J_{n} \subseteq\{1, \ldots, k\}$ with $\# J_{i}=\left|\mathbf{k}_{i}\right|$. We then sum over all possible ways of writing $J_{i}$ as a disjoint union of sets $J_{i j}$ with $\# J_{i j}=k_{i j}$. There are $\left|\mathbf{k}_{i}\right| ! / \prod_{j} k_{i j}$ ! ways of writing $J_{i}$ as such a disjoint union. However, we have overcounted curves. If $\sigma \in \operatorname{Aut}\left(\mathbf{w}_{i}, \mathbf{k}_{i}\right)$, we have

$$
\left\{\left(i, J_{i j}, w_{i j}\right) \mid 1 \leq j \leq \operatorname{length}\left(\mathbf{w}_{i}\right)\right\}=\left\{\left(i, J_{i \sigma(j)}, w_{i \sigma(j)}\right) \mid 1 \leq j \leq \operatorname{length}\left(\mathbf{w}_{i}\right)\right\} .
$$


Thus the contribution from a choice of the sets $J_{i}$ is

$$
\frac{w_{\text {out }}(\mathbf{w}) N_{\mathbf{m}}^{\text {trop }}(\mathbf{w})}{|\operatorname{Aut}(\mathbf{w}, \mathbf{k})|}\left(\prod_{1 \leq i \leq n}\left(\prod_{1 \leq j \leq l_{i}} k_{i j} ! a_{i k_{i j} w_{i j}}\right) \frac{\left|\mathbf{k}_{i}\right| !}{\prod_{j} k_{i j} !} \prod_{l \in J_{i}} u_{i l}\right) z^{\sum_{i}\left|\mathbf{w}_{i}\right| m_{i}} .
$$

Summing over possible $J_{i}$ 's, we get

$$
\frac{w_{\text {out }}(\mathbf{w}) N_{\mathbf{m}}^{\mathrm{trop}}(\mathbf{w})}{|\operatorname{Aut}(\mathbf{w}, \mathbf{k})|}\left(\prod_{1 \leq i \leq n}\left(\prod_{1 \leq j \leq l_{i}} a_{i k_{i j} w_{i j}}\right) \sum_{\substack{J_{i} \\ \# J_{i}=\left|\mathbf{k}_{i}\right|}}\left|\mathbf{k}_{i}\right| ! \prod_{l \in J_{i}} u_{i l}\right) z^{\sum_{i}\left|\mathbf{w}_{i}\right| m_{i}}
$$

But

$$
\begin{aligned}
t_{i}^{\left|\mathbf{k}_{i}\right|} & =\left(u_{1}+\cdots+u_{k}\right)^{\left|\mathbf{k}_{i}\right|} \\
& =\left|\mathbf{k}_{i}\right| ! \sum_{\substack{J_{i} \\
\# J_{i}=\left|\mathbf{k}_{i}\right|}} \prod_{l \in J_{i}} u_{i l}
\end{aligned}
$$

from which we find the total contribution from curves with given $\mathbf{w}_{i}$ and $\mathbf{k}_{i}$ is

$$
\frac{w_{\text {out }}(\mathbf{w}) N_{\mathbf{m}}^{\operatorname{trop}}(\mathbf{w})}{|\operatorname{Aut}(\mathbf{w}, \mathbf{k})|}\left(\prod_{\substack{1 \leq i \leq n \\ 1 \leq j \leq l_{i}}} a_{i k_{i j} w_{i j}} t_{i}^{k_{i j}}\right) z^{\sum_{i}\left|\mathbf{w}_{i}\right| m_{i}}
$$

giving the desired result.

\section{From tropical to holomorphic COUNTS}

3.1. Holomorphic counts. We now describe the holomorphic analogue of $N_{\mathbf{m}}^{\operatorname{trop}}(\mathbf{w})$. Following the notation of Section 2.3, let

$$
\mathbf{m}=\left(m_{1}, \ldots, m_{n}\right)
$$

be an $n$-tuple of primitive vectors of $M$. Let $\mathbf{w}=\left(\mathbf{w}_{1}, \ldots, \mathbf{w}_{n}\right)$ be an $n$-tuple of weight vectors

$$
\mathbf{w}_{i}=\left(w_{i 1}, \ldots, w_{i l_{i}}\right)
$$

with

$$
0 \neq m_{\text {out }}=\sum_{i=1}^{n}\left|\mathbf{w}_{i}\right| m_{i}, \quad m_{\text {out }}=w_{\text {out }} m_{\text {out }}^{\prime}
$$

for $m_{\text {out }}^{\prime} \in M$ primitive.

To match the conventions set for standard scattering diagrams in Definition 1.10, we do not require the $m_{1}, \ldots, m_{n}$ to be distinct. However, we will only treat the distinct case since the multiplicities here will not matter (and complicate the notation). A more subtle issue concerns $m_{\text {out }}$. Either the ray generated by $m_{\text {out }}$ is distinct from the rays generated by $-m_{1}, \ldots,-m_{n}$ or not. We will present a treatment of the former case. The discussion in the degenerate case where the ray generated by $m_{\text {out }}$ coincides with 
a ray generated by $-m_{k}$ is almost identical. We state the results there and leave the details to the reader.

So we assume the ray generated by $m_{\text {out }}$ is distinct from the rays generated by the $-m_{i}$. Let $\Sigma$ denote the complete rational fan in $M_{\mathbb{R}}$ whose rays are generated by

$$
-m_{1}, \ldots,-m_{n}, m_{\text {out }}
$$

Let $X$ denote the corresponding toric surface over $\mathbb{C}$. Let

$$
D_{1}, \ldots, D_{n}, D_{\text {out }} \subseteq X
$$

be the toric divisors corresponding to the given rays. Let $X^{o}$ be the complement of the 0-dimensional torus orbits in $X$, and let

$$
D_{i}^{o}=D_{i} \cap X^{o}, \quad D_{\text {out }}^{o}=D_{\text {out }} \cap X^{o} .
$$

Let $\beta_{\mathbf{w}} \in H_{2}(X, \mathbb{Z})$ be the homology class defined by the conditions

$$
D_{i} \cdot \beta_{\mathbf{w}}=\left|\mathbf{w}_{i}\right|, \quad D_{\text {out }} \cdot \beta_{\mathbf{w}}=w_{\text {out }} .
$$

Define the open subspace

$$
\mathfrak{M}\left(X^{o}, \mathbf{w}\right) \subseteq \overline{\mathfrak{M}}_{0,1+\sum_{i=1}^{n} l_{i}}\left(X, \beta_{\mathbf{w}}\right)
$$

of the moduli space of genus 0 stable maps represented by maps of the form

$$
\varphi:\left(\mathbb{P}^{1}, Q_{11}, \ldots, Q_{1 l_{1}}, \ldots, Q_{n 1}, \ldots, Q_{n l_{n}}, Q\right) \rightarrow X
$$

satisfying the following properties:

(i) $\varphi\left(Q_{i j}\right) \in D_{i}^{o}$ and $\varphi^{*}\left(t_{i}\right)$ has a zero of order $w_{i j}$ at $Q_{i j}$ for a local defining equation $t_{i}$ for $D_{i}$.

(ii) $\varphi(Q) \in D_{\text {out }}^{o}$ and $\varphi^{*}\left(t_{\text {out }}\right)$ has a zero of order $w_{\text {out }}$ at $Q$ for a local defining equation $t_{\text {out }}$ for $D_{\text {out }}$.

Since all the intersections of $\varphi$ with the toric divisors of $X$ are accounted for, there is a factorization

$$
\varphi: \mathbb{P}^{1} \rightarrow X^{o} \subseteq X
$$

We may equivalently view $\mathfrak{M}\left(X^{o}, \mathbf{w}\right)$ as an open subspace of the moduli space of genus 0 stable maps to $X^{o}$ of class $\beta_{\mathbf{w}}$ relative to the divisors $D_{1}^{o}, \ldots, D_{n}^{o}, D_{\text {out }}$ with relative conditions specified by $\mathbf{w}$ and $w_{\text {out }}$. The relative perspective will be pursued in Sections 4 and 5 .

Definition 3.1. There is an evaluation map

$$
\mathrm{ev}: \mathfrak{M}\left(X^{o}, \mathbf{w}\right) \rightarrow \prod_{i=1}^{n}\left(D_{i}^{o}\right)^{l_{i}}
$$


If the evaluation map is generically finite, then we define $N_{\mathbf{m}}^{\text {hol }}(\mathbf{w})$ to be the degree. Otherwise we define $N_{\mathbf{m}}^{\text {hol }}(\mathbf{w})$ to be 0 .

The count $N_{\mathbf{m}}^{\mathrm{hol}}(\mathbf{w})$ is invariant under refinement of the fan $\Sigma$. The curves which appear in $\mathfrak{M}\left(X^{o}, \mathbf{w}\right)$ are disjoint from the 0-dimensional torus orbits of $X$, and hence lift to any toric blow-up of $X$. Conversely, any curve on a toric blow-up of $X$ disjoint from the exceptional divisors is the lifting of a curve on $X$. As a consequence, we can always assume that $X$ is nonsingular by adding additional vectors $m_{i}$ with associated weight vectors of length 0 .

By the following result, the dimensions of the domain and the target of the evaluation map (3.1) coincide.

Proposition 3.2. $\mathfrak{M}\left(X^{o}, \mathbf{w}\right)$ is a nonsingular Deligne-Mumford stack of dimension $\sum_{i=1}^{n} \operatorname{length}\left(\mathbf{w}_{i}\right)$.

Proof. We first review the standard quotient presentation of $X$, which we assume to be non-singular. Let $T$ be the free abelian group generated by $\Sigma^{[1]}$, the set of rays of $\Sigma$. Let

$$
T_{\mathbb{C}}=T \otimes_{\mathbb{Z}} \mathbb{C}
$$

We may view $T_{\mathbb{C}}$ as an affine space,

$$
T_{\mathbb{C}}=\operatorname{Spec} \mathbb{C}\left[\left\{z^{\rho} \mid \rho \in \Sigma^{[1]}\right\}\right] .
$$

Given a subset $S \subseteq \Sigma^{[1]}$, define $\mathbf{A}(S)$ to be the subspace of $T_{\mathbb{C}}$ determined by the equations $\left\{z^{\rho}=0 \mid \rho \in S\right\}$. Set

$$
Z=\bigcup_{S} \mathbf{A}(S)
$$

where the union is over all subsets $S \subseteq \Sigma^{[1]}$ which are not the rays of some cone in $\Sigma$. Set

$$
U=T_{\mathbb{C}} \backslash Z
$$

There is a natural map $r: T \rightarrow M$ taking a generator $\rho \in \Sigma^{[1]}$ of $T$ to $m_{\rho} \in M$, the primitive generator of the ray $M$. This yields an exact sequence

$$
0 \longrightarrow K \longrightarrow T \stackrel{r}{\longrightarrow} M \longrightarrow 0 .
$$

The algebraic torus $K \otimes_{\mathbb{Z}} \mathbb{C}^{*}$ acts on $T_{\mathbb{C}}$, and we have

$$
X=U / K \otimes_{\mathbb{Z}} \mathbb{C}^{*}
$$

Because $X$ is nonsingular, the $K \otimes_{\mathbb{Z}} \mathbb{C}^{*}$ action on $U$ is free.

We write the coordinates on $T_{\mathbb{C}}$ as $z_{1}, \ldots, z_{n}, z_{\text {out }}$ corresponding to the rays generated by $-m_{1}, \ldots,-m_{n}$ and $m_{\text {out }}$. These are our homogeneous coordinates on $X$. The divisor $D_{i}$ is given by $z_{i}=0$ and the divisor $D_{\text {out }}$ is given by $z_{\text {out }}=0$. 
A parameterized map $\varphi: \mathbb{P}^{1} \rightarrow X$ with image of class $\beta$ yields (up to an action of $\left.K \otimes \mathbb{C}^{*}\right)$ homogeneous polynomials $\varphi_{1}, \ldots, \varphi_{n}, \varphi_{\text {out }}$ in the coordinate ring $\mathbb{C}[u, v]$ of $\mathbb{P}^{1}$ with $\operatorname{deg} \varphi_{i}=\left|\mathbf{w}_{i}\right|$ which avoid $Z$. Conversely, such polynomials $\varphi=\left(\varphi_{1}, \ldots, \varphi_{n}, \varphi_{\text {out }}\right)$ determine a unique parameterized map.

If $\varphi \in \mathfrak{M}\left(X^{o}, \mathbf{w}\right)$, we can always apply an element of $\mathrm{PGL}\left(\mathbb{P}^{1}\right)$ so that

$$
Q=(1: 0) \in \mathbb{P}^{1} \text {. }
$$

Then, we must have

(i) $\varphi_{i}=c_{i} \prod_{j=1}^{l_{i}}\left(u-a_{i j} v\right)^{w_{i j}}$ for some $c_{i} \in \mathbb{C}^{*}, a_{i j} \in \mathbb{C}$.

(ii) $\varphi_{\text {out }}=c_{\text {out }} v^{w_{\text {out }}}, c_{\text {out }} \in \mathbb{C}^{*}$.

Furthermore, such a choice of $\varphi_{i}, \varphi_{\text {out }}$ gives rise to $\varphi \in \mathfrak{M}\left(X^{o}, \mathbf{w}\right)$ if and only if all the $a_{i j}$ 's are distinct. The space of choices of $\varphi_{i}, \varphi_{\text {out }}$ is therefore an open subset of

$$
\left(\mathbb{C}^{*}\right)^{n+1} \times \prod_{i=1}^{n} \mathbb{C}^{\text {length }\left(\mathbf{w}_{i}\right)} .
$$

We then have to divide out by the action of $K \otimes \mathbb{C}^{*}$, which is of dimension $(n+1)-2$, as well as the 2-dimensional subgroup of $\operatorname{PGL}\left(\mathbb{P}^{1}\right)$ keeping (1:0) fixed (to remove the parameterization). Since these actions have at most finite stabilizers, we obtain a nonsingular Deligne-Mumford stack of dimension $\sum_{i=1}^{n} \operatorname{length}\left(\mathbf{w}_{i}\right)$.

We will need later a dimension bound for the same toric geometry $X^{o}$, but with different curve classes. Let $\beta_{\widehat{\mathbf{w}}} \in H_{2}\left(X^{o}, \mathbb{Z}\right)$ be a class defined by the conditions

$$
D_{i} \cdot \beta_{\widehat{\mathbf{w}}}=\left|\widehat{\mathbf{w}}_{i}\right|, \quad D_{\text {out }} \cdot \beta_{\widehat{\mathbf{w}}}=0
$$

for weight vectors $\widehat{\mathbf{w}}=\left(\widehat{\mathbf{w}}_{1}, \ldots \widehat{\mathbf{w}}_{n}\right)$ satisfying

$$
\sum_{i=1}^{n}\left|\widehat{\mathbf{w}}_{i}\right| m_{i}=0 .
$$

Then, the proof of Proposition 3.2 immediately yields the following result.

Proposition 3.3. $\mathfrak{M}\left(X^{o}, \widehat{\mathbf{w}}\right)$ is a nonsingular Deligne-Mumford stack of dimension $-1+\sum_{i=1}^{n} \operatorname{length}\left(\widehat{\mathbf{w}}_{i}\right)$.

3.2. Equivalence. The following result relating the holomorphic and tropical counts will play a crucial role for us.

Theorem 3.4. $N_{\mathbf{m}}^{\mathrm{trop}}(\mathbf{w})=N_{\mathbf{m}}^{\mathrm{hol}}(\mathbf{w}) \cdot \prod_{i=1}^{n} \prod_{j=1}^{l_{i}} w_{i j}$.

Tropical counting of holomorphic curves on toric varieties with incidence conditions occurs in [19, 20]. The latter reference already includes higher order tangency conditions with the toric boundary. The only difference between [20] and the present 
situation is the treatment of point conditions - here we impose point conditions on the toric boundary instead of in the big cell. Very little has be to added to [20] to obtain Theorem 3.4. The required modifications are straightforward. A more detailed discussion is presented in the Appendix.

3.3. Degenerate case. Suppose the ray generated by $m_{\text {out }}$ coincides with the ray generated by $-m_{k}$. Let $\Sigma$ denote the complete rational fan in $M_{\mathbb{R}}$ whose rays are generated by

$$
-m_{1}, \ldots,-m_{n}
$$

Let $X$ denote the corresponding toric surface over $\mathbb{C}$. Let

$$
D_{1}, \ldots, D_{n} \subseteq X
$$

be the toric divisors corresponding to the given rays and let $D_{\text {out }}=D_{k}$.

Let $\beta_{\mathbf{w}} \in H_{2}(X, \mathbb{Z})$ be the homology class defined by the conditions

$$
D_{i} \cdot \beta_{\mathbf{w}}=\left|\mathbf{w}_{i}\right| \text { for } i \neq k, \quad D_{k} \cdot \beta_{\mathbf{w}}=\left|\mathbf{w}_{k}\right|+w_{\text {out }} .
$$

We define moduli spaces $\mathfrak{M}\left(X^{o}, \mathbf{w}\right)$ of maps of class $\beta_{\mathbf{w}}$, evaluation maps, and invariants $N_{\mathbf{m}}^{\mathrm{hol}}(\mathbf{w})$ in exactly the same manner as before. The only difference is the contact point $Q$ of

$$
\varphi:\left(\mathbb{P}^{1}, Q_{11}, \ldots, Q_{1 l_{1}}, \ldots, Q_{n 1}, \ldots, Q_{n l_{n}}, Q\right) \rightarrow X
$$

shares a divisor with $Q_{k 1}, \ldots, Q_{k l_{k}}$. The rest is straightforward. All the parallel results hold including Theorem 3.4 .

\section{Gromov-Witten theory}

4.1. Overview. We will now connect the holomorphic counts $N_{\mathbf{m}}^{\text {hol }}(\mathbf{w})$ defined in Definition 3.1 to Gromov-Witten theory. Usually, the latter subject is studied for compact geometries. For us, the open target consisting of $X^{o}$ relative to the disjoint divisors $D_{1}^{o}, \ldots, D_{n}^{o}, D_{\text {out }}^{o}$ is much more natural. In order to define relative invariants for our open geometries, properness of the associated evaluations maps will be proven. We will obtain another equivalence

$$
N_{\mathbf{m}}^{\mathrm{hol}}(\mathbf{w})=N_{\mathbf{m}}^{\mathrm{rel}}(\mathbf{w})
$$

where the term on the right is a relative Gromov-Witten count for $X^{o}$.

In fact, Gromov-Witten theory provides a direct interpretation of the entire summation of Theorem 2.8. We will find $\log f_{\mathfrak{d}}$ is simply a summation of genus 0 GromovWitten invariants of blow-ups of $X^{o}$ relative to $D_{\text {out }}^{o}$. The result will be proven in Section 5 from Theorem 2.8 by the equivalences, the degeneration formula, and exact multiple cover calculations in Gromov-Witten theory. 
4.2. Desingularization. Following the notation of Section [2.3, let

$$
\mathbf{m}=\left(m_{1}, \ldots, m_{n}\right)
$$

be an $n$-tuple of primitive vectors of $M$. Let $\mathbf{w}=\left(\mathbf{w}_{1}, \ldots, \mathbf{w}_{n}\right)$ be a $n$-tuple of weight vectors

$$
\mathbf{w}_{i}=\left(w_{i 1}, \ldots, w_{i l_{i}}\right)
$$

with

$$
0 \neq m_{\text {out }}=\sum_{i=1}^{n}\left|\mathbf{w}_{i}\right| m_{i}, \quad m_{\text {out }}=w_{\text {out }} m_{\text {out }}^{\prime}
$$

for $m_{\text {out }}^{\prime} \in M$ primitive. We again treat the nondegenerate case where the ray generated by $m_{\text {out }}$ is distinct from the rays generated by $-m_{i}$.

Let $\Sigma$ be the fan with rays generated by

$$
-m_{1}, \ldots,-m_{n}, m_{\text {out }} \text {. }
$$

Let $X$ be the toric surface over $\mathbb{C}$ associated to $\Sigma$ with toric divisors $D_{1}, \ldots, D_{n}, D_{\text {out }}$. Let $\widetilde{\Sigma}$ be a refinement of $\Sigma$ satisfying the following two properties:

(i) the toric surface $\widetilde{X}$ associated to $\widetilde{\Sigma}$ is nonsingular,

(ii) the proper transforms of $D_{1}, \ldots, D_{n}, D_{\text {out }}$ under the birational toric morphism $\widetilde{X} \rightarrow X$ are pairwise disjoint.

Let $\widetilde{D}_{1}, \ldots, \widetilde{D}_{n}, \widetilde{D}_{\text {out }} \subseteq \widetilde{X}$ denote the respective proper transforms of the divisors.

Let $\beta_{\mathbf{w}} \in H_{2}(\widetilde{X}, \mathbb{Z})$ be as in 93 . The class $\beta_{\mathbf{w}}$ is determined by intersection numbers with toric divisors,

$$
\widetilde{D}_{i} \cdot \beta_{\mathbf{w}}=\left|\mathbf{w}_{i}\right|, \quad \widetilde{D}_{\text {out }} \cdot \beta_{\mathbf{w}}=w_{\text {out }}
$$

and $D \cdot \beta_{\mathbf{w}}=0$ if $D \notin\left\{\widetilde{D}_{1}, \ldots, \widetilde{D}_{n}, \widetilde{D}_{\text {out }}\right\}$. The full toric boundary $B \subseteq \widetilde{X}$ represents the anticanonical class of $\widetilde{X}$. Hence

$$
\int_{\beta_{\mathbf{w}}} c_{1}\left(T_{\tilde{X}}\right)=\sum_{i=1}^{n}\left|\mathbf{w}_{i}\right|+w_{\text {out }} .
$$

Let $X^{o}$ be the complement of the 0-dimensional torus orbits in $X$, or equivalently,

$$
X^{o}=\tilde{X} \backslash \bigcup_{D} D
$$

where the union is over all toric divisors $D$ of $\widetilde{X}$ with $D \notin\left\{\widetilde{D}_{1}, \ldots, \widetilde{D}_{n}, \widetilde{D}_{\text {out }}\right\}$. Every map

$$
\varphi:\left(\mathbb{P}^{1}, Q_{11}, \ldots, Q_{1 l_{1}}, \ldots, Q_{n 1}, \ldots, Q_{n l_{n}}, Q\right) \rightarrow X^{o}
$$

in $\mathfrak{M}\left(X^{o}, \mathbf{w}\right)$ yields a divisor $\varphi\left(\mathbb{P}^{1}\right) \cap D_{i}$ on each $D_{i}$. 
Lemma 4.1. The $n$ divisors

$$
\varphi\left(\mathbb{P}^{1}\right) \cap D_{1} \subseteq D_{1}, \ldots, \varphi\left(\mathbb{P}^{1}\right) \cap D_{n} \subseteq D_{n}
$$

determine $\varphi(Q) \in D_{\text {out }}$ up to $w_{\text {out }}$ choices.

Proof. The full toric boundary

$$
\iota: B \rightarrow \widetilde{X}
$$

is a simple loop of $\mathbb{P}^{1}$ 's each meeting two others. Hence, $B$ has arithmetic genus 1 and $\operatorname{Pic}_{0}(B) \cong \mathbb{C}^{*}$. We have

$$
\sum_{i=1}^{n} \varphi\left(\mathbb{P}^{1}\right) \cap D_{i}+w_{\text {out }} \cdot \varphi(Q)=\iota^{*}\left(\beta_{\mathbf{w}}\right) \in \operatorname{Pic}(B),
$$

where $\beta_{\mathbf{w}}$ here is considered an element of $\operatorname{Pic}(\widetilde{X})$. We conclude $\varphi(Q)$ is a $w_{\text {out }}$-root of

$$
\iota^{*}\left(\beta_{\mathbf{w}}\right)-\sum_{i=1}^{n} \varphi\left(\mathbb{P}^{1}\right) \cap D_{i}
$$

in $\operatorname{Pic}(B)$. There are exactly $w_{\text {out }}$ possibilities for $\varphi(Q)$ in $D_{\text {out }}$.

4.3. Stable relative maps. We first describe a partial compactification by stable relative maps,

$$
\mathfrak{M}\left(X^{o}, \mathbf{w}\right) \subseteq \overline{\mathfrak{M}}\left(X^{o}, \mathbf{w}\right) .
$$

equipped with an evaluation morphism

$$
\mathrm{ev}: \overline{\mathfrak{M}}\left(X^{o}, \mathbf{w}\right) \rightarrow \prod_{i=1}^{n}\left(D_{i}^{o}\right)^{\text {length }\left(\mathbf{w}_{i}\right)} .
$$

Consider the geometry of $\widetilde{X}$ relative to the union of the divisors

$$
\widetilde{D}_{1}, \ldots, \widetilde{D}_{n}, \widetilde{D}_{\text {out }} \subseteq \widetilde{X} \text {. }
$$

Since $\widetilde{X}$ is nonsingular and the nonsingular divisors (4.2) are disjoint, the moduli space of stable relative maps to the geometry is a well-defined Deligne-Mumford stack [11, 15, 17.

Let $\overline{\mathfrak{M}}(\widetilde{X}, \mathbf{w})$ denote the moduli space of stable relative maps of genus 0 curves representing the class $\beta_{\mathbf{w}}$ with tangency condition 8 given by

- the weight vector $\mathbf{w}_{\mathbf{i}}$ along $\widetilde{D}_{i}$,

- full tangency of order $w_{\text {out }}$ at a single point along $\widetilde{D}_{\text {out }}$.

\footnotetext{
${ }^{8}$ The relative conditions specified here are simply the orders of the tangencies at the marked points. The order of the tangency is the local intersection number. Order 1 is transverse intersection, order 2 is usual tangency, and so on. The locations of the tangencies on the relative divisors are not specified yet.
} 
The moduli space $\overline{\mathfrak{M}}(\widetilde{X}, \mathbf{w})$ parameterizes maps $\varphi$ from connected curves $C$ of arithmetic genus 0 with at worst nodal singularities to destabilizations $9 \pi$ of $\tilde{X}$ along the relative divisors,

$$
\left(C, Q_{11}, \ldots, Q_{1 l_{1}}, \ldots, Q_{n 1}, \ldots, Q_{n l_{n}}, Q\right) \stackrel{\varphi}{\rightarrow} \widetilde{\mathfrak{X}} \stackrel{\pi}{\rightarrow} \widetilde{X} .
$$

The relative conditions require $\varphi$ to be tangent to $\widetilde{D}_{\text {out }}$ at $Q$ with order $w_{\text {out }}$ and tangent to $\widetilde{D}_{i}$ at $Q_{i j}$ with order $w_{i j}$.

Let $\overline{\mathfrak{M}}\left(X^{o}, \mathbf{w}\right)$ be the open Deligne-Mumford substack of genus 0 stable relative maps to $X^{o} \subseteq \tilde{X}$. More precisely, $\overline{\mathfrak{M}}\left(X^{o}, \mathbf{w}\right)$ consists of maps (4.3) for which the composition $\pi \circ \varphi$ has image contained in $X^{o}$. Evaluation maps

$$
\mathrm{ev}: \overline{\mathfrak{M}}\left(X^{o}, \mathbf{w}\right) \rightarrow \prod_{i=1}^{n}\left(D_{i}^{o}\right)^{l_{i}}
$$

are naturally obtained. The moduli space $\mathfrak{M}\left(X^{o}, \mathbf{w}\right)$ defined in Section 3.1 is an open substack of $\overline{\mathfrak{M}}\left(X^{o}, \mathbf{w}\right)$.

While $\overline{\mathfrak{M}}\left(X^{o}, \mathbf{w}\right)$ is typically not a proper stack, the evaluation maps (4.4) are proper morphisms.

Proposition 4.2. The evaluation map ev is proper.

Proof. We use the valuative criterion for properness, so let $R$ be a valuation ring with residue field $K$, and suppose we are given the left-hand square in the following diagram:

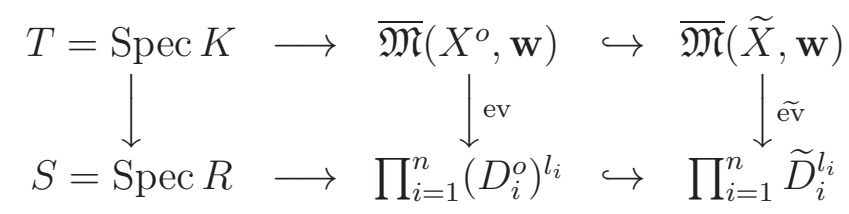

where $\widetilde{\text { ev }}$ is the corresponding evaluation map. Since $\overline{\mathfrak{M}}(\widetilde{X}, \mathbf{w})$ is proper, ev is certainly proper. Thus, we obtain a unique family of stable relative maps

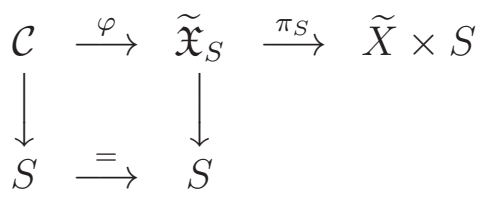

where $\widetilde{\mathfrak{X}}_{S}$ is a destabilization of $\widetilde{X} \times S$ relative to the divisor $\sum_{i=1}^{n} \widetilde{D}_{i}+\widetilde{D}_{\text {out }}$. We will show $\varphi$ is in fact a family of stable relative maps to $X^{o}$.

\footnotetext{
${ }^{9} \mathrm{~A}$ destabilization along a relative divisor is obtained by attaching a finite number of bubbles each of which is a $\mathbb{P}^{1}$-bundle over the divisor. We refer the reader to Section 1 of $[16$ for an introduction to the destabilizations required for stable relative maps. Li uses the term expanded degeneration for our destabilizations.
} 
Let $0 \in S$ be the closed point, and consider the morphism

$$
\pi_{0} \circ \varphi_{0}: \mathcal{C}_{0} \rightarrow \widetilde{X}
$$

The marked points of $\mathcal{C}_{0}$ all map to $X^{o}$. Suppose $\pi_{0} \varphi_{0}\left(\mathcal{C}_{0}\right)$ intersects a toric divisor $D \subseteq \widetilde{X}$ at a point of $D \backslash X^{o}$. Since the intersection number of $\mathcal{C}_{0}$ with $D$ is accounted for in $X^{o}$, there must be an irreducible component of $\mathcal{C}_{0}$ dominating $D$.

If $\pi_{0} \circ \varphi_{0}$ does not factor through $X^{o} \hookrightarrow \widetilde{X}$, we have shown that there must be an irreducible component $\widehat{C}$ of $\mathcal{C}_{0}$ dominating a toric divisor $D$ of $\widetilde{X}$. Let

$$
\mathcal{C} \stackrel{\xi}{\longrightarrow} \mathcal{C}^{\prime} \stackrel{\psi}{\longrightarrow} \widetilde{X} \times S
$$

be the Stein factorization of $\pi_{S} \circ \varphi$, so that $\xi$ has connected fibers and $\psi$ is finite. Let $C^{\prime}=\xi(\widehat{C})$. Since $\widehat{C}$ dominated $D, \xi$ cannot contract $\widehat{C}$. Hence $C^{\prime}$ is an irreducible component of $\mathcal{C}_{0}^{\prime}$.

Let $E_{1}$ and $E_{2}$ be the two distinct toric divisors of $\widetilde{X}$ intersecting $D$ only at two distinct toric fixed points of $D$. We will now show that there are irreducible components

$$
C_{1}^{\prime}, C_{2}^{\prime} \subseteq \mathcal{C}_{0}^{\prime}
$$

intersecting $C^{\prime}$ and dominating $E_{1}$ and $E_{2}$ respectively.

For $i \in\{1,2\}$, let $\mathcal{L}_{i}$ be the line bundle on $\widetilde{X} \times S$ corresponding to the divisor $E_{i} \times S$. Let $s_{i} \in \Gamma\left(\mathcal{L}_{i}\right)$ be a global section vanishing on $E_{i} \times S$. If $\psi^{*}\left(s_{i}\right)$ vanishes on an irreducible component $\mathcal{C}_{i}^{\prime}$ of $\mathcal{C}^{\prime}$, then the generic fiber $\left(\mathcal{C}_{i}^{\prime}\right)_{T}$ of $\mathcal{C}_{i}^{\prime} \rightarrow S$ dominates $E_{i} \times T$, contradicting the assumption that the generic fiber $\mathcal{C}_{T}$ of $\mathcal{C} \rightarrow S$ maps to $X^{o}$. Hence, $\psi^{*}\left(s_{i}\right)$ must define an effective Cartier divisor $E_{i}^{\prime}$ on $\mathcal{C}^{\prime}$. The support of $\psi^{*}\left(s_{i}\right)$ is codimension 1 in $\mathcal{C}^{\prime}$ and intersects $C^{\prime}$.

By assumption, all points of $\psi^{-1}\left(E_{i} \times T\right)$ must be the images under $\xi$ of a marked point of $\mathcal{C}_{T}$. Hence, the closure of $\psi^{-1}\left(E_{i} \times T\right)$ in $\mathcal{C}^{\prime}$ consists of images of marked points. Since the evaluation map $S \rightarrow \prod D_{i}^{l_{i}}$ factors through $\prod\left(D_{i}^{o}\right)^{l_{i}}$ by assumption 10 the closure of $\psi^{-1}\left(E_{i} \times T\right)$ in $\mathcal{C}^{\prime}$ cannot intersect $C^{\prime}$. Thus $E_{i}^{\prime}$ must contain an irreducible component $C_{i}^{\prime}$ of $\mathcal{C}_{0}^{\prime}$ intersecting $C^{\prime}$. Then, $\psi\left(C_{i}^{\prime}\right) \subseteq E_{i}$. Since $\psi$ is finite, $C_{i}^{\prime}$ must dominate $E_{i}$.

By applying the above argument repeatedly, replacing $C^{\prime}$ with $C_{1}^{\prime}$ or $C_{2}^{\prime}$, we find that either $\mathcal{C}_{0}^{\prime}$ has an infinite number of components or $\mathcal{C}_{0}^{\prime}$ contains a cycle of components dominating the union of toric divisors of $\widetilde{X}$. The first possibility is absurd. In the second case, the genus of $\mathcal{C}_{0}^{\prime}$ would have to be at least 1 . Since $\left.\xi\right|_{\mathcal{C}_{0}}: \mathcal{C}_{0} \rightarrow \mathcal{C}_{0}^{\prime}$

\footnotetext{
${ }^{10}$ The assumption covers all the marked points except $Q$. However, the image of $Q$ is constrained by the images of the other marked points by Lemma 4.1 and therefore cannot intersect $C^{\prime}$ even in the closure.
} 
has connected fibers, $\mathcal{C}_{0}$ would also have genus at least 1 , contradicting the genus 0 assumption.

We have shown by contradiction that $\varphi: \mathcal{C} \rightarrow \widetilde{\mathfrak{X}}_{S}$ must in fact be a family of stable relative maps to $X^{o}$.

Maps parameterized by $\overline{\mathfrak{M}}\left(X^{o}, \mathbf{w}\right)$ have a rather simple structure. Let

$$
\left(C, Q_{11}, \ldots, Q_{1 l_{1}}, \ldots, Q_{n 1}, \ldots, Q_{n l_{n}}, Q\right) \stackrel{\varphi}{\rightarrow} \mathfrak{X}^{o} \stackrel{\pi}{\rightarrow} X^{o}
$$

be an element of $\overline{\mathfrak{M}}\left(X^{o}, \mathbf{w}\right)$. Then, the irreducible components $P \subseteq C$ are of exactly two distinct types

(i) $\left.\pi \circ \varphi\right|_{P}: P \rightarrow X^{\circ}$ is a constant map, or

(ii) $\left.\pi \circ \varphi\right|_{P}: P \rightarrow X^{o}$ is an element of $\mathfrak{M}\left(X^{o}, \mathbf{w}^{P}\right)$ for possibly different weight vectors $\mathbf{w}^{P}=\left(\mathbf{w}_{1}^{P}, \ldots, \mathbf{w}_{n}^{P}\right)$, with not all $\mathbf{w}_{i}^{P}=\emptyset$.

The possibilities are restricted to (i) and (ii) because no components of $C$ may both map to a destabilizing component and surject onto the corresponding relative divisor.

The following result shows the domain $C$ is irreducible if the locations of the relative conditions are specified generically.

Proposition 4.3. The general fiber of

$$
\text { ev }: \overline{\mathfrak{M}}\left(X^{o}, \mathbf{w}\right) \rightarrow \prod_{i=1}^{n}\left(D_{i}^{o}\right)^{\text {length }\left(\mathbf{w}_{i}\right)}
$$

is contained in $\mathfrak{M}\left(X^{o}, \mathbf{w}\right)$.

Proof. Let $C \stackrel{\varphi}{\rightarrow} \mathfrak{X}^{o} \stackrel{\pi}{\rightarrow} X^{o}$ be an element of the general fiber of the evaluation map. Consider first the component $P \subseteq C$ containing the point $Q$. Suppose $P$ is of type (i). Let

$$
B_{1}, B_{2}, \ldots, B_{r} \subseteq \mathfrak{X}^{o}
$$

be the destabilizing components over $D_{\text {out }}^{o}$ with

$$
X^{o} \cap B_{1} \neq \emptyset
$$

and

$$
B_{i} \cap B_{i+1} \neq \emptyset, \quad 1 \leq i \leq r-1 .
$$

Each $B_{i}$ is a $\mathbb{P}^{1}$ bundle over $D_{\text {out }}^{o}$ relative to two disjoint sections. Since $P$ contains $Q$, the image of $P$ lies in $B_{r}$. By the definition of a stable relative map, we conclude

(a) The intersection of the image of $C$ with $\cup_{k=1}^{r} B_{k}$ is a single chain of $\mathbb{P}^{1}$-fibers which intersects $X^{o}$ at a point $x \in D_{\text {out }}^{o}$.

(b) There are at least two type (ii) components of $C$ intersecting $D_{\text {out }}^{o}$ at $x$. 
The conclusion (b) follows from the stability assumption on the maps of components of $C$ to $\cup_{k=1}^{r} B_{k}$.

Let $P_{1}, P_{2} \subseteq C$ be two components of type (ii) intersecting $D_{\text {out }}^{o}$ at $x$. The restrictions of $\pi \circ \varphi$ to $P_{1}$ and $P_{2}$ determines elements of $\mathfrak{M}\left(X^{o}, \mathbf{w}^{P_{1}}\right)$ and $\mathfrak{M}\left(X^{o}, \mathbf{w}^{P_{2}}\right)$ respectively. By the genericity assumption, both restrictions lie in the general fibers of the evaluation maps

$$
\begin{aligned}
\text { ev }: \overline{\mathfrak{M}}\left(X^{o}, \mathbf{w}^{P_{1}}\right) & \rightarrow \prod_{i=1}^{n}\left(D_{i}^{o}\right)^{\operatorname{length}\left(\mathbf{w}_{i}^{P_{1}}\right)}, \\
\mathrm{ev}: \overline{\mathfrak{M}}\left(X^{o}, \mathbf{w}^{P_{2}}\right) & \rightarrow \prod_{i=1}^{n}\left(D_{i}^{o}\right)^{\operatorname{length}\left(\mathbf{w}_{i}^{P_{2}}\right)} .
\end{aligned}
$$

But the incidence point $x$ for the two restrictions is easily seen not to agree for elements of general fibers of the evaluation maps by Lemma 4.1 .

We have shown the component $P$ containing $Q$ must be of type (ii) and no destabilization occurs over $D_{\text {out }}^{o}$. Any other component $P^{\prime} \subseteq C$ of type (ii) cannot intersect $D_{\text {out }}^{o}$ since all intersection have been accounted for. But by Proposition 3.3 and the genericity statement, no such curves $P^{\prime}$ exist. Hence, $P$ is the unique type (ii) component.

There are now only two possibilities for type (i) components $\widehat{P} \subseteq C$. First, $\widehat{P}$ could be contracted to a point of $X^{o}$ away from the toric divisors. However, then $\widehat{P}$ would have to intersect $P$ in an least three points, violating the genus condition on $C$. Second, $\widehat{P}$ could be contracted to a point of $D_{i}^{o}$. Then, let

$$
B_{1}^{i}, B_{2}^{i}, \ldots, B_{s}^{i} \subseteq \mathfrak{X}^{o}
$$

be the destabilizing components over $D_{i}^{o}$. The component $\widehat{P}$ distinguishes a chain of $\mathbb{P}^{1}$-fibers of $\cup_{k=1}^{s} B_{k}^{i}$ which intersect $X^{o}$ at a point $y \in D_{i}^{o}$. Let $\widehat{P}_{0} \subseteq C$ be a component which maps to the last element of the chain in $B_{s}^{i}$. By the genericity of the fiber of the evaluation map, $\widehat{P}_{0}$ is unique. The components of $C$ mapping to the chain connect $\widehat{P}_{0}$ with $P$. These components must themselves be a chain by the genus 0 condition on $C$.

The last argument applies to every component $\widehat{P}$ of type (i) mapping to $D_{i}^{o}$. Hence, we conclude the stability of relative map is violated over $D_{i}^{o}$. Therefore, $C$ has a single type (ii) component and no type (i) components.

4.4. Gromov-Witten theory for $X^{o}$. The moduli space $\overline{\mathfrak{M}}\left(X^{o}, \mathbf{w}\right)$ carries a perfect obstruction theory and a virtual class

$$
\left[\overline{\mathfrak{M}}\left(X^{o}, \mathbf{w}\right)\right]^{\text {vir }} \in A_{*}\left(\overline{\mathfrak{M}}\left(X^{o}, \mathbf{w}\right), \mathbb{Q}\right)
$$


of dimension

$$
\int_{\beta(\mathbf{w})} c_{1}\left(T_{X^{o}}\right)-1-\sum_{i=1}^{n} \sum_{j=1}^{l_{i}}\left(w_{i j}-1\right)-\left(w_{\text {out }}-1\right)=\sum_{i=1}^{n} \operatorname{length}\left(\mathbf{w}_{i}\right),
$$

see [11, 15, 17].

Since $\overline{\mathfrak{M}}\left(X^{o}, \mathbf{w}\right)$ is not a proper Deligne-Mumford stack, obtaining numerical invariants from the virtual class is not always possible. However, the properness of the evaluation map

$$
\mathrm{ev}: \overline{\mathfrak{M}}\left(X^{o}, \mathbf{w}\right) \rightarrow \prod_{i=1}^{n}\left(D_{i}^{o}\right)^{l_{i}}
$$

proven in Proposition 4.2 may be used to define the invariants we will need.

Select a point of $\prod_{i=1}^{n}\left(D_{i}^{o}\right)^{l_{i}}$, and let

$$
\gamma: \operatorname{Spec}(\mathbb{C}) \rightarrow \prod_{i=1}^{n}\left(D_{i}^{o}\right)^{l_{i}}
$$

be the associated inclusion map. The properness of (4.6) implies

$$
F(\gamma)=\operatorname{Spec}(\mathbb{C}) \times_{\prod_{i=1}^{n}\left(D_{i}^{o}\right)^{l_{i}}} \overline{\mathfrak{M}}\left(X^{o}, \mathbf{w}\right)
$$

is a proper Deligne-Mumford stack. Define the relative Gromov-Witten invariant

$$
N_{\mathbf{m}}^{\mathrm{rel}}(\mathbf{w})=\int_{F(\gamma)} \gamma^{!}\left(\left[\overline{\mathfrak{M}}\left(X^{o}, \mathbf{w}\right)\right]^{\text {vir }}\right)
$$

where integration is given as usual by proper push-forward to a point.

Since the codimension of the inclusion $\gamma$ equals the virtual dimension of $\overline{\mathfrak{M}}\left(X^{o}, \mathbf{w}\right)$, definition (4.7) is sensible. The properness of the evaluation map (4.6) implies $N_{\mathbf{m}}^{\mathrm{rel}}(\mathbf{w})$ is independent of the point $\gamma$.

The invariant $N_{\mathbf{m}}^{\mathrm{rel}}(\mathbf{w})$ is the virtual count of genus 0 maps to $X^{o}$ of class $\beta_{\mathbf{w}}$ with tangency conditions $\mathbf{w}_{i}$ at specified points of $D_{i}^{o}$ and a tangency condition $w_{\text {out }}$ at an unspecified point of $D_{\text {out }}^{o}$. The actual moduli spaces of such maps may vary with different choices of the locations of the tangency conditions. For example, dimensions may jump. But the virtual count $N_{\mathbf{m}}^{\mathrm{rel}}(\mathbf{w})$ does not change.

Theorem 4.4. $N_{\mathbf{m}}^{\mathrm{hol}}(\mathrm{w})=N_{\mathbf{m}}^{\mathrm{rel}}(\mathrm{w})$.

Proof. We can compute $N_{\mathbf{m}}^{\mathrm{rel}}(\mathbf{w})$ for a general point

$$
\gamma: \operatorname{Spec}(\mathbb{C}) \rightarrow \prod_{i=1}^{n}\left(D_{i}^{o}\right)^{l_{i}}
$$

By Proposition 4.3, the fiber product $F(\gamma)$ embeds in $\mathfrak{M}\left(X^{o}, \mathbf{w}\right)$. By Proposition 3.2. $\mathfrak{M}\left(X^{o}, \mathbf{w}\right)$ is a nonsingular Deligne-Mumford stack of the expected dimension. 
Hence, the virtual class of $\mathfrak{M}\left(X^{o}, \mathbf{w}\right)$ is the usual fundamental class. By Bertini, the fiber product $F(\gamma)$ is a finite set of reduced points. By definition, $N_{\mathbf{m}}^{\mathrm{rel}}(\mathbf{w})$ equals the cardinality of $F(\gamma)$. On the other hand, the cardinality of $F(\gamma)$ is simply the degree of

$$
\text { ev }: \mathfrak{M}\left(X^{o}, \mathbf{w}\right) \rightarrow \prod_{i=1}^{n}\left(D_{i}^{o}\right)^{l_{i}}
$$

which is $N_{\mathbf{m}}^{\mathrm{hol}}(\mathbf{w})$.

\section{Formulas in the tropical Vertex Group}

5.1. Simple blow-ups. Let $\mathbf{P}=\left(\mathbf{P}_{1}, \ldots, \mathbf{P}_{n}\right)$ be an $n$-tuple of ordered partitions where

$$
\mathbf{P}_{i}=p_{i 1}+\ldots+p_{i \ell_{i}}
$$

We continue to treat the nondegenerate case where the ray generated by

$$
0 \neq m_{\text {out }}=\sum_{i=1}^{n}\left|\mathbf{P}_{i}\right| m_{i}, \quad m_{\text {out }}=p_{\text {out }} m_{\text {out }}^{\prime}
$$

is distinct from the rays generated by $-m_{i}$. Following the notation of Section 4.2, let $X^{o}$ be the corresponding open toric surface with divisors $D_{1}^{o}, \ldots, D_{n}^{o}, D_{\text {out }}^{o}$.

Select distinct points $x_{i 1}, \ldots, x_{i \ell_{i}} \in D_{i}^{o}$ corresponding to the parts of $\mathbf{P}_{i}$. Let

$$
\nu: \tilde{X}[\mathbf{P}] \rightarrow \widetilde{X}
$$

be the blow-up of $\widetilde{X}$ along all the points $x_{i j}$, and let

$$
X^{o}[\mathbf{P}]=\nu^{-1}\left(X^{o}\right)
$$

Let $E_{i j} \subseteq \tilde{X}[\mathbf{P}]$ be the exceptional divisor over $x_{i j}$. Let $\beta \in H_{2}(\tilde{X}, \mathbb{Z})$ be determined by intersection numbers with toric divisors,

$$
\widetilde{D}_{i} \cdot \beta=\left|\mathbf{P}_{i}\right|, \quad \widetilde{D}_{\text {out }} \cdot \beta=p_{\text {out }}
$$

and $D \cdot \beta=0$ if $D \notin\left\{\widetilde{D}_{1}, \ldots, \widetilde{D}_{n}, \widetilde{D}_{\text {out }}\right\}$. Let

$$
\beta_{\mathbf{P}}=\nu^{*}(\beta)-\sum_{i=1}^{n} \sum_{j=1}^{\ell_{i}} p_{i j}\left[E_{i j}\right] \in H_{2}(\tilde{X}[\mathbf{P}], \mathbb{Z}) .
$$

Let $\overline{\mathfrak{M}}\left(\widetilde{X}[\mathbf{P}] / \widetilde{D}_{\text {out }}\right)$ be the moduli of stable relative maps of genus 0 curves representing the class $\beta_{\mathbf{P}}$ with full tangency of order $p_{\text {out }}$ at an unspecified point of $\widetilde{D}_{\text {out }}$. Let

$$
\overline{\mathfrak{M}}\left(X^{o}[\mathbf{P}] / D_{\text {out }}^{o}\right) \subseteq \overline{\mathfrak{M}}\left(\widetilde{X}[\mathbf{P}] / \widetilde{D}_{\text {out }}\right)
$$

be the open subspace of maps which avoid $\tilde{X}[\mathbf{P}] \backslash X^{o}[\mathbf{P}]$. Unlike the theories considered in Section 4, the geometry $X^{o}[\mathbf{P}] / D_{\text {out }}^{o}$ is relative to a single irreducible divisor. 
5.2. Gromov-Witten theory. The virtual dimension of $\overline{\mathfrak{M}}\left(X^{o}[\mathbf{P}] / D_{\text {out }}^{o}\right)$ is calculated by unwinding the definitions,

$$
\begin{aligned}
\operatorname{virdim}_{\mathbb{C}} \overline{\mathfrak{M}}\left(X^{o}[\mathbf{P}] / D_{\text {out }}^{o}\right) & =\int_{\beta_{\mathbf{P}}} c_{1}\left(T_{X^{o}[P]}\right)-1-\left(p_{\text {out }}-1\right) \\
& =\sum_{i=1}^{n}\left|\mathbf{P}_{i}\right|-\sum_{i=1}^{n} \sum_{j=1}^{\ell_{i}} p_{i j}+p_{\text {out }}-1-\left(p_{\text {out }}-1\right) \\
& =0 .
\end{aligned}
$$

Proposition 5.1. $\overline{\mathfrak{M}}\left(X^{o}[\mathbf{P}] / D_{\text {out }}^{o}\right)$ is proper over $\operatorname{Spec}(\mathbb{C})$.

Proof. The argument exactly follows the proof of Proposition 4.2. Let

$$
(C, Q) \stackrel{\varphi}{\rightarrow} \mathfrak{X}^{o}[\mathbf{P}] \stackrel{\pi}{\rightarrow} X^{o}[\mathbf{P}]
$$

be an element of $\overline{\mathfrak{M}}\left(X^{o}[\mathbf{P}] / D_{\text {out }}^{o}\right)$. Here, $\mathfrak{X}^{o}[\mathbf{P}]$ is a destabilization of $X^{o}[\mathbf{P}]$ along the relative divisor $D_{\text {out }}^{o}$. If we consider the composition

$$
\nu \circ \pi \circ \varphi: C \rightarrow X^{\circ} \subseteq \widetilde{X}
$$

then the intersections with all the toric divisors of $\widetilde{X}$ are specified - occurring at the points $x_{i j}$ in $D_{i}^{o}$ and the image of $Q$ in $D_{\text {out }}^{o}$. The limits in $\overline{\mathfrak{M}}\left(\tilde{X}[\mathbf{P}] / \widetilde{D}_{\text {out }}\right)$ of 1-parameter families of elements of $\overline{\mathfrak{M}}\left(X^{o}[\mathbf{P}] / D_{\text {out }}^{o}\right)$ cannot meet points of

$$
\tilde{X}[\mathbf{P}] \backslash X^{o}[\mathbf{P}] \text {. }
$$

Otherwise, an entire strict transform of a toric divisor of $\widetilde{X}$ would lie in the image of the limit, and the loop construction of the proof of Proposition 4.2 can be made.

We define the Gromov-Witten invariant $N_{\mathbf{m}}[\mathbf{P}]$ by the usual integral,

$$
N_{\mathbf{m}}[\mathbf{P}]=\int_{\left[\overline{\mathfrak{M}}\left(X^{o}[\mathbf{P}] / D_{\text {out }}^{o}\right)\right]^{v i r}} 1 .
$$

The properness Proposition 5.1 holds relatively as the centers $x_{i j} \in D_{i}^{o}$ of the blow-ups of $\widetilde{X}$ are moved along $D_{i}^{o}$. Hence, the integral $N_{\mathbf{m}}[\mathbf{P}]$ is independent of the locations of the distinct points $x_{i j} \in D_{i}^{o}$.

5.3. Degeneration. Let $\mathbb{C}$ be the affine line. Let

$$
X^{o} \times \mathbb{C} \rightarrow \mathbb{C}
$$

be the trivial family over $\mathbb{C}$. The standard degeneration of $X^{o}$ relative to $D_{1}^{o}, \ldots, D_{n}^{o}, D_{\text {out }}^{o}$ over $\mathbb{C}$,

$$
\epsilon: \mathcal{F} \rightarrow \mathbb{C}
$$


is obtained by blowing-up the loci $D_{1}^{o}, \ldots D_{n}^{o}, D_{\text {out }}^{o}$ over $0 \in \mathbb{C}$ in (5.1). After blowing-up the sections of $\epsilon$ corresponding to the points $x_{i j}$, we obtain

$$
\epsilon_{\mathbf{P}}: \mathcal{F}[\mathbf{P}] \rightarrow \mathbb{C}
$$

For $\xi \neq 0$, we have $\epsilon_{\mathbf{P}}^{-1}(\xi) \cong X^{o}[\mathbf{P}]$. The special fiber has a different form

$$
\epsilon_{\mathbf{P}}^{-1}(0)=X^{o} \cup \bigcup_{i=1}^{n} B^{o}\left[\mathbf{P}_{i}\right]
$$

where $B^{o}\left[\mathbf{P}_{i}\right]$ is the blow-up of $\mathbb{P}\left(\mathcal{O}_{D_{i}^{o}} \oplus \operatorname{Norm}_{X^{\circ} / D_{i}^{o}}\right)$ at the limits

$$
\bar{x}_{i 1}, \ldots, \bar{x}_{i \ell_{i}}
$$

of the points $x_{i j}$.

The moduli spaces of stable relative maps to the fibers of $\epsilon_{\mathbf{P}}$ over $\xi \neq 0$ are canonically isomorphic to $\overline{\mathfrak{M}}\left(X^{o}[\mathbf{P}] / D_{\text {out }}^{o}\right)$. The limits of stable relative maps to $\epsilon_{\mathbf{P}}^{-1}(\xi)$ as $\xi \rightarrow 0$ are well-understood in relative Gromov-Witten theory [11, 15, 17]. The limit is a union of stable maps to the relative geometries determined by the components of the special fiber over 0. A priori, the limit may leave the open subspaces we are considering. However, the properness argument (used twice already) easily shows the limit is a union of stable maps to the following $n+1$ open relative geometries

$$
X^{o} / D_{1}^{o} \cup \ldots D_{n}^{o} \cup D_{\text {out }}^{o}, \quad B^{o}\left[\mathbf{P}_{1}\right] / D_{1}^{o}, \ldots, \quad B^{o}\left[\mathbf{P}_{n}\right] / D_{n}^{o},
$$

with matching conditions along the common relative divisors.

Relative maps to $B^{o}\left[\mathbf{P}_{i}\right] / D_{i}^{o}$ are simple to describe. Let $\bar{E}_{i j} \subseteq B^{o}\left[\mathbf{P}_{i}\right]$ be the exceptional divisor of

$$
B^{o}\left[\mathbf{P}_{i}\right] \rightarrow \mathbb{P}\left(\mathcal{O}_{D_{i}^{o}} \oplus \operatorname{Norm}_{X^{o} / D_{i}^{o}}\right)
$$

over $\bar{x}_{i j}$. Let $C_{i j} \subseteq B^{o}\left[\mathbf{P}_{i}\right]$ be the strict transform to $B^{o}\left[\mathbf{P}_{i}\right]$ of the unique fiber of

$$
\mathbb{P}\left(\mathcal{O}_{D_{i}^{o}} \oplus \operatorname{Norm}_{X^{o} / D_{i}^{o}}\right) \rightarrow D_{i}^{o}
$$

containing $\bar{x}_{i j}$. A relative map to $B^{o}\left[\mathbf{P}_{i}\right] / D_{i}^{o}$ with a connected domain and class $d\left[C_{i j}\right]$ must be a $d$-fold multiple cover of $C_{i j}$.

We can calculate all the invariants of $B^{o}\left[\mathbf{P}_{i}\right]$ relative to $D_{i}^{o}$ which we will require. Let

$$
\overline{\mathfrak{M}}\left(B^{o}\left[\mathbf{P}_{i}\right] / D_{i}^{o}, d\right)_{j}
$$

be the moduli space of genus 0 relative maps of class $d\left[C_{i j}\right]$ with full tangency of order $d$ along $D_{i}^{0}$. Let $\overline{\mathfrak{M}}\left(C_{i j} / \infty, d\right)$ be the moduli space of genus 0 relative maps of degree $d$ with ramification of order $d$ over $\infty=C_{i j} \cap D_{i}^{o}$. As spaces,

$$
\overline{\mathfrak{M}}\left(B^{o}\left[\mathbf{P}_{i}\right] / D_{i}^{o}, d\right)_{j}=\overline{\mathfrak{M}}\left(C_{i j} / \infty, d\right) .
$$


However, the two moduli spaces (5.4) carry obstruction theories which differ by

$$
H^{1}\left(C, \varphi^{*}\left(\operatorname{Norm}_{B^{o}\left[\mathbf{P}_{i}\right] / C_{i j}}\right)\right),
$$

at the moduli point $\left[\varphi: C \rightarrow C_{i j}\right]$. The virtual dimension of $\overline{\mathfrak{M}}\left(B^{o}\left[\mathbf{P}_{i}\right] / D_{i}^{o}, d\right)_{j}$ is easily seen to be 0 . Let

$$
R_{d}=\int_{\left.\overline{[\overline{\mathfrak{M}}}\left(B^{o}\left[\mathbf{P}_{i}\right] / D_{i}^{o}, d\right)_{j}\right]^{v i r}} 1 .
$$

Proposition 5.2. $R_{d}=\frac{(-1)^{d-1}}{d^{2}}$.

Proof. The integral $R_{d}$ is

$$
\begin{aligned}
R_{d} & =\int_{\left.\overline{[\bar{M}}\left(C_{i j} / \infty, d\right)\right]^{v i r}} e\left(H^{1}\left(C, \varphi^{*}\left(\operatorname{Norm}_{B^{o}\left[\mathbf{P}_{i}\right] / C_{i j}}\right)\right)\right) \\
& =\int_{\left[\overline{\mathfrak{M}}\left(\mathbb{P}^{1} / \infty, d\right)\right]^{v i r}} e\left(H^{1}\left(C, \varphi^{*}\left(\mathcal{O}_{\mathbb{P}^{1}}(-1)\right)\right)\right) \\
& =\frac{(-1)^{d-1}}{d^{2}} .
\end{aligned}
$$

In the first and second lines, $e(V)$ denotes the Euler class (top Chern class) of the vector bundle on the moduli space of stable relative maps with fibers defined by $V$. The evaluation in the last equality is the genus 0 part 11 of Theorem 5.1 of [2].

An $n$-tuple $\mathbf{w}$ of weight vectors is of the same type as an $n$-tuple $\mathbf{P}$ of ordered partitions if $\left|\mathbf{w}_{i}\right|=\left|\mathbf{P}_{i}\right|$ for all $i$. A set partition of $\mathbf{w}_{i}$ compatible with $\mathbf{P}_{i}$ is a disjoint union 12

$$
I_{1} \cup \ldots \cup I_{\ell_{i}}=\left\{1, \ldots, l_{i}\right\}
$$

satisfying

$$
p_{i j}=\sum_{r \in I_{j}} w_{i r}
$$

for all $j$. Let

$$
R_{\mathbf{P}_{i} \mid \mathbf{w}_{i}}=\sum_{I_{\bullet}} \prod_{j=1}^{l_{i}} R_{w_{i j}}
$$

where the sum is over all set partitions $I_{\bullet}$ of $\mathbf{w}_{i}$ compatible with $\mathbf{P}_{i}$.

\footnotetext{
11 The local Gromov-Witten theory of curves is proven in 3] to determine a TQFT. As such, the entire theory is specified by values on the cap, the tube, and the pair of pants. The integral $R_{d}$ arises in the genus 0 part of the cap contribution. In fact, the full genus $g$ cap is computed in [2] by Hodge integral techniques.

${ }^{12}$ Recall the length of $\mathbf{w}_{i}$ is $l_{i}$ and the length of $\mathbf{P}_{i}$ is $\ell_{i}$. The set $I_{j}$ is allowed to be empty if $p_{i j}=0$.
} 
The degeneration formula for relative Gromov-Witten theory applied to our setting yields the following result.

Proposition 5.3. We have

$$
N_{\mathbf{m}}[\mathbf{P}]=\sum_{\mathbf{w}} N_{\mathbf{m}}^{\mathrm{rel}}(\mathbf{w}) \cdot \prod_{i=1}^{n} \frac{\prod_{j=1}^{l_{i}} w_{i j}}{\left|\operatorname{Aut}\left(\mathbf{w}_{i}\right)\right|} \cdot R_{\mathbf{P}_{i} \mid \mathbf{w}_{i}}
$$

where the sum is over all $n$-tuples of weight vectors $\mathbf{w}$ of the same type as $\mathbf{P}$.

Proof. We simply apply the degeneration formula of relative Gromov-Witten theory [11, 15, 17] to the family $\epsilon_{\mathbf{P}}$ of (5.3). Our properness results show the formula both makes sense and is valid in the open geometry at hand. The left term $N_{\mathbf{m}}^{\mathrm{rel}}(\mathbf{w})$ is the contribution of $X^{o}$. The numerator

$$
\prod_{i=1}^{n} \prod_{j=1}^{l_{i}} w_{i j}
$$

is the standard multiplicity in the degeneration formula. The ratio

$$
\frac{R_{\mathbf{P}_{i} \mid \mathbf{w}_{i}}}{\left|\operatorname{Aut}\left(\mathbf{w}_{i}\right)\right|}
$$

is exactly the correct automorphism weighted contribution of the component $B^{o}\left[\mathbf{P}_{i}\right]$.

5.4. First commutator formulas. We can now prove our first formulas in the tropical vertex group. Let $\mathfrak{d}_{1} \neq \mathfrak{d}_{2}$ be two lines through the origin in $M_{\mathbb{R}}$. Let $\mathbf{m}=\left(m_{1}, m_{2}\right)$ be the two associated primitive vectors. Let

$$
f_{\mathfrak{d}_{1}}=\prod_{\xi=1}^{\ell_{1}}\left(1+s_{\xi} z^{m_{1}}\right), \quad f_{\mathfrak{d}_{2}}=\prod_{\xi=1}^{\ell_{2}}\left(1+t_{\xi} z^{m_{2}}\right)
$$

be functions over the complete ring $\mathbb{C}\left[\left[s_{1}, \ldots, s_{\ell_{1}}, t_{1}, \ldots, t_{\ell_{2}}\right]\right]$. Consider the scattering diagram

$$
\mathfrak{D}=\left\{\left(\mathfrak{d}_{1}, f_{\mathfrak{d}_{1}}\right),\left(\mathfrak{d}_{2}, f_{\mathfrak{d}_{2}}\right)\right\}
$$

Let $S(\mathfrak{D})$ be the unique minimal scattering diagram (obtained by adding rays to $\mathfrak{D}$ ) for which the path ordered product around the origin is trivial. Let $m_{\text {out }}^{\prime} \in M$ be a primitive vector. What is the associated function $f_{m_{\text {out }}^{\prime}}$ ?

We may write the scattering diagram (5.6) equivalently with $\ell_{1}+\ell_{2}$ lines,

$$
\mathfrak{D}=\left\{\left(\mathfrak{d}_{1}, 1+s_{1} z^{m_{1}}\right), \ldots,\left(\mathfrak{d}_{1}, 1+s_{\ell_{1}} z^{m_{1}}\right),\left(\mathfrak{d}_{2}, 1+t_{1} z^{m_{2}}\right), \ldots,\left(\mathfrak{d}_{2}, 1+t_{\ell_{2}} z^{m_{2}}\right)\right\} .
$$

Then, $\mathfrak{D}$ is standard by Definition 1.10 , and Theorem 2.8 determines $f_{m_{\text {out }}^{\prime}}$ in terms of tropical geometry. There are three possibilities 
(i) $m_{\text {out }}^{\prime} \notin \mathbb{Q} \geq 0 m_{1}+\mathbb{Q}_{\geq 0} m_{2}$,

(ii) $m_{\text {out }}^{\prime}=m_{1}$ or $m_{\text {out }}^{\prime}=m_{2}$,

(iii) $m_{\text {out }}^{\prime} \in \mathbb{Q}_{>0} m_{1}+\mathbb{Q}_{>0} m_{2}$,

The outcomes in cases (i) and (ii) are straightforward. By Theorem $2.8, f_{m_{\text {out }}^{\prime}}=1$ in case (i) and $f_{m_{\text {out }}^{\prime}}$ equals either $f_{\mathfrak{d}_{1}}$ or $f_{\mathfrak{d}_{2}}$ respectively. The interesting case is (iii).

For ordered partitions $\mathbf{P}_{1}=p_{11}+\ldots+p_{1 \ell_{1}}$ and $\mathbf{P}_{2}=p_{21}+\ldots+p_{2 \ell_{2}}$, let

$$
s^{\mathbf{P}_{1}}=\prod_{\xi=1}^{\ell_{1}} s_{\xi}^{p_{1 \xi}}, \quad t^{\mathbf{P}_{2}}=\prod_{\xi=1}^{\ell_{2}} t_{\xi}^{p_{2 \xi}}
$$

be the corresponding monomials.

Theorem 5.4. If $m_{\text {out }}^{\prime} \in \mathbb{Q}_{>0} m_{1}+\mathbb{Q}_{>0} m_{2}$, then

$$
\log f_{m_{\text {out }}^{\prime}}=\sum_{k=1}^{\infty} \sum_{\mathbf{P}=\left(\mathbf{P}_{\mathbf{1}}, \mathbf{P}_{\mathbf{2}}\right)} k N_{\mathbf{m}}[\mathbf{P}] s^{\mathbf{P}_{1}} t^{\mathbf{P}_{2}} z^{k m_{\text {out }}^{\prime}}
$$

where the sum is over all ordered partitions $\mathbf{P}_{i}$ of length $\ell_{i}$ satisfying

$$
\left|\mathbf{P}_{1}\right| m_{1}+\left|\mathbf{P}_{2}\right| m_{2}=k m_{\text {out }}^{\prime} .
$$

Proof. To apply Theorem [2.8, we first calculate the Taylor series

$$
\begin{aligned}
& \log \left(1+s_{\xi} z^{m_{1}}\right)=\sum_{d=1}^{\infty} d \frac{(-1)^{d-1}}{d^{2}} z^{d m_{1}} s_{\xi}^{d}, \\
& \log \left(1+t_{\xi} z^{m_{2}}\right)=\sum_{d=1}^{\infty} d \frac{(-1)^{d-1}}{d^{2}} z^{d m_{2}} t_{\xi}^{d} .
\end{aligned}
$$

In both of the above cases, the $a$ coefficients of Theorem 2.8 match the relative invariants $R_{d}$ computed in Proposition 5.2 .

$$
a_{\xi d d}=R_{d}=\frac{(-1)^{d-1}}{d^{2}}
$$

By Theorems 2.8, 3.4, and 4.4,

$$
\log f_{m_{\text {out }}^{\prime}}=\sum_{k=1}^{\infty} \sum_{\mathbf{P}=\left(\mathbf{P}_{\mathbf{1}}, \mathbf{P}_{\mathbf{2}}\right)} \sum_{\mathbf{w}} k N_{\mathbf{m}}^{\mathrm{rel}}(\mathbf{w})\left(\prod_{i=1}^{2} \frac{\prod_{j=1}^{l_{i}} w_{i j}}{\left|\operatorname{Aut}\left(\mathbf{w}_{i}\right)\right|} \cdot R_{\mathbf{P}_{i} \mid \mathbf{w}_{i}}\right) s^{\mathbf{P}_{1}} t^{\mathbf{P}_{2}} z^{k m_{\text {out }}^{\prime}},
$$

where the second sum is over all ordered partitions $\mathbf{P}=\left(\mathbf{P}_{1}, \mathbf{P}_{2}\right)$ of lengths $\left(\ell_{1}, \ell_{2}\right)$ satisfying

$$
\left|\mathbf{P}_{1}\right| m_{1}+\left|\mathbf{P}_{2}\right| m_{2}=k m_{\text {out }}^{\prime}
$$

and the third sum is over all weight vectors $\mathbf{w}=\left(\mathbf{w}_{1}, \mathbf{w}_{2}\right)$ of the same type as $\mathbf{P}$. The result then follows from Proposition 5.3 . 
Theorem 5.4 computes the commutators of $\theta_{\mathfrak{d}_{1}, f_{\mathfrak{o}_{1}}}$ and $\theta_{\mathfrak{d}_{2}, f_{\mathfrak{o}_{2}}}$ via slope ordered products in the tropical vertex group in the form discussed in Section 0.4. Theorem 5.4 specializes to Theorem 0.1, but is a much better statement. By working over the ring $\mathbb{C}\left[\left[s_{1}, \ldots, s_{\ell_{1}}, t_{1}, \ldots, t_{\ell_{2}}\right]\right]$, we see computing the commutator is precisely equivalent to calculating all the Gromov-Witten invariants $N_{\mathbf{m}}\left[\left(\mathbf{P}_{1}, \mathbf{P}_{2}\right)\right]$ for ordered partitions $\mathbf{P}_{i}$ of length $\ell_{i}$.

Of course, functions $f_{1}$ and $f_{2}$ may not be in the form specified by (5.5). The general commutator formula is expressed in terms of orbifold blow-ups of toric surfaces.

5.5. Orbifold blow-ups. Let $D \subseteq S$ be a nonsingular divisor contained in a surface. Let $p \in D$. Let $p_{D}^{r}$ be the unique length $r$ subscheme of $D$ with support $p$. Let

$$
S_{r} \rightarrow S
$$

be the blow-up of $S$ along $p_{D}^{r}$. Of course, $S_{1}$ is the usual blow-up of $S$ along $p$. For $r \geq 2, S_{r}$ has a unique $A_{r-1}$-singularity. Hence, $S_{r}$ admits a unique structure as a nonsingular Deligne-Mumford stack $\mathcal{S}_{r} \rightarrow S_{r}$. We call the composition

$$
\delta_{r}: \mathcal{S}_{r} \rightarrow S
$$

the r-orbifold blow-up of $S$ along $(p, D)$

The exceptional divisor $E \subseteq \mathcal{S}_{r}$ of $\delta_{r}$ is a $\mathbb{P}^{1}$ with a single $r$-fold stack point lying above the original $A_{r-1}$-singularity. The self-intersection is

$$
[E]^{2}=-\frac{1}{r}
$$

A graded partition consists of a finite sequence $\left(\mathbf{P}^{1}, \mathbf{P}^{2}, \ldots, \mathbf{P}^{d}\right)$ of ordered partitions for which every part of $\mathbf{P}^{r}$ is divisible by $r$. The length of a graded partition is given by a $d$-tuple $\left(\ell^{1}, \ldots, \ell^{d}\right)$. The size of a graded partition is $\sum_{r=1}^{d}\left|\mathbf{P}^{r}\right|$.

Let $\mathbf{G}=\left(\mathbf{G}_{1}, \ldots, \mathbf{G}_{n}\right)$ be an $n$-tuple of graded partitions,

$$
\mathbf{G}_{i}=\left(\mathbf{P}_{i}^{1}, \mathbf{P}_{i}^{2}, \ldots, \mathbf{P}_{i}^{d_{i}}\right)
$$

We treat the nondegenerate case where the ray generated by

$$
0 \neq m_{\text {out }}=\sum_{i=1}^{n}\left|\mathbf{G}_{i}\right| m_{i}, \quad m_{\text {out }}=g_{\text {out }} m_{\text {out }}^{\prime}
$$

is distinct from the rays generated by $-m_{i}$. Let $X^{o}$ be the corresponding open toric surface with divisors $D_{1}^{o}, \ldots, D_{n}^{o}, D_{\text {out }}^{o}$.

Select a distinct point $x_{i j}^{r}$ of $D_{i}^{o}$ for each part of $p_{i j}^{r}$ of $\mathbf{P}_{i}^{r}$. Let

$$
\nu: \widetilde{X}[\mathbf{G}] \rightarrow \widetilde{X}
$$


be obtained from $\widetilde{X}$ by taking $r$-orbifold blow-ups along $\left(x_{i j}^{r}, D_{i}^{o}\right)$ for all $i, r, j$. Let

$$
X^{o}[\mathbf{G}]=\nu^{-1}\left(X^{o}\right)
$$

Let $E_{i j}^{r} \subseteq \tilde{X}[\mathbf{G}]$ be the exceptional divisor over $x_{i j}^{r}$. Let $\beta \in H_{2}(\widetilde{X}, \mathbb{Z})$ be determined by intersection numbers with toric divisors,

$$
\widetilde{D}_{i} \cdot \beta=\left|\mathbf{G}_{i}\right|, \quad \widetilde{D}_{\text {out }} \cdot \beta=g_{\text {out }}
$$

and $D \cdot \beta=0$ if $D \notin\left\{\widetilde{D}_{1}, \ldots, \widetilde{D}_{n}, \widetilde{D}_{\text {out }}\right\}$. Let

$$
\beta_{\mathbf{G}}=\nu^{*}(\beta)-\sum_{i=1}^{n} \sum_{r=1}^{d_{i}} \sum_{j=1}^{\ell_{i}^{r}} p_{i j}^{r}\left[E_{i j}^{r}\right] \in H_{2}(\tilde{X}[\mathbf{G}], \mathbb{Z}) .
$$

Let $\overline{\mathfrak{M}}\left(\widetilde{X}[\mathbf{G}] / \widetilde{D}_{\text {out }}\right)$ be the moduli of stable relative maps of genus 0 curves representing the class $\beta_{\mathbf{G}}$ with full tangency of order $g_{\text {out }}$ at an unspecified point of $\widetilde{D}_{\text {out }}$. The moduli space of stable maps to orbifold targets is defined in [1, 4]. Since the relative divisor $\widetilde{D}_{\text {out }}$ does not meet the orbifold points of $\tilde{X}[\mathbf{G}]$, there is no difficulty in defining the moduli space of relative maps 13 Let

$$
\overline{\mathfrak{M}}\left(X^{o}[\mathbf{G}] / D_{\text {out }}^{o}\right) \subseteq \overline{\mathfrak{M}}\left(\widetilde{X}[\mathbf{G}] / \widetilde{D}_{\text {out }}\right)
$$

be the open subspace of maps which avoids $\widetilde{X}[\mathbf{G}] \backslash X^{o}[\mathbf{G}]$.

Since the curve class $\beta_{\mathbf{G}}$ accounts for all the intersections of the image of the map in $\widetilde{X}$, the argument for properness is still valid.

Proposition 5.5. $\overline{\mathfrak{M}}\left(X^{o}[\mathbf{G}] / D_{\text {out }}^{o}\right)$ is proper over $\operatorname{Spec}(\mathbb{C})$.

The virtual dimension of $\overline{\mathfrak{M}}\left(X^{o}[\mathbf{G}] / D_{\text {out }}^{o}\right)$ is 0 . Define the orbifold Gromov-Witten invariant as before

$$
N_{\mathbf{m}}[\mathbf{G}]=\int_{\left[\overline{\mathfrak{M}}\left(X^{o}[\mathbf{G}] / D_{\text {out }}^{o}\right)\right]^{v i r}} 1 .
$$

The integral $N_{\mathbf{m}}[\mathbf{G}]$ is independent of the locations of the distinct points $x_{i j}^{r} \in D_{i}^{o}$.

5.6. Full commutator formulas. Let $\mathfrak{d}_{1} \neq \mathfrak{d}_{2}$ be two lines through the origin in $M_{\mathbb{R}}$. Let $\mathbf{m}=\left(m_{1}, m_{2}\right)$ be the two associated primitive vectors. Let

$$
f_{\mathfrak{d}_{1}}=\prod_{r=1}^{d_{1}} \prod_{\xi=1}^{\ell_{1}^{r}}\left(1+s_{\xi}^{r} z^{r m_{1}}\right), \quad f_{\mathfrak{d}_{2}}=\prod_{r=1}^{d_{2}} \prod_{\xi=1}^{\ell_{2}^{r}}\left(1+t_{\xi}^{r} z^{r m_{2}}\right)
$$

over the complete ring $\mathbb{C}\left[\left[s_{\bullet}^{\bullet}, t_{\bullet}^{\bullet}\right]\right]$ in all the variables $s_{\xi}^{r}, t_{\xi}^{r}$.

\footnotetext{
${ }^{13}$ Orbifold stable maps are allowed also to have nontrivial prescribed orbifold structure on the domain. Our maps to $\tilde{X}[\mathbf{G}]$ have no such prescribed orbifold structure.
} 
For graded partitions $\mathbf{G}_{1}=\left(\mathbf{P}_{1}^{1}, \ldots, \mathbf{P}_{1}^{d_{1}}\right)$ and $\mathbf{G}_{2}=\left(\mathbf{P}_{2}^{1}, \ldots, \mathbf{P}_{2}^{d_{2}}\right)$, let

$$
s^{\mathbf{G}_{1}}=\prod_{r=1}^{d_{1}} \prod_{\xi=1}^{\ell_{1}^{r}}\left(s_{\xi}^{r}\right)^{\frac{p_{1 \xi}^{r}}{r}}, \quad t^{\mathbf{G}_{2}}=\prod_{r=1}^{d_{2}} \prod_{\xi=1}^{\ell_{2}^{r}}\left(t_{\xi}^{r}\right)^{\frac{p_{2 \xi}^{r}}{r}}
$$

be the corresponding monomials.

Again, we consider the unique minimal scattering diagram $\mathrm{S}(\mathfrak{D})$ associated to

$$
\mathfrak{D}=\left\{\left(\mathfrak{d}_{1}, f_{\mathfrak{d}_{1}}\right),\left(\mathfrak{d}_{2}, f_{\mathfrak{d}_{2}}\right)\right\} .
$$

Theorem 5.6. If $m_{\text {out }}^{\prime} \in \mathbb{Q}_{>0} m_{1}+\mathbb{Q}_{>0} m_{2}$,

$$
\log f_{m_{\text {out }}^{\prime}}=\sum_{k=1}^{\infty} \sum_{\mathbf{G}=\left(\mathbf{G}_{\mathbf{1}}, \mathbf{G}_{\mathbf{2}}\right)} k N_{\mathbf{m}}[\mathbf{G}] s^{\mathbf{G}_{1}} t^{\mathbf{G}_{2}} z^{k m_{\text {out }}^{\prime}}
$$

where the sum is over all graded partitions $\mathbf{G}_{1}$ of length $\left(\ell_{1}^{1}, \ldots, \ell_{1}^{d_{1}}\right)$ and $\mathbf{G}_{2}$ of length $\left(\ell_{2}^{1}, \ldots, \ell_{2}^{d_{2}}\right)$ satisfying

$$
\left|\mathbf{G}_{1}\right| m_{1}+\left|\mathbf{G}_{2}\right| m_{2}=k m_{\text {out }}^{\prime} .
$$

Proof. We follow the proof of Theorem 5.4. After factoring the diagram $\mathfrak{D}$ into $\sum_{r=1}^{d_{1}} \ell_{1}^{r}+\sum_{r=1}^{d_{2}} \ell_{2}^{r}$ lines, we will match the formula for $\log f_{m_{\text {out }}^{\prime}}$ from Theorem 2.8 with a degeneration calculation of the orbifold Gromov-Witten invariants of $X^{o}\left[\left(\mathbf{G}_{1}, \mathbf{G}_{2}\right)\right] / D_{\text {out }}^{o}$.

The geometric setting here is just as before. First, the degeneration

$$
\epsilon: \mathcal{F} \rightarrow \mathbb{C}
$$

defined in (5.2) is taken. The points $x_{1 \xi}^{r} \in D_{1}^{o}$ and $x_{2 \xi}^{r} \in D_{2}^{o}$ specialize to points of

$$
\mathbb{P}\left(\mathcal{O}_{D_{1}^{o}} \oplus \operatorname{Norm}_{X^{o} / D_{1}^{o}}\right) \text { and } \mathbb{P}\left(\mathcal{O}_{D_{2}^{o}} \oplus \operatorname{Norm}_{X^{o} / D_{2}^{o}}\right)
$$

respectively. The projective bundles (5.8) each contain two distinguished sections. The first section is the limit in $\mathcal{F}$ of the divisor $D_{i}^{o}$ and carries the limits of the $x_{i \xi}^{r}$. The second section meets $X^{o}$. There is no difficulty in taking the $r$-orbifold blow-ups relative to $\epsilon$. The underlying coarse space is obtained by blowing-up the families of canonically defined nilpotent subschemes, and the stack structure is uniquely determined. The resulting family,

$$
\epsilon_{\mathbf{G}}: \mathcal{F}[\mathbf{G}] \rightarrow \mathbb{C}
$$

has orbifold structure in the total space $\mathcal{F}[\mathbf{G}]$ with support disjoint from the relative divisors in the special fiber $\epsilon_{\mathbf{G}}^{-1}(0)$. Therefore, the usual degeneration formulas in relative Gromov-Witten theory hold unchanged.

A crucial fact used in Theorem 5.4 is the surprising match between the $a$ coefficients and the multiple cover contributions $R_{d}$ of Proposition 5.2. We need to be even luckier 
now. Since

$$
\begin{aligned}
& \log \left(1+s_{\xi}^{r} z^{r m_{1}}\right)=\sum_{d=1}^{\infty} d r \frac{(-1)^{d-1}}{r d^{2}} z^{d r m_{1}}\left(s_{\xi}^{r}\right)^{d}, \\
& \log \left(1+t_{\xi}^{r} z^{r m_{2}}\right)=\sum_{d=1}^{\infty} d r \frac{(-1)^{d-1}}{r d^{2}} z^{d r m_{2}}\left(t_{\xi}^{r}\right)^{d},
\end{aligned}
$$

the $a$ coefficients in both cases are

$$
a_{\xi, d, r d}^{r}=\frac{(-1)^{d-1}}{r d^{2}}
$$

These exactly match the orbifold multiple cover calculation in Proposition 5.7 below.

The remaining steps are identical to those taken in the proof of Theorem 5.4. The formula of Theorem 2.8 matches exactly the degeneration computation.

Let $P$ be the fiber of the projective bundle

$$
\mathbb{P}\left(\mathcal{O}_{D_{i}^{o}} \oplus \operatorname{Norm}_{X^{o} / D_{i}^{o}}\right) \rightarrow D_{i}^{o}
$$

containing the limit $\bar{x}_{i \xi}^{r}$. Let $C_{i \xi}^{r}$ be the strict transform of $P$ after taking the $r$-orbifold blow-up along $\left(\bar{x}_{i \xi}^{r}, D_{i}^{o}\right)$. Then,

$$
C_{i \xi}^{r} \stackrel{\sim}{=} \mathbb{P}^{1}[r, 1]
$$

with a single orbifold point of order $r$ at 0 . The normal bundle of $C_{i \xi}^{r}$ in the $r$-orbifold blow-up is simply $\mathcal{O}_{C_{i \xi}^{r}}\left(-[0] / \mathbb{Z}_{r}\right)$ of degree $-\frac{1}{r}$. For any map 14 from a genus 0 curve

$$
\varphi: C \rightarrow C_{i \xi}^{r}
$$

the degree of $\varphi$ must be a multiple of $r$. Let

$$
R_{d}^{r}=\int_{\left[\overline{\mathfrak{M}}\left(\mathbb{P}^{1}[r, 1] / \infty, r d\right)\right]^{v i r}} e\left(H^{1}\left(C, \varphi^{*}\left(\mathcal{O}_{C_{i \xi}^{r}}\left(-[0] / \mathbb{Z}_{r}\right)\right)\right)\right),
$$

where $\overline{\mathfrak{M}}\left(\mathbb{P}^{1}[r, 1] / \infty, r d\right)$ is the moduli space of genus 0 stable relative maps of degree $r d$ with full ramification $r d$ over $\infty$.

Proposition 5.7. $R_{d}^{r}=\frac{(-1)^{d-1}}{r d^{2}}$.

Proof. There is a $\mathbb{C}^{*}$ action on $\mathbb{P}^{1}[r, 1]$ with tangent weights $\left[\frac{1}{r},-1\right]$ at the fixed points $[0, \infty]$. The orbifold line bundle $\mathcal{O}_{C_{i \xi}^{r}}\left(-[0] / \mathbb{Z}_{r}\right)$ is canonically linearized with fiber weights $\left[-\frac{1}{r}, 0\right]$ over the respective fixed points.

We compute the integral $R_{d}^{r}$ via the induced $\mathbb{C}^{*}$ action on $\overline{\mathfrak{M}}\left(\mathbb{P}^{1}[r, 1] / \infty, r d\right)$ and the Bott residue formula. There are many $\mathbb{C}^{*}$-fixed loci in the moduli space. However, the

\footnotetext{
${ }^{14}$ We mean here a representable map. The domain $C$ has no stack structure.
} 
above linearization leads to the vanishing 15 of all contributions except for the single $\mathbb{C}^{*}$-fixed Galois cover

$$
\varphi: C \rightarrow \mathbb{P}^{1}[r, 1]
$$

of degree $r d$. The product of the weights of $\mathbb{C}^{*}$ on $H^{1}\left(C, \varphi^{*}\left(\mathcal{O}_{C_{i \xi}^{r}}\left(-[0] / \mathbb{Z}_{r}\right)\right)\right)$ are

$$
\prod_{i=1}^{d-1}-\frac{i}{r d}
$$

The product of the weights of $\mathbb{C}^{*}$ on the tangent space to $[\varphi]$ in $\overline{\mathfrak{M}}\left(\mathbb{P}^{1}[r, 1] / \infty, r d\right)$ is

$$
\frac{\prod_{i=1}^{d} \frac{i}{r d}}{\frac{1}{r d}}
$$

where the bottom factor is obtained from reparameterization over $0 \in \mathbb{P}^{1}[r, 1]$. The above weight calculations, obtained from the $\mathbb{C}^{*}$-equivariant geometry of basic orbifold line bundle on $\mathbb{P}^{1}[r, 1]$, are standard. See Section 2.2 of [12] for a detailed treatment.

By the Bott residue formula, $R_{d}^{r}$ equals the ratio of the above weights together with a stack automorphism factor of $\frac{1}{r d}$,

$$
R_{d}^{r}=\frac{1}{r d} \frac{\prod_{i=1}^{d-1}-\frac{i}{r d}}{\frac{\prod_{i=1}^{d} \frac{i}{r d}}{\frac{1}{r d}}}=\frac{(-1)^{d-1}}{r d^{2}}
$$

Our calculation is just a minor modification of Theorem 5.1 of [2].

Consider the tropical vertex group over the ring $\mathbb{C}[[t]]$. A function

$$
f=1+t z^{m} \cdot g\left(z^{m}, t\right) \quad g \in \mathbb{C}\left[z^{m}\right][[t]]
$$

attached to the ray with primitive $m$ can always be factored as

$$
f=\left(1+s_{1}^{1} z^{m}\right)\left(1+s_{1}^{2} z^{2 m}\right)\left(1+s_{1}^{3} z^{3 m}\right) \ldots
$$

for $s_{1}^{r} \in t^{n_{r}} \mathbb{C}[[t]]$ with

$$
\lim _{r \rightarrow \infty} n_{r}=\infty
$$

To any finite order in $t$, a suitable finite truncation of the factorization (5.9) suffices for any calculation. Therefore, we view Theorem 5.6 as an ordered product formula for an arbitrary commutator in the tropical vertex group.

We have written Theorem 5.6 for functions (5.7) in slightly more complicated form than (5.9) to capture all of the possible Gromov-Witten invariants which arise.

\footnotetext{
${ }^{15}$ See the proof of Theorem 5.1 in 2 where the same vanishing is explained.
} 
5.7. Ordered product formulas. Let $\mathfrak{D}$ be a scattering diagram with $n$ lines through the origin,

$$
\mathfrak{D}=\left\{\left(\mathfrak{d}_{i}, f_{i}\right) \mid 1 \leq i \leq n\right\} .
$$

Let $S(\mathfrak{D})$ be the unique minimal scattering diagram obtained by adding rays to $\mathfrak{D}$ for which the path ordered product around the origin is trivial. Let $m_{\text {out }}^{\prime} \in M$ be a primitive vector. The function $f_{m_{\text {out }}^{\prime}}$ is determined by the method used to prove Theorems 5.4 and 5.6 .

Let $m_{1}, \ldots, m_{n}$ be the primitives corresponding to the lines of $\mathfrak{D}$. Either $m_{\text {out }}^{\prime}$ is distinct from the $m_{i}$ or $m_{\text {out }}^{\prime}=m_{k}$ for some $k$. Consider the $n=3$ case with functions

$$
f_{1}\left(s_{\bullet}^{\bullet}\right), f_{2}\left(t_{\bullet}^{\bullet}\right), f_{3}\left(u_{\bullet}^{\bullet}\right)
$$

of the form (5.7). If $m_{\text {out }}^{\prime}$ is distinct, then

$$
\log f_{m_{\text {out }}^{\prime}}=\sum_{k=1}^{\infty} \sum_{\mathbf{G}=\left(\mathbf{G}_{\mathbf{1}}, \mathbf{G}_{\mathbf{2}}, \mathbf{G}_{\mathbf{3}}\right)} k N_{\mathbf{m}}[\mathbf{G}] s^{\mathbf{G}_{1}} t^{\mathbf{G}_{2}} u^{\mathbf{G}_{3}} z^{k m_{\text {out }}^{\prime}}
$$

where the sum is over all graded partitions $\mathbf{G}_{i}$ of lengths $\left(\ell_{i}^{1}, \ldots, \ell_{i}^{d_{i}}\right)$ satisfying

$$
\left|\mathbf{G}_{1}\right| m_{1}+\left|\mathbf{G}_{2}\right| m_{2}+\left|\mathbf{G}_{3}\right| m_{3}=k m_{\text {out }}^{\prime} .
$$

The same result holds for all $n$.

For the degenerate case $m_{\text {out }}^{\prime}=m_{k}$, the definition of the invariant $N_{\mathbf{m}}[\mathbf{G}]$ must be changed slightly. The only difference is the outgoing contact point $Q$ is placed on the divisor $D_{k}^{o}$ instead of $D_{\text {out }}^{o}$ (as discussed in Section 3.3). Then, equation (5.10) holds as written. We leave the straightforward details in the degenerate case to reader 16

5.8. Higher genus. The higher genus analogues of the genus 0 invariants $N_{\mathbf{m}}[\mathbf{G}]$ are not hard to construct. Let $\overline{\mathfrak{M}}_{g}\left(X^{o}[\mathbf{G}] / D_{\text {out }}^{o}\right)$ be the moduli space of genus $g$ stable relative maps representing the class $\beta_{\mathbf{G}}$ defined as in the genus 0 case. There are now two difficulties:

(i) $\overline{\mathfrak{M}}_{g}\left(X^{o}[\mathbf{G}] / D_{\text {out }}^{o}\right)$ is not proper,

(ii) $\overline{\mathfrak{M}}_{g}\left(X^{o}[\mathbf{G}] / D_{\text {out }}^{o}\right)$ is of virtual dimension $g$.

The issues are resolved simultaneously by defining

$$
N_{\mathbf{m}}^{g}[\mathbf{G}]=\int_{\left[\overline{\mathfrak{M}}_{g}\left(X^{o}[\mathbf{G}] / D_{\text {out }}^{o}\right)\right]^{v i r}}(-1)^{g} \lambda_{g}
$$

where $\lambda_{g}$ is the top Chern class of the Hodge bundle 17 Limits out of the moduli space $\overline{\mathfrak{M}}_{g}\left(X^{o}[\mathbf{G}] / D_{\text {out }}^{o}\right)$ lead to loops in the domain curve by the proof of Proposition 4.2 .

\footnotetext{
${ }^{16}$ For the commutator formulas of Theorem 5.4 and 5.6, nontrivial degenerate cases do not appear. The full arguments have been given there.

${ }^{17}$ See [18] for a parallel definition of higher genus invariants in the case of $K 3$ surfaces.
} 
However, the class $\lambda_{g}$ vanishes on the locus of curves with loops. Hence, $N_{\mathbf{m}}^{g}[\mathbf{G}]$ is

well-defined. A very interesting question is whether the relative invariants $N_{\mathbf{m}}^{g}[\mathbf{G}]$ can be related to the tropical vertex group.

\section{BPS STATE COUNTS}

6.1. Log Calabi-Yau. Let $S$ be a nonsingular surface and let $D \subseteq S$ be a nonsingular divisor. The pair $(S, D)$ is $\log C a l a b i$-Yau with respect to $0 \neq \beta \in H_{2}(S, \mathbb{Z})$ if

$$
D \cdot \beta=c_{1}(S) \cdot \beta \text {. }
$$

Two basic examples are:

- $\left(X^{o}[\mathbf{P}], D_{\text {out }}^{o}\right)$, constructed in Section 5.1, is log Calabi-Yau with respect to the class $\beta_{\mathbf{P}}$.

- $\left(\mathbb{P}^{2}, E\right)$, where $E$ is a nonsingular cubic, is log Calabi-Yau with respect to every class $\beta \in H_{2}\left(\mathbb{P}^{2}, \mathbb{Z}\right)$.

The moduli space $\overline{\mathfrak{M}}(S / D, w)$ of genus 0 stable relative maps to $S / D$ of class $\beta$ and full tangency of order $w=D \cdot \beta$ at a single unspecified point of $D$ is of virtual dimension 0 . Let

$$
N_{S}[w] \in \mathbb{Q}
$$

be the associated relative Gromov-Witten invariant. If

$$
\iota: P \rightarrow S
$$

is a rigid element of $\overline{\mathfrak{M}}(S / D, w)$, we can ask what is the contribution of $d$-fold multiple covers of $P$ to the Gromov-Witten invariant $N_{S}[d w]$ in class $d \beta$ ?

6.2. Multiple cover contributions. We pursue here multiple cover calculations and BPS state count definitions following the perspective of [21, 22]. In particular, we assume the map $\iota$ of (6.1) is as well-behaved as possible.

Let $\infty \in P$ be the point of contact with $D$. Let

$$
\overline{\mathfrak{M}}(P / \infty, d)^{*} \subseteq \overline{\mathfrak{M}}(S / D, d w)
$$

be the locus of genus 0 stable relative maps

$$
(C, Q) \rightarrow P / \infty \rightarrow S / D
$$

which factor as $d$-fold covers of $P$. The moduli space $\overline{\mathfrak{M}}(P / \infty, d)^{*}$ is a nonsingular Deligne-Mumford stack of dimension $d-1$. The superscript $*$ is used since the locus differs slightly from the standard moduli space of stable relative maps $\overline{\mathfrak{M}}(P / \infty, d)$. The reason is the $w$-tangency of $P$ with $D$ forces the ramification orders of maps over the destabilizations to all be divisible by $w$. We leave the details here for the reader. 
The contribution $M_{P}[d]$ of $d$-fold multiple covers of $P$ to $N_{S}[d w]$ is defined by

$$
M_{P}[d]=\int_{\left[\overline{\mathfrak{M}}(P / \infty, d)^{*}\right]} e\left(B_{d}\right)
$$

where $B_{d}$ is the obstruction bundle of rank $d-1$. On the open locus of $\overline{\mathfrak{M}}(P / \infty, d)^{\text {* }}$ consisting of maps

$$
\varphi:(C, Q) \rightarrow P / \infty
$$

with no destabilizations of the target, the obstruction space is

$$
H^{1}\left(C, \varphi^{*}\left(\operatorname{Norm}_{S / P}\right)(-(d w-d) Q)\right) .
$$

By adjunction, the degree of the normal bundle $\operatorname{Norm}_{S / P}$ is $w-2$. Hence, the degree of $\varphi^{*}\left(\operatorname{Norm}_{S / P}\right)(-(d w-d) Q)$ is $-d$ and the obstruction space (6.2) has rank $d-1$. A description of the obstruction space for relative maps can be found in [9].

Proposition 6.1. We have

$$
M_{P}[d]=\frac{1}{d^{2}}\left(\begin{array}{c}
d(w-1)-1 \\
d-1
\end{array}\right) .
$$

If $w=1$, then $\left(\begin{array}{c}-1 \\ d-1\end{array}\right)=(-1)^{d-1}$ by definition and the contribution

$$
M_{P}[d]=\frac{(-1)^{d-1}}{d^{2}}
$$

specializes to the genus 0 cap of [2].

Proof. The $\mathbb{C}^{*}$-action on $P$ fixing $\infty$ lifts to a $\mathbb{C}^{*}$-action on the moduli space $\overline{\mathfrak{M}}(P / \infty, d)^{*}$. Once the lifting of $\mathbb{C}^{*}$ to $\operatorname{Norm}_{S / P}$ is chosen, a lifting of $\mathbb{C}^{*}$ to the obstruction bundle $B_{d}$ is determined by the characterization of the obstruction space [9]. Let $[1,-1]$ be the tangent weights of $\mathbb{C}^{*}$ at the fixed points $0, \infty \in P$. We chose a lifting of $\mathbb{C}^{*}$ to Norm $_{S / P}$ by specifying fiber weights $[w-2,0]$ over the respective fixed points.

We compute the integral $M_{P}[d]$ via the Bott residue formula. There are many $\mathbb{C}^{*}$ fixed loci in the moduli space $\overline{\mathfrak{M}}(P / \infty, d)^{*}$. However, the above linearization leads to the vanishing of all contributions except for the single $\mathbb{C}^{*}$-fixed Galois cover

$$
\varphi:(C, Q) \rightarrow P / \infty
$$

of degree $d$. If $w=1$, the vanishing is the same as in the proof of Theorem 5.1 of [2].

If $w>1$, a different argument is needed. Consider a $\mathbb{C}^{*}$-fixed locus of

$$
\mathfrak{L} \subseteq \overline{\mathfrak{M}}(P / \infty, d)^{*}
$$

for which the target is destabilized. For

$$
[\varphi:(C, Q) \rightarrow P / \infty] \in \mathfrak{L},
$$


let $C^{\prime}, C^{\prime \prime} \subseteq C$ be the subcurves mapped by $\varphi$ to the original and destabilizing components of the target respectively. The $\mathbb{C}^{*}$-action on the pull-back to $C^{\prime}$ of $\operatorname{Norm}_{S / P}$ is nontrivial. However, since $w>1$,

$$
H^{1}\left(C^{\prime}, \varphi^{*}\left(\operatorname{Norm}_{S / P}\right)\right)=0 .
$$

The $\mathbb{C}^{*}$-action on the pull-back to $C^{\prime \prime}$ of $\operatorname{Norm}_{S / P}$ is trivial by our choice of lifting. The $\mathbb{C}^{*}$-action on the destabilizing components of the target is trivial. The point $Q$ must map to a destabilizing component of the target, hence the $\mathbb{C}^{*}$-action on $\mathcal{O}_{C}(-Q)$ is trivial. By examining the obstruction space [9], we conclude the $\mathbb{C}^{*}$-action on $B_{d}$ is trivial over $\mathfrak{L}$. By dimension considerations, the contribution of $\mathfrak{L}$ vanishes in the Bott residue formula - specifically the Euler class $e\left(B_{d}\right)$ is 0 when restricted to $\mathfrak{L}$. The Galois cover (6.3) is the unique $\mathbb{C}^{*}$-fixed locus for which the target is not destabilized.

We compute the contribution of the Galois cover to the Bott residue formula. The weights of $\mathbb{C}^{*}$ on the fibers of $\varphi^{*}\left(\operatorname{Norm}_{S / P}\right)(-(d w-d) Q)$ over the respective fixed points on $C$ are $[w-2, w-1]$. The weights of $\mathbb{C}^{*}$ on $H^{1}\left(C, \varphi^{*}\left(\operatorname{Norm}_{S / P}\right)(-(d w-d) Q)\right)$ are

$$
\prod_{i=1}^{d-1} \frac{d w-d-i}{d}
$$

The weights of $\mathbb{C}^{*}$ on the tangent space to $[\varphi]$ in $\overline{\mathfrak{M}}(P / \infty, d)^{*}$ are

$$
\frac{\prod_{i=1}^{d} \frac{i}{d}}{\frac{1}{d}}
$$

where the bottom factor is obtained from reparameterization over $0 \in P$. By the Bott residue formula, $M_{P}[d]$ equals the ratio of the above weights,

$$
M_{P}[d]=\frac{1}{d} \frac{\prod_{i=1}^{d-1} \frac{d w-d-i}{d}}{\frac{\prod_{i=1}^{d} \frac{i}{d}}{\frac{1}{d}}}=\frac{1}{d^{2}}\left(\begin{array}{c}
d(w-1)-1 \\
d-1
\end{array}\right),
$$

together with a stack automorphism factor of $\frac{1}{d}$.

6.3. Conjectures. Let $(S, D)$ be a log Calabi-Yau pair with respect to a primitive class $\beta \neq 0$. Let $w=D \cdot \beta$ as before. Consider the generating series

$$
N_{S}=\sum_{k=1}^{\infty} N_{S}[k w] q^{k}
$$

Using the multiple cover calculation, we can write

$$
N_{S}=\sum_{k=1}^{\infty} n_{S}[k w] \sum_{d=1}^{\infty} \frac{1}{d^{2}}\left(\begin{array}{c}
d(k w-1)-1 \\
d-1
\end{array}\right) q^{d k}
$$

for unique numbers $n_{S}[k w] \in \mathbb{Q}$. Equation (6.4) defines the $n_{S}[k w]$. 
Conjecture 6.2. The $n_{S}[k w]$ are integers for all $k \geq 1$.

Extracting integers from genus 0 Gromov-Witten theory by removing multiple cover contributions is a basic idea in the subject - first pursued in the study of genus 0 curves on the quintic 3-fold. By the string theoretic work of Gopakumar and Vafa [7, 8], the resulting integers can often be interpreted as BPS state counts in related theories. We interpret $n_{S}[k w]$ here as the associated BPS count. Unfortunately, in almost every case, integrality statements of the form of Conjecture 6.2 are not provable by existing techniques 18

Conjecture 6.2 applies to the geometries $\left(X^{o}[\mathbf{P}], D_{\text {out }}^{o}\right)$ associated to the functions $f_{m_{\text {out }}^{\prime}}$ in Theorem 5.4. Kontsevich and Soibelman conjectured [14] an equivalent integrality 19 for the functions $f_{m_{\text {out }}^{\prime}}$ associated to such commutators, and a proof via quiver techniques has been recently provided in [24]. Conjecture 6.2 applies to other quite different situations as well. The most interesting case is perhaps the log Calabi-Yau pair $\left(\mathbb{P}^{2}, E\right)$ studied in [5, 25]. In all the examples we have considered, there is good numerical evidence supporting Conjecture 6.2.

Conjecture 6.2 does not apply as stated to the orbifold geometries $\left(X^{o}[\mathbf{G}] / D_{\text {out }}^{o}\right)$ of Theorem 5.6. The orbifold structure leads to more complicated multiple cover contributions which we have not yet calculated.

6.4. Examples. Consider the $\ell_{1}=\ell_{2}=3$ case of the commutator (0.2) of the Introduction. The corresponding scattering diagram is discussed in Example 1.6 of Section 1.3. Focus on the function attached to the line of slope 1. By direct calculation in the tropical vertex group, we find

$$
\begin{aligned}
\log f_{\text {out }}=9\left(t_{1} t_{2} x y\right)+2 \cdot \frac{63}{4}\left(t_{1} t_{2} x y\right)^{2} & +3 \cdot 55\left(t_{1} t_{2} x y\right)^{3} \\
& +4 \cdot \frac{4095}{16}\left(t_{1} t_{2} x y\right)^{4}+5 \cdot \frac{100947}{25}\left(t_{1} t_{2} x y\right)^{5}+\cdots
\end{aligned}
$$

Consider $\mathbb{P}^{2}$ with the coordinate axes $D_{1}, D_{2}$ and $D_{\text {out }}$ and pick points

$$
x_{11}, x_{12}, x_{13} \in D_{1}, \quad x_{21}, x_{22}, x_{23} \in D_{2}
$$

\footnotetext{
${ }^{18}$ One exception is the Fano 3-fold case settled in [26]. Calabi-Yau cases such as ours here are more difficult.

${ }^{19}$ This conjecture, in our language, would posit a multiple cover contribution of the form $(-1)^{d-1} / d^{2}$ independent of $w$. As we have seen, the multiple cover contributions are more subtle (and depend upon $w)$. But, the associated integrality is the same.
} 
Sums of the relative Gromov-Witten invariants $N_{\mathbf{m}}\left[\left(\mathbf{P}_{1}, \mathbf{P}_{2}\right)\right]$ are determined by Theorem 5.4. For example,

$$
\sum_{\left|\mathbf{P}_{1}\right|=1,\left|\mathbf{P}_{2}\right|=1} N_{\mathbf{m}}\left[\left(\mathbf{P}_{1}, \mathbf{P}_{2}\right)\right]=9 .
$$

We can easily interpret the answer in the following way. Given a choice of one of the three points on $D_{1}$ and one of the three points on $D_{2}$, there is precisely one line through these two points, which of course is maximally tangent to $D_{\text {out }}$. There are nine such configurations, hence the correct answer is 9 .

The next coefficient of $f_{\text {out }}$ yields

$$
\sum_{\left|\mathbf{P}_{1}\right|=2,\left|\mathbf{P}_{2}\right|=2} N_{\mathbf{m}}\left[\left(\mathbf{P}_{1}, \mathbf{P}_{2}\right)\right]=\frac{63}{4} .
$$

The double covers of the lines mentioned above count for $-9 / 4$, leaving a contribution of $63 / 4+9 / 4=18$ from conics passing through two of the three points on $D_{1}$ and two of the three points on $D_{2}$. Indeed, given any choice of two points each on $D_{1}$ and $D_{2}$, there are two conics through these four points tangent to $D_{\text {out }}$.

The third coefficient is more interesting. We have

$$
\sum_{\left|\mathbf{P}_{1}\right|=3,\left|\mathbf{P}_{2}\right|=3} N_{\mathbf{m}}\left[\left(\mathbf{P}_{1}, \mathbf{P}_{2}\right)\right]=55 .
$$

The contribution from triple covers of the lines is $9 / 3^{2}=1$, hence we expect 54 nonmultiply covered cubics. A refined scattering diagram calculation reveals more specifically the following numbers:

$$
\begin{aligned}
& N_{\mathbf{m}}[1+1+1,1+1+1]=18 \\
& N_{\mathbf{m}}[2+1+0,1+1+1]=3
\end{aligned}
$$

The first number can be interpreted as the number of nodal cubics passing through all six of the chosen points and maximally tangent to $D_{\text {out }}$, while the second number can be interpreted as the number of nodal cubics passing through $x_{11}$ twice (so that the node is at $x_{11}$ ), passing through $x_{12}, x_{21}, x_{22}$ and $x_{23}$ once, and again maximally tangent to $D_{\text {out }}$. There are a total of 12 partitions $\mathbf{P}=\left(\mathbf{P}_{1}, \mathbf{P}_{2}\right)$ involving the numbers $2,1,0,1,1,1$ of this sort, so the total accounting is

$$
54=18+12 \times 3
$$

In the above cases, the Gromov-Witten invariants (corrected for multiple covers) solve straight counting problems. 


\section{Appendix: Tropical/holomorphic COUnts}

The purpose of this Appendix is to discuss the modifications of [20] necessary to obtain a proof of Theorem 3.4. We do not try to be self-contained and rather just indicate what has to be changed.

First, some conventions of [20] conflict with the notation adopted in the present paper. Most importantly, the roles of $M$ and $N$ are reversed. The reason is that fans and tropical curves traditionally live in $N$, but the tropical vertex group naturally acts on polyomial rings with exponents on the dual lattice $M$. Moreover, $n$ denotes the rank of $N$ in [20], while now we work in dimension 2 and $n$ denotes the number of incoming directions. In [20], our toric variety $X$ is written $X(\Sigma)$ and $X$ denotes the total space of a toric degeneration. Another irrelevant difference is that in [20] we do tropical geometry over $\mathbb{Q}$ while here we work over $\mathbb{R}$. In the Appendix, we follow the notation of [20] except for swapping $N$ and $M$.

The degree of the tropical curves to be considered is fixed by the number and directions of the incomming edges. Rather than imposing incidence of a marked edge with an affine subspace as in Definition 1.3 of [20], we constrain the incoming unbounded edge $E_{i j}$ by the choice of an element $m_{i j} \in M_{\mathbb{Q}} / \mathbb{Q} m_{i}$. The latter is equivalent to the condition

$$
h\left(E_{i j}\right) \subseteq \mathfrak{d}_{i j}=m_{i j}+\mathbb{Q} m_{i}
$$

of Definition 2.6. The tuple $\mathbf{A}=\left(m_{i j}\right)$ with $m_{i j} \in M_{\mathbb{Q}} / \mathbb{Q} m_{i}$ determines a constraint. A marked tropical curve $\left(\Gamma,\left(E_{i j}\right), h\right)$ matches the constraint $\mathbf{A}$ if for all $i, j$

$$
h\left(E_{i j}\right)=\left\{m_{i j}\right\} \quad \text { in } M_{\mathbb{Q}} / \mathbb{Q} m_{i} .
$$

The finiteness and transversality results in Section 2 of [20] carry over without difficulty, but are partly already contained in Theorem 2.4. The gluing map (4) in [20] now reads

$$
\begin{aligned}
\Phi: \operatorname{Map}\left(\Gamma^{[0]}, M_{\mathbb{Q}}\right) & \longrightarrow \prod_{E \in \Gamma^{[1]} \backslash \Gamma_{\infty}^{[1]}} M_{\mathbb{Q}} / \mathbb{Q} u_{\left(\partial^{-} E, E\right)} \times \prod_{i, j} M_{\mathbb{Q}} / \mathbb{Q} m_{i}, \\
h & \longmapsto\left(\left(h\left(\partial^{+} E\right)-h\left(\partial^{-} E\right)\right)_{E}, h\left(\partial^{-} E_{i j}\right)-m_{i j}\right) .
\end{aligned}
$$

In Section 3 of [20] we need to adapt the treatment of incidence points, which in the present situation lie on the toric boundary. Let

$$
\pi: X=X\left(\widetilde{\Sigma}_{\mathscr{P}}\right) \rightarrow \mathbb{A}^{1}
$$

be the toric degeneration defined by an integral polyhedral decomposition $\mathscr{P}$ of $M_{\mathbb{Q}}$, and let $X_{t}=\pi^{-1}(t)$. Let $D \subseteq X$ be the union of those toric divisors of $X$ not contained in $X_{0}$, that is, corresponding to rays of $\widetilde{\Sigma}_{\mathscr{P}}$ contained in $M_{\mathbb{Q}} \times\{0\}$. The intersection $D_{t}=D \cap X_{t}$ for $t \neq 0$ is the toric boundary of $X_{t}$, while $D_{0} \subseteq X_{0}$ is the union of 
those 1-codimensional toric strata not contained in the singular locus of $X_{0}$. We thus consider $D_{0}$ as the toric boundary of $X_{0}$.

The asymptotic fan $\Sigma_{\mathscr{P}}$ of $\mathscr{P}$ is the fan defining $X_{t}$ for any $t \neq 0$. Thus if $\omega=\mathbb{Q}_{\geq 0} \cdot u$ is a ray of $\Sigma_{\mathscr{P}}$, then $\omega \times\{0\}$ is a ray of $\widetilde{\Sigma}_{\mathscr{P}}$. The fan describing the associated toric divisor $\widetilde{D}_{\omega} \subseteq D$ consists of the images under the projection

$$
M_{\mathbb{Q}} \times \mathbb{Q} \rightarrow\left(M_{\mathbb{Q}} / \mathbb{Q} u\right) \times \mathbb{Q}
$$

of the cones $\sigma \in \widetilde{\Sigma}_{\mathscr{P}}$ containing $\omega \times\{0\}$. This is again a fan of cones over the cells of a polyhedral decomposition $\mathscr{P}_{\omega}$, now of $M_{\mathbb{Q}} / \mathbb{Q} u$. The associated toric degeneration is $\left.\pi\right|_{\tilde{D}_{\omega}}$. The vertices of $\mathscr{P}_{\omega}$, or equivalently the irreducible components of

$$
D_{\omega}:=\widetilde{D}_{\omega} \cap X_{0}
$$

are in bijective correspondence with the unbounded edges of $\mathscr{P}$ in direction $\omega$. If $\widetilde{\omega}$ is such an unbounded edge and $D_{\widetilde{\omega}} \subseteq D_{\omega}$ the corresponding irreducible component, the linear space generated by $\widetilde{\omega} \times\{1\}$ in $\left(M_{\mathbb{Q}} / \mathbb{Q} u\right) \times \mathbb{Q}$ defines a 1 -dimensional subtorus $\mathbb{G}_{\widetilde{\omega}}$ of $\mathbb{G}((M / \mathbb{Z} u) \times \mathbb{Z})$, the stabilizer of $D_{\widetilde{\omega}} \subseteq \widetilde{D}_{\omega}$. By Corollary 3.8 of [20], given a closed point $Q \in D_{t}$ for $t \neq 0$ and assuming $\mathscr{P}$ integral, the closure of the orbit $\mathbb{G}_{\tilde{\omega}} \cdot Q$ is a section $\widetilde{Q}$ of

$$
\widetilde{D}_{\omega} \rightarrow \mathbb{A}^{1}
$$

with $\widetilde{Q} \cap X_{0} \subseteq D_{\widetilde{\omega}}$. Summarizing, the choice of $l_{i}$ unbounded edges in direction $u=-m_{i}$ in a polyhedral decomposition with asymptotic fan $\Sigma$ readily defines a degeneration of our incidence points $Q_{i 1}, \ldots, Q_{i l_{i}}$ to points $Q_{i j}^{0} \in X_{0}$ on disjoint toric strata of $\widetilde{D}_{\omega_{i}}$, $\omega_{i}=-\mathbb{Q}_{\geq 0} m_{i}$, the degeneration of the toric divisor $D_{i} \subseteq X_{t}=X(\Sigma)$.

For a general constraint $\mathbf{A}$, the affine map (6.5) is an isomorphism. If $\mathfrak{D} \in \mathbb{N}$ is the index of the corresponding inclusion of lattices, Proposition 5.7 of [20] about the existence of exactly $\mathfrak{D}$ isomorphism classes of maximally degenerate curves matching the incidence conditions on $X_{0}$ works as before. The only difference is that the $i j$-th incidence condition is now a torsor under $\mathbb{G}\left(M / \mathbb{Z} m_{i}\right)$.

In the deformation theory of Section 7 of [20], the discussion of the situation at the toric boundary is somewhat hidden. In the notation of [20], the above discussion provides the degeneration of toric boundaries $\underline{D} \subseteq \underline{X}$. Now there are functions on $X$ vanishing along $\underline{D}$, but not on $\underline{X_{0}}$. These force the introduction of special (marked) points in the log-structure of $C_{0}$, and the order of tangency of $\underline{\varphi_{0}}$ with $\underline{D}$ at such a point $x \in \underline{C_{0}}$ is fixed by the induced map of ghost sheaves

$$
\underline{\varphi}_{0}^{*} \overline{\mathcal{M}}_{X} \longrightarrow \overline{\mathcal{M}}_{C_{0}}
$$

In fact, $\overline{\mathcal{M}}_{X, \varphi_{0}(x)}$ has a direct summand $\mathbb{N}$ generated by a local equation for $\underline{D}$, and similarly $\overline{\mathcal{M}}_{C_{0}, x}$ has a direct summand $\mathbb{N}$ coming from a local equation for the marked 
point. The map on these direct summands is multiplication with the order of tangency. Log deformation theory preserves this map and hence considers deformations with fixed tangency conditions from the outset. Thus there is nothing to be changed here.

The only difference is again the discussion of the incidence conditions via the transversality argument in Proposition 7.3 of [20]. In our situation, the point is to show surjectivity of the evaluation map

$$
H^{0}\left(\mathcal{N}_{\varphi_{0}}\right) \longrightarrow \prod_{i, j} T_{X / \mathbb{A}^{1}, \underline{\varphi_{0}}}\left(x_{i j}\right) / D \varphi_{0}\left(T_{C_{0} / O_{0}, x_{i j}}\right)
$$

just as in (9) of [20]. This is the same as the second component

$$
\operatorname{Map}\left(\Gamma^{[0]}, M\right) \longrightarrow \prod_{i, j} M / \mathbb{Q} m_{i}
$$

of (6.5), tensored with $\mathbb{C}$, so is surjective for tropical curves that are general in the sense of Definition 2.3 of [20].

Therefore, fixing A general, the arguments of Section 8 of [20] produce a bijective correspondence between holomorphic curves contributing to $N_{\mathbf{m}}^{\mathrm{hol}}(\mathbf{w})$ in $X_{t}$ for small $t$ and certain stable log-maps to $X_{0}$, for a fixed degeneration

$$
X \rightarrow \mathbb{A}^{1}
$$

Each stable log map yields a tropical curve contributing to $N_{\mathbf{m}}^{\text {trop }}(\mathbf{w})$, computed with the asymptotics provided by $\mathbf{A}$. Conversely, a tropical curve $(\Gamma, \mathbf{E}, h)$ has $\mathfrak{D}(\Gamma, \mathbf{E}, h) \cdot w(\Gamma)$ stable $\log$ maps associated to it. Here $\mathfrak{D}(\Gamma, \mathbf{E}, h)$ is the lattice index associated to (6.5), and $w(\Gamma)$ is the product of all weights of bounded edges. Thus $(\Gamma, \mathbf{E}, h)$ contributes

$$
w(\Gamma) \cdot \mathfrak{D}(\Gamma, \mathbf{E}, h)
$$

to $N_{\mathbf{m}}^{\text {hol }}(\mathbf{w})$. Now $\mathfrak{D}(\Gamma, \mathbf{E}, h)$ is the same as the lattice index $\mathfrak{D}(\Gamma, \mathbf{E}, h, \mathbf{P})$ of [20] for imposing pointwise incidence conditions $\mathbf{P}=\left(P_{i j}\right)$ on $h\left(E_{i j}\right)$. Proposition 8.8 of [20] thus implies

$$
w(\Gamma, \mathbf{E}) \cdot \mathfrak{D}(\Gamma, \mathbf{E}, h)=\prod_{V \in \Gamma^{[0]}} \operatorname{Mult}_{V}(h),
$$

where we used the notation of Definition 2.2 and $w(\Gamma, \mathbf{E})=w(\Gamma) \cdot \prod_{i, j} w_{i j}$ in the present case 20 Hence,

$$
N_{\mathbf{m}}^{\mathrm{hol}}(\mathbf{w})=\frac{1}{\prod_{i, j} w_{i j}} \sum_{\{(\Gamma, \mathbf{E}, h)\}} \prod_{V \in \Gamma^{[0]}} \operatorname{Mult}_{V}(h)=\frac{N_{\mathbf{m}}^{\mathrm{trop}}(\mathbf{w})}{\prod_{i, j} w_{i j}},
$$

finishing the proof of Theorem 3.4

\footnotetext{
${ }^{20}$ The statement of Proposition 8.8 of [20] with $w(\Gamma)$ rather than $w(\Gamma, \mathbf{E})$ is wrong, and in fact, $w(\Gamma, \mathbf{E})$ is also needed there for the claimed equivalence with the mutitplicity of [19]. The problem is an incorrect verification of the base case of the induction. The rest of the proof remains the same.
} 


\section{REFERENCES}

[1] D. Abramovich, T. Graber, A. Vistoli, Gromov-Witten theory for Deligne-Mumford stacks, math/0603151.

[2] J. Bryan and R. Pandharipande, Curves in Calabi-Yau threefolds and Topological Quantum Field Theory, Duke Math. J. 126 (2005), 369-396.

[3] J. Bryan and R. Pandharipande, Local Gromov-Witten theory of curves, J. Amer. Math. Soc. 21 (2008), 101-136.

[4] W. Chen and Y. Ruan, Orbifold Gromov-Witten theory in Orbifolds in mathematics and physics (Madison, WI, 2001), 25-85, Contemp. Math. 310 (2002).

[5] A. Gathmann, Relative Gromov-Witten invariants and the mirror formula, Math. Ann. 325 (2003), $393-412$.

[6] A. Gathmann and H. Markwig, The numbers of tropical plane curves through points in general position, math/0504390. Adv. in Math. 217 (2008), 537-560.

[7] R. Gopakumar and C. Vafa, M-theory and topological strings I, hep-th/9809187.

[8] R. Gopakumar and C. Vafa, M-theory and topological strings II, hep-th/9812127.

[9] T. Graber and R. Vakil, Relative virtual localization and vanishing of tautological classes on moduli spaces of curves, Duke Math. J. 30 (2005), 1-37.

[10] M. Gross and B. Siebert, From real affine geometry to complex geometry, math.AG/0703822.

[11] E. Ionel and T. Parker, Relative Gromov-Witten invariants, Ann. of Math. 157 (2003), 45-96.

[12] P. Johnson, R. Pandharipande, H.-H. Tseng, Abelian Hurwitz-Hodge integrals, arXiv:0803.2356.

[13] M. Kontsevich and Y. Soibelman, Affine structures and non-Archimedean analytic spaces, in: The unity of mathematics (P. Etingof, V. Retakh, I.M. Singer, eds.), 321-385, Progr. Math. 244, Birkhäuser 2006.

[14] M. Kontsevich and Y. Soibelman, Stability structures, motivic Donaldson-Thomas invariants and cluster transformations, arXiv:0811.2435.

[15] A.-M. Li and Y. Ruan, Symplectic surgery and Gromov-Witten invariants of Calabi-Yau 3-folds I, Invent. Math. 145 (2001), no. 1, 151-218.

[16] J. Li, Stable morphisms to singular schemes and relative stable morphisms, J. Diff. Geom. 57 (2001), 509-578.

[17] J. Li, A degeneration formula for Gromov-Witten invariants, J. Diff. Geom. 60 (2002), 199-293.

[18] D. Maulik and R. Pandharipande, Gromov-Witten theory and Noether-Lefschetz theory, arXiv:0705.1653.

[19] G. Mikhalkin, Enumerative tropical algebraic geometry in $\mathbb{R}^{2}$, J. Amer. Math. Soc. 18 (2005), 313-377.

[20] T. Nishinou and B. Siebert, Toric degenerations of toric varieties and tropical curves, Duke Math. J. 135 (2006), 1-51.

[21] R. Pandharipande, Hodge integrals and degenerate contributions, Comm. Math. Phys. 208 (1999), 489-506

[22] R. Pandharipande, Three questions in Gromov-Witten theory, Proceedings of the ICM (Beijing 2002), Vol II., 503-512. 
[23] M. Reineke, Poisson automorphisms and quiver moduli, arXiv:0804.3214.

[24] M. Reineke, Cohomology of quiver moduli, functional equations, and integrality of Donaldson-Thomas type invariants, arXiv:0903.0261

[25] N. Takahashi, Log mirror symmetry and local mirror symmetry, math.AG/0004179.

[26] A. Zinger, A comparison theorem for Gromov-Witten invariants in the symplectic category, arXiv:0807.0805.

Department of Mathematics, UCSD, La Jolla, CA 92093, USA

E-mail address: mgross@math.ucsd.edu

Department of Mathematics, Princeton Univerity, Princeton, NJ 08544, USA

E-mail address: rahulp@math.princeton.edu

Department Mathematik, Universität Hamburg, 20146 Hamburg, Germany

E-mail address: bernd.siebert@math.uni-hamburg.de 